\title{
Modeling and Analysis of Wireless Networks with Interference Management
}

\section{Zeinab Yazdanshenasan Shahraki}

School of Electrical and Electronic Engineering

A thesis submitted to Nanyang Technological University in partial fulfillment of the requirements for the degree of $\mathrm{PhD}$ 
Dedicated to my family. 


\section{Acknowledegment}

I would like to express my sincere gratitude to my supervisor Prof. Peter Chong from NTU and Prof. Harpreet Dhillon from VT for their precious support, guidance, understanding and supervising this research work. I am indebted to them for helping me find the right problem to focus as well as for ensuring me an appropriate work environment where I could develop my research activities and pursue my objects.

I would also like to thank Prof. Yong Liang Guan for his support during the last months of my research. In addition to my advisors, I would like to extend my gratitude to Dr. Sayandeev Mukherjee that his guidance was invaluable in getting me started with Stochastic geometry. I would never forget his sincere guidance. Also, I would like to thank Prof. Tony Quek for his suggestions and comments that contributed to improve this work.

My special thanks goes to Singapore International Graduate Award (SINGA) which provides financial support during my Ph.D. studies. I would like to thank all my Infinitus colleagues for maintaining a motivational work environment with their friendly and supportive attitude, especially Mehrnaz Afshang for her continuous support and kindness both on my research and in my life. 
My sincere appreciation also goes to Ramtin Rabiee and Shideh Madadi who always had the patience to listen to me and help me in every way possible.

Foremost, I would like to thank my husband for his patience, support, encouragement as well as his love and faith on my aspirations. Equally important, I thank my parents, for their undeniable supports. 


\section{Abstract}

Next generation wireless networks integrate wireless technologies to support massive data requirements and seamless connectivity. Since interference has been the main limiting factor ever since wireless communication evolved, characterizing interference field accurately is an important step toward the design and deployment of wireless networks. Stochastic geometry has emerged as an important tool for the analysis of wireless networks. Initially popular for the modeling of ad hoc and wireless sensor networks, stochastic geometry has recently been adopted for the analysis of cellular and heterogeneous cellular networks as well. In this dissertation, we develop tractable analytical frameworks for the modeling and analysis of interference management techniques in wireless networks by using tools from stochastic geometry.

In the context of random spatial models, this dissertation first presents mathematical preliminaries and fundamental tools for the network modeling based on stochastic geometry approaches. We provide an overview on point process models and different approaches used for the analysis of the network. In this work, we focus on the characterization of interference in a Poisson hole process (PHP) model. Interference field in wireless networks is often modeled by a homogeneous Poisson point process (PPP). While it is realistic in modeling the inherent node irregularity and provides meaningful first-order results, PPP falls short in modeling the effect of interference management techniques, which typically introduce some form of spatial interaction among active transmitters. In some applications, such as cognitive radio and deviceto-device networks, this interaction may result in the formation of holes that are areas with low interference field strength in an otherwise homogeneous interference field. The resulting interference field can be accurately modeled as a PHP. Despite the importance of PHP in many applications, the exact characterization of interference experienced by a typical node in a PHP is not known.

In this dissertation, we first derive several tight upper and lower bounds on the Laplace transform of this interference. Numerical comparisons reveal that the new bounds outperform all known bounds and approximations, and are remarkably tight in all operational regimes of interest. The key in deriving these tight and yet simple bounds is to capture the local neighborhood information around the typical node accurately while simplifying the far field to attain tractability. Ideas for tightening these bounds further by incorporating the effect of overlaps in the holes are also discussed. These results immedi- 
ately lead to an accurate characterization of the coverage probability of the typical node in a PHP under Rayleigh fading.

Second, we develop our proposed approach to study the coverage in a PHP-based heterogeneous cellular network model with dependence. Actual cellular networks reveal spatial separation among base station (BS) deployments belonging to different tiers. While PPP is highly desired for signal-tointerference-plus-noise ratio (SINR) characterization in heterogeneous cellular networks (HCNs) due to its analytical tractability and accuracy, ignoring the spatial correlation of the BSs from different tiers appears unrealistic. We propose a new approach for the analysis of a two-tier HCN when the small cell BSs form a PHP. This model which is recently used for the modeling and analysis of HCNs guarantees a minimum distance between BSs of different tiers while induces spatial correlations among them. We develop the analytical framework that is proposed to capture holes in an ad-hoc network by an equivalent non-homogeneous PPP for the analysis of a $\mathrm{HCN}$. We provide a framework which focuses on a closed access HCN that can be extended to open access strategy.

Our third contribution is to focus on the characterization of asymmetric interference field. The asymmetric exclusion zones can be formed by interference management techniques in wireless communication networks. One of the realistic scenarios in this context appears when an exclusion zone is established to protect the communication of the typical user located at an arbitrary location inside the cell, this is unlike most existing works which assume that the symmetric exclusion zones protect the typical user located at the center of the cell. We propose an approach to capture this asymmetric interference field by an equivalent nonhomogeneous PPP. This transformation is also applied for a finite network which is more complicated in modeling aggregate interference field, and is more challenging in evaluating the performance of the system. A general framework is provided by the approach to facilitate the analysis and characterize the network performance accurately. 


\section{Table of Contents}

Dedication ..................... I I

Acknowledegment . . . . . . . . . . . . . . . II

Abstract . . . . . . . . . . . . . . IV

Table of Contents . . . . . . . . . . . . . . VI

List of Figures . . . . . . . . . . . . . . . . . . IX

List of Tables . . . . . . . . . . . . . . . . XI

List of Acronyms . . . . . . . . . . . . . . . XII

1 Introduction $\quad 1$

1.1 Interference Management Techniques in Wireless Networks . . 6

1.2 Related Works and Motivation . . . . . . . . . . . . . . . . 10

1.3 Contributions and Outcomes . . . . . . . . . . . . . . . 12

1.4 Organization of the Thesis . . . . . . . . . . . . . . . 15

2 Overview of Network Modeling $\quad 17$

2.1 Point Process Models . . . . . . . . . . . . . . . . . . . . . . . 19

2.1.1 Poisson Point Processes _. . . . . . . . . . . . 20

2.1.2 Hard-core Processes . . . . . . . . . . . . . . . . . . . 21

2.1.3 Cluster Processes . . . . . . . . . . . . . . . . 22

2.1.4 Cox Processes . . . . . . . . . . . . . . . . . . 22

2.2 Network Performance Analysis . . . . . . . . . . . . . . . . . . 23

2.3 Stochastic Geometry Modeling for Tiered Networks . . . . . . 25

3 Poisson Hole Process: Theory and Applications to Wireless $\begin{array}{ll}\text { Networks } & 28\end{array}$

3.1 Introduction . . . . . . . . . . . . . . . . . . . . . 28

3.1.1 Related Work and Applications . . . . . . . . . . 30 
3.2 Network Model . . . . . . . . . . . . . . . . . . . . . 32

3.2.1 System Model . . . . . . . . . . . . . . 32

3.2.2 SIR and Coverage Probability . . . . . . . . . 34

3.3 Key Prior Approaches . . . . . . . . . . . . . . 36

3.3.1 Lower Bound by Ignoring Holes: Approximating $\Psi$ by $\Phi_{2} 36$

3.3.2 Approximating the PHP by a PPP with the Same Density 37

3.3.3 Approximating the PHP by a PCP . . . . . . . . . 38

3.4 Proposed Approaches to Laplace Transform of Interference in the PHP . . . . . . . . . . . . . . . . . . . . . . . 42

3.4.1 Lower Bound on the Laplace Transform of Interference in the PHP . . . . . . . . . . . . . . 45

3.4.2 Upper Bound for the Laplace Transform of Interference in the PHP . . . . . . . . . . . . . 47

3.5 Incorporating the Impact of Overlaps in the Proposed Approaches 49

3.6 Numerical Results and Discussion . . . . . . . . . . . . 55

4 Serving Distance and Coverage in a PHP-Based Heterogeneous Cellular Network $\quad 68$

4.1 Introduction . . . . . . . . . . . . . . . . 68

4.2 System model . . . . . . . . . . . . . . . 70

4.3 Coverage Probability . . . . . . . . . . . . . 72

4.3.1 Distributions of serving distances $Z_{1}$ and $Z_{2} \ldots \ldots . \quad 72$

4.3.2 Coverage probability when the typical user is served by its closest macro cell . . . . . . . . . . . . 75

4.3.3 Coverage probability when the typical user is served by its closest small cell . . . . . . . . . . . . . . 77

4.4 Numerical Results and Discussion . . . . . . . . . . . . 79

5 Modeling and Analysis of Asymmetric Exclusion Zones 82

5.1 Introduction . . . . . . . . . . . . . . 82

5.2 Network Model . . . . . . . . . . . . . . . 85

5.2 .1 System Model . . . . . . . . . . . . 85

5.2 .2 SIR and Coverage Probability . . . . . . . . 86

5.2.3 Asymmetric Exclusion Zone in Infinite Network . . . . 88

5.2.4 Asymmetric Exclusion Zone in Finite Network . . . . . 89 
5.3 Numerical Results and Discussion . . . . . . . . . . . . . 93

6 Conclusion and Future Work 98

6.1 Summary . . . . . . . . . . . . . . . . . . 98

6.2 Future Direction . . . . . . . . . . . . . . . . . 101

6.2.1 More general performance metrics and fading distributions .................... 101

6.2.2 Arbitrary shaped exclusion zones . . . . . . . . . 103

6.2.3 Modeling CSMA-based network . . . . . . . . . . . . 104

6.2.4 Non-uniform user distribution and other applications . 105

6.3 List of Publications . . . . . . . . . . . . . . . . 106

$\begin{array}{ll}\text { References } & 107\end{array}$

$\begin{array}{lr}\text { Appendix A } & 115\end{array}$

A.1 Proof of Lemma 1 . . . . . . . . . . . . . . . . . 115

A.2 Proof of Lemma 5 . . . . . . . . . . . . . . 116

A.3 Proof of Theorem $2 \ldots \ldots 117$

A.4 Proof of Theorem $3 \ldots \ldots 117$

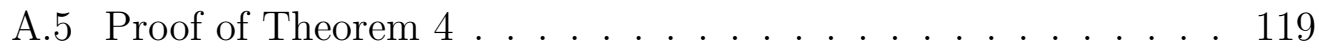

A.6 Proof of Theorem 5 . . . . . . . . . . . . . . . . . . 122

A.7 Proof of Proposition 1 . . . . . . . . . . . . . . . . . . . . 124

$\begin{array}{ll}\text { Appendix B } & 127\end{array}$

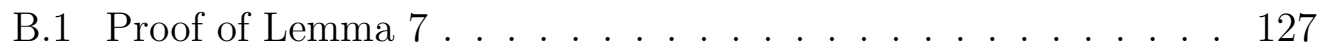

B.2 Proof of Theorem $6 \ldots \ldots \ldots$

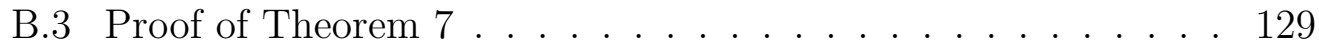

B.4 Proof of Theorem 8 . . . . . . . . . . . . 130

B.5 Proof of Theorem 9 . . . . . . . . . . . . . . 132

Appendix C 133

C.1 Proof of Theorem $10 \ldots \ldots$. . . . . . . . . . . . 133 


\section{List of Figures}

2.1 Two Independent Voronoi Tessellations (The filled squares with the solid Voronoi represent the macro tier) . . . . . . . 26

3.1 Illustration of the interference field with a single hole. . . . . . 42

3.2 Illustration showing that the closest point of $\Phi_{1}$ is at least a distance $D$ away from the typical node of the PHP $\Psi \ldots \ldots$. . 46

3.3 Illustration of the setup used in Theorem 4 where only two holes closest to the typical node are considered. . . . . . . . .

3.4 Illustration of the setup used in Theorem 5 where $k$ holes closest to the typical node are considered. . . . . . . . . . 51

3.5 The PHP network model (a) First configuration: LD-SH, (b) Second configuration: HD-SH, (c) Third configuration: LD-LH, (d) Fourth configuration: HD-LH. . . . . . . . . . . . 56

3.6 Ratio of the proposed upper and lower bounds derived in Theorems 3 and 2 , respectively. . . . . . . . . . . . . .

3.7 Comparison of g-functions of the Poisson hole process and the Thomas cluster process in LD-SH case $\left(\lambda_{1}=0.05\right.$ and $\left.D=0.6\right) .58$

3.8 Comparison of $\mathrm{g}$-functions of the Poisson hole process and the Thomas cluster process in HD-SH case $\left(\lambda_{1}=0.2\right.$ and $\left.D=0.6\right) . \quad 58$

3.9 Comparison of g-functions of the Poisson hole process and the Thomas cluster process in LD-LH case $\left(\lambda_{1}=0.05\right.$ and $\left.D=1.5\right) .59$

3.10 Comparison of g-functions of the Poisson hole process and the Thomas cluster process in HD-LH case $\left(\lambda_{1}=0.2\right.$ and $\left.D=1.5\right) . \quad 59$

3.11 Analytical and simulation results for the coverage probability as a function of the density $\lambda_{1}(D=1) \ldots \ldots \ldots$

3.12 Analytical and simulation results for the coverage probability as a function of the hole radius $D\left(\lambda_{1}=0.1\right) \ldots \ldots$. . . .

3.13 Analytical and simulation results for the coverage probability in LD-SH case $\left(\lambda_{1}=0.05\right.$ and $\left.D=0.6\right) \ldots \ldots . . .$. 
3.14 Analytical and simulation results for the coverage probability in HD-SH case $\left(\lambda_{1}=0.2\right.$ and $\left.D=0.6\right) \ldots \ldots$

3.15 Analytical and simulation results for the coverage probability in LD-LH case $\left(\lambda_{1}=0.05\right.$ and $\left.D=1.5\right) \ldots \ldots$.

3.16 Analytical and simulation results for the coverage probability in HD-LH case $\left(\lambda_{1}=0.2\right.$ and $\left.D=1.5\right) \ldots \ldots .$.

3.17 Analytical and simulation results for the coverage probabilities of the PHP nodes as a function of $\lambda_{2} / \lambda_{1}$ under configuration LD-SH $\left(\lambda_{1}=0.05\right.$ and $\left.D=0.6\right) \ldots \ldots \ldots \ldots$

3.18 Analytical and simulation results for the coverage probabilities of the PHP nodes as a function of $\lambda_{2} / \lambda_{1}$ under configuration HD-SH $\left(\lambda_{1}=0.2\right.$ and $\left.D=0.6\right) \ldots \ldots \ldots$

3.19 Analytical and simulation results for the coverage probabilities of the PHP nodes as a function of $\lambda_{2} / \lambda_{1}$ under configuration LD-LH $\left(\lambda_{1}=0.05\right.$ and $\left.D=1.5\right) . \ldots \ldots \ldots$

3.20 Analytical and simulation results for the coverage probabilities of the PHP nodes as a function of $\lambda_{2} / \lambda_{1}$ under configuration HD-LH $\left(\lambda_{1}=0.2\right.$ and $\left.D=1.5\right) \ldots \ldots \ldots$. . . . . .

4.1 Illustration of the PHP-based two-tier HCN system model. . . 70

4.2 Distribution of the distance from the typical user to its closest small cell BS in a PHP. . . . . . . . . . . . . . . 79

4.3 Comparison of macro and small cell coverage probability results. 79

4.4 Comparison of PPP macro and small cell and PHP-based HCN coverage probability results. . . . . . . . . . . . . 80

5.1 Modeling asymmetric exclusion zone (a) Infinite network; (b) Finite network. . . . . . . . . . . . . . . . . . 89

5.2 Comparison the outage probability of the PU between the secondorder Taylor series expansion and simulation results; Setup: $R=(20,25,500) m \ldots \ldots \ldots \ldots$

5.3 Impact of $\alpha$ on the outage probability of the user; Setup 1: $R=(25,250,500) m$; Setup 2: $R=(200,250,500) m \ldots 94$

5.4 Impact of $\alpha$ on the outage of the PU; Setup: $R=(10,25,500) m 95$

A.1 Illustration of the overlap effect for a typical hole in the PHP. The area of the region shaded in gray color is $\pi D^{2}-\frac{A_{\mathrm{ol}}}{2}$ and in red stripes is $A_{\mathrm{ol}}$. . . . . . . . . . . . . . . . . . . . . . 124 


\section{List of Tables}

3.1 Notation and Network Parameters . . . . . . . . . . . . . . . 34

5.1 Network Parameters of Asymmetric Exclusion Zone . . . . . . 93 


\section{List of Acronyms}

\section{Abbreviations Full Expressions}

$\begin{array}{ll}\text { ASE } & \text { Area Spectral Efficiency } \\ \text { BS } & \text { Base Station } \\ \text { CDF } & \text { Cumulative Density Function } \\ \text { CSMA } & \text { Carrier-Sensed Multiple-Access } \\ \text { D2D } & \text { Device-to-Device } \\ \text { FDD } & \text { Frequency Division Duplex } \\ \text { HDD } & \text { Hybrid Division Duplex } \\ \text { HCN } & \text { Heterogeneous Cellular Network } \\ \text { HetNet } & \text { Heterogeneous Network } \\ \text { PCP } & \text { Poisson Cluster Process } \\ \text { PDF } & \text { Probability Density Function } \\ \text { PHP } & \text { Poisson Hole Process } \\ \text { PINP } & \text { Poisson Point Process } \\ \text { TDD } & \text { Time-tnterference-plus-Noise Ratio } \\ & \end{array}$




\section{Chapter 1}

\section{Introduction}

In the last decade, the demand for wireless communication services has increased tremendously. The communication services comprise various types of applications like HD movie player, video conferencing and online games. This demand is especially strong for mobile communications to provide most of the new services continuously when users change their locations. Hence, the increasing trend leads to the deployment of wireless networks to satisfy throughput requirements of users. Meeting the demand for high data rates with traditional cellular network architectures is a crucial challenging issue. Heterogeneous networks (HetNets) are hierarchical architectures that are evolving in wireless networks [1-4]. They can be constructed by a combination of overlaid networks which have various technology requirements such as spectrum resource allocation, coverage area and power consumption.

Higher network throughput can be achieved by increasing spectrum us- 
age, spectrum efficiency and spectrum reuse. There is a restriction in the amount of accessible spectrum due to its limited resources. The improvement of networks by applying techniques like channel coding, multiple antennas and interference management is required to increase the spectral efficiency. The spatial reuse which is the basis of small-cell operation combined with two other methods achieves significantly higher network capacity $[5,6]$. The HetNet deployment targets the improvement of overall capacity as well as efficient coverage extension, and green radio solution. Hence, the HetNet planning can lead to more spatial reuse and alternatively would enhance usage of spectrum resources [7].

Small cell deployments have emerged as effective solutions in recent heterogeneous networks to increase capacity, enhance coverage and offload overlay network traffic [2]. These small and low power base stations which form tiered structures are either deployed by users arbitrarily (e.g. femtocells), or installed by operators (e.g. picocells). The two-tiered networks are deployed as the subset of hierarchical network architecture. This network consists of macrocell base stations as higher layer of networks that are underlaid with low-power small base stations such as femto access points. Two-tiered networks can decrease power consumption and also they have the potential of increasing spectrum efficiency and quality of service due to shorter distances between nodes [2]. Small cells reuse spectrum aggressively to enhance spectral efficiency in HetNets. However, one of the most critical issues in spectrum 
sharing schemes is the potential interference between adjacent small cells as well as the interference between small cells and macrocells with common subcarriers. These undesired effects are called co-tier interference and cross-tier interference respectively which mitigate the overall system capacity. Hence, modeling and overcoming interference is one of the critical challenges in tiered networks. Stochastic geometry has been proposed as a powerful tool for the modeling and analysis of interference distributions while it captures spatial randomness of small cells in tiered HetNets [8,9].

Using radio spectrum efficiently as a scarce and valuable resource can be achievable by employing different spectrum access policies. They aim to increase spectrum efficiency which consequently leads to capacity improvement of wireless networks. It is notable that among different schemes, networks can gain higher capacity through spectrum reuse and adding more nodes into the system. Since small cells are often distributed randomly, the implementation of centralized spectrum access has some challenges due to the lack of information. Therefore, distributed spectrum access methods that can be organized in each cell are more appropriate in these networks [10]. These spectrum access approaches can be categorized as either cooperative or noncooperative ones. In non-cooperative approaches, base stations (BSs) have selfish behaviour such that they optimize their own throughput at the expense of imposing more interference to adjacent cells. In cooperative approaches, BSs collect some information about their neighborhood for maximizing their 
own throughput while keeping the level of interference to adjacent cells below a certain threshold. Cooperation between different tiers of HetNet can lead to more spectrum sharing advantages [11].

The infrastructure of backhaul that connects different types of BSs and nodes has impact on the the performance gain of communication networks [12]. This structure can be based on two models: wired backhaul and wireless backhaul. There are various types of technologies (fiber-optic, microwave, etc.) in each of these groups with their own advantages and disadvantages. In dense networks, exchanging information between BSs either in the same tier or between different tiers increases signaling overhead. IP based backhaul is more common in small cells but has some drawbacks like capacity limitation and delay transmission. In 3GPP Long Term Evolution (LTE), X2 interfaces that connect macro BSs play the role of backhaul in the networks. They can transmit scheduling information and interference information too [13].

Self organizing networks allow autonomous configuration and optimization of the networks whereby they can adapt themselves dynamically to varying situations. The self organization techniques enable networks for managing cotier and cross-tier interference without requiring X2 interface. For instance, busy tone signaling is applied for mitigating interference in time division duplexing networks in [14]. Further, cognitive capability which enables small cells to collect some information about their environment can be used to avoid harmful interference to adjacent cells [15]. In general, a cognitive user can take 
advantage of three dimensions i.e. time, frequency and space as long as there is no harmful interference from secondary network to primary network. This condition happens when either there is no primary user transmission or the transmission occurs at a different frequency band or in far distance. In order to control interference, cognitive enabled small cells and users can sense operating environment of themselves (e.g. by sensing control channels of macro tier). Cognitive capability or exchanging some beacon signals such as busy tone enables small cells to configure self organized network.

Network simulation can study various actual scenarios at any desired depth of detail, but it has some drawbacks too. Separate simulations are required for different scenarios and for each choice of design parameters. By increasing the tiers of the network, the number of network parameters that should be combined rises exponentially which leads to more complexity. Extending the results obtained from known scenarios to new ones is not possible in all cases of interest due to variety of network deployments. Stochastic geometry approaches are able to develop tractable frameworks for the modeling and analysis of wireless networks. 


\subsection{Interference Management Techniques in}

\section{Wireless Networks}

Motivated by the importance of interference management in wireless networks, a large body of research has focused on this field. Main types of studies are classified into two groups [16]: statistical approaches and instantaneous approaches. In instantaneous approach, optimization of objective functions which are modeled by using available instantaneous information of the system is desirable. These objective functions can cover a large variety of resource allocation (e.g., channel allocation, power allocation) problems [17-22]. In this work, our focus is on the statistical approach which is based on some statistical information like distributions of transmitter and receiver nodes and channel gains. Further, it has higher stability compared to instantaneous approach with fast varying parameters.

There are some studies on ad hoc networks in which stochastic geometry approaches are applied [23-28]. In [29], interference cancellation scheme is applied for an ad hoc network by considering either closest or strongest interfering node. Then, spectrum sharing transmission capacity in two different coexisting systems is obtained. In [30], a cognitive network is considered which in secondary cognitive transmitters are not allowed to be active within the circular regions around primary receivers. Active cognitive transmitters form a doubly Poisson point process that has similar properties to Poisson 
cluster process. Then, some bounds are derived for the outage probability of this system in the bipolar ad hoc network. In [31], the hard core point process which can capture stronger correlation between nodes is used for modeling a non-cellular network. A lower bound is derived for the outage probability of a random carrier-sensing multiple-access (CSMA) network by considering dominant interfering nodes. In [32], the effect of mobility on the interference and outage statistics in a random network is evaluated by considering different mobility models. Further, the temporal correlation of interference and outage due to mobility is obtained in this work.

There are some related works with common themes of stochastic geometry and interference cancellation and mitigation in HetNet. In [33], the network model consists of only one macro base station, one macro user and cognitive small cells. A coordination mechanism is applied to manage cross-tier interference. Then, the outage probability and average channel capacity of the network are obtained by considering shifted log-normal approximations. In [34], cognitive femtocell BSs use CSMA protocol to prevent reusing any used channel in macro and femto spectrum sensing region. The performance gain of outage probability corresponding to this system is wasted due to the existence of some unavailable channels in the spectrum sensing process. In [35], some ALOHA schemes based on sensing information at small cells are applied to mitigate co-tier and cross-tier interference. In [36], stochastic geometry models enable the analysis of a multi-tier network with flexible cell association. 
Here, users are biased to BSs which have highest averaged received power. The average number of users associated to each tier is obtained through pertier association probability. Then, the outage probability, the average ergodic rate, and the average user throughput are obtained. The later metric measures the quality of service (QoS) that the network can provide after biasing.

In [37], cognitive enabled small cells adapt their transmission by decoding macro BS information to achieve higher spectrum efficiency. The information includes location, rate and transmit power of the macro BS. Two strategies are proposed to mitigate interference from small cells to macrocell. The first strategy is based on forming a region around macro user with constant radius wherein small cells shut-down their transmission. The second one is through controlling the transmission power of small cells. To further enhance throughput, downlink interference alignment is also applied. In this work, random distribution of small cells is not considered and network analysis is performed over a grid-based model.

In [38], two types of small cell users are considered which operate under open access or closed access policy. This type of access policy is recognized as the hybrid-access policy. Small cells are distributed according to the PPP and also Neyman Scott cluster process. The SINR distribution of a two-tier network is derived in a multi-carrier multi-channel system. There are some non-occupied sub-channels in this system that differentiates it from common analysis due to not considering full network load. In [39], interfering nodes 
which are distributed outside a fixed size cell according to PPP is approximated by gamma distribution. This work is one of the pioneer efforts on multi-antenna HetNet. In $[40,41]$ cognitive radio technique is applied for the spectrum sharing in a two-tier network. Cognitive enabled small cells which work in the TDD mode on both uplink and downlink FDD bands of macrocell can control interference by sensing control channels corresponding to macrocell. They access the FDD bands of macrocell opportunistically to operate in the uplink and downlink mode. The effect of cross-tier interference on macrocell is controlled by the self configuration approach. This work provides a general framework for the analysis of the interference distribution in simultaneous uplink and downlink transmission but without applying any interference management technique.

In [42], uplink transmission in a single tier network is considered which in one BS is located in each Voronoi cell of the user. Channel inversion power control is considered in this analysis. This work is extended to a multiuser uplink cellular system in [43] based on conditional thinning. In [44], a new analysis has been conducted on uplink scenario for multi-tier networks. Here the Voronoi cell is established around BSs and truncated power control is employed by users to keep average received power equal to the threshold which further control the level of interference.

Coordinated Multi-Point transmission (CoMP) for LTE is one of the techniques which is used in new HetNet deployment and can further contribute 
to interference management. In [45], two cooperative BSs serve a typical user while the signal is split to a common part sent by cooperating pairs and a private part that chooses no cooperation. Here, cooperation is achieved by certain exchange of information between two neighboring nodes. This approach is based on conferencing over backhaul link. The decision to cooperate or not depends on the ratio of the distances between the first and second neighboring nodes to user.

\subsection{Related Works and Motivation}

PHP-based models have already been adopted for the analysis of networks in which holes (also called exclusion zones) are created around nodes (e.g., BSs, devices, sensors, and etc.) that need to be protected from excessive interference. In some applications such as cognitive radio, heterogeneous cellular and device to device networks, the PHP turns out to be a reasonable model for network modeling and performance analysis. For instance, the main objective of cognitive radio networks is to improve spectrum utilization by allowing unlicensed secondary users to use licensed spectrum as long as they do not cause excessive interference to the licensed primary users. One way to ensure this is by creating exclusion zones (holes) around primary users, where secondary transmitters are not allowed to transmit within exclusion zones. Hence, the cognitive radio network can be modeled by a PHP, as already 
done in [30]. Similarly, heterogeneous cellular networks usually exhibit spatial separation amongst base stations (BSs) of different types. For instance, operators will usually not deploy a picocell in close proximity to a macrocell, and hence there is spatial separation between the locations of pico and macro BSs. This spatial separation can also be modeled by a PHP, where the macro BSs are assumed to be located at the center of holes, as done in [46-48]. On similar lines, to protect cellular network from excessive D2D interference, no D2D transmissions are allowed in vicinity of cellular receiver, and hence PHP is a good candidate for the analysis of the resulting setup, as done in [16,49]. Further, PHP is also used to study cognitive D2D communication in cellular network when transmitters are powered by harvesting energy from the ambient interference in [50]. Finally, in [51], a Poisson Cluster Process (PCP) and a PHP are merged to develop a new spatial model for integrated D2D and cellular networks. In particular, a modified Thomas cluster process is used to model device locations where instead of modeling the cluster centers as a homogeneous PPP, they are modeled as a PHP.

In summary, while there are numerous applications of PHP in current wireless networks, we are somewhat short handed when it comes to the analytical tools to handle the analysis of such scenarios formally. This work provides a comprehensive framework to facilitate this analysis. The contribution therefore is not necessarily targeted to a particular application. It is to develop mathematical tools for the analysis of wireless networks modeled as a PHP. 
Wireless networks can also establish exclusion zones to protect the communication of the user at any arbitrary location inside the cell. This model is applicable for different network setups like IEEE 802.22 Digital TV [52][54], IEEE 802.11 network which considers carrier sense multiple access with collision avoidance [55], networks which in CSMA protocol leads to Matérn hard core process [56]- [57], device-to device communication in cellular networks [49]- [50]. This realistic model is unlike most existing works in which the symmetric exclusion zones protect the typical user located at the center of the cell [58]- [60]. In this work, we provide a new tractable analytical framework for the modeling and analysis of this system.

\subsection{Contributions and Outcomes}

The main contributions of this dissertation are summarized next.

New approach to the analysis of the PHP. Unlike existing approaches that approximate the PHP with either a PPP or a PCP (such as Thomas or Matérn cluster processes), we develop a new approach that is amenable to shot-noise analysis and leads to tight provable bounds on the Laplace transform of interference experienced by a typical node of the PHP. A lower bound is first derived by overestimating interference by ignoring all the holes except the closest one. We provide an equivalent interpretation of this result in which the closest hole is dissolved in such a way that it results in a tractable non- 
homogeneous PPP. The resulting bound is shown to be remarkably tight. Extending this approach to multiple holes, we derive an upper bound on the Laplace transform of interference by carving out each hole separately without accounting for the overlaps between them. This leads to the removal of some points from the baseline PPP multiple times, thus underestimating the interference power experienced by the typical node. This bound is also shown to be remarkably tight across a variety of scenarios, including the ones in which the holes exhibit significant overlaps $[61,62]$.

Approaches to incorporate the effect of overlaps in the holes. In the first set of bounds discussed above, we carefully circumvented the need for incorporating the effect of overlaps between holes. While these simple and easy-to-use bounds are tight, we also provide ideas for incorporating the overlaps between holes, which tighten these bounds even further. In the first part, we generalize the lower bound discussed above by considering two closest holes in the interference field while incorporating the exact effect of overlap between them. In the second part, instead of trying to incorporate the exact effect of overlaps, we propose a new procedure for bounding the overlap area, which allows us to derive a provable lower bound on the Laplace transform while considering multiple holes in the interference field. In the third and final part, we propose a new approach that allows to incorporate the mean effect of overlaps in the holes [61].

New insights. Our results concretely demonstrate that for accurate analysis 
of interference in the PHP, it is very important to preserve the local neighborhood around the typical node. For instance, we show that considering even a single hole in the interference field results in a tighter characterization of interference power at the typical node of the PHP compared to seemingly more refined prior approach of first-order statistic approximation in which the PHP is approximated by a PPP with the matching density. This is because by considering a single hole, the local neighborhood around the typical node is accurately captured, whereas it is distorted in the other approach due to independent thinning involved in the density matching of a PPP. Numerical results also reveal that our first set of bounds derived without incorporating the effect of overlaps, while being seemingly simple, are so tight that the additional complication in the expressions resulting from more sophisticated analysis of overlaps is not commensurate with the gains [61].

The second contribution is to consider a two-tier closed-access PHP-based HCN model, where the locations of the macro BSs are modeled by a PPP and those of small cell BSs by a PHP. This naturally captures the inter-tier spatial separation. To enable the downlink analysis of this model, we first derive a bound on the distance of a typical user to the closest point of a PHP using general approach of preserving the local neighborhood proposed by the approach in chapter 3. Numerical comparisons show that the bound is remarkably tight across wide range of system parameters. This gives us the serving distance distribution when a typical user connects to the small cell 
tier. We then derive several new bounds and approximations for the coverage probability for the closed access case where the typical user is authorized to connect to one of the two tiers (either macro or small cells) [63].

Our third contribution is to provide a new tractable analytical framework for the modeling and analysis of the systems in which asymmetric exclusion zones are established. The proposed approach is based on the transformation from asymmetric interference field with homogeneous PPP into the symmetric one with the non-homogeneous PPP. We also apply this transformation for a finite network. We derive accurate expressions for the outage probability of the system using stochastic geometry. The proposed approach is applicable in diverse deployment scenarios in wireless networks which encompass the exclusion zones and asymmetric interference field. Simulations verify that the proposed model provides an accurate way to characterize the outage probability of the system.

\subsection{Organization of the Thesis}

The layout of this thesis is as follows. In chapter 2, we present mathematical preliminaries and fundamental tools for network modeling based on stochastic geometry approaches. First, we provide an overview on point process models. Second, we discuss on different approaches for the network performance analysis. Third, we present some basic concepts for stochastic ge- 
ometry modeling. In chapter 3 , we focus on the analysis of the Poisson hole process-based model which has been adapted as a reasonable model for the performance analysis of some applications in current wireless networks. First, we study previous bounds and approximations for the PHP-based models. Further, we obtain the results corresponding to the interference distribution of PHP which is approximated by Poisson cluster processes (PCP). We develop a new approach for the analysis of the PHP-based models and derive some new bounds and approximations. Then, we develop new approaches to incorporate the effect of overlaps in the holes. We compare our proposed bounds and the new approximation with the prior approaches. In chapter 4, we study a Heterogeneous cellular network ( $\mathrm{HCN})$ which is modeled by a PHP. First, we derive a new bound for the serving distance distribution in the presence of holes. Then, we propose a new approach for the analysis of the PHP-based HCN and derive new bounds and approximations for the coverage probability of a typical user in this system. In chapter 5 , we focus on the analysis of the asymmetric exclusion zone. We develop a novel approach for the modeling and analysis of this system and derive the coverage probability of the typical user which is arbitrary distribute inside the cell. We consider two cases which the first one is the asymmetric exclusion zone in infinite network and the second one in finite network. In chapter 6 , the conclusion and future direction are presented. 


\section{Chapter 2}

\section{Overview of Network Modeling}

A practical mathematical model can assess many important characteristics of wireless networks like channel access probability, coverage probability and average throughput. Stochastic models can display interactions between users and different transmitters of the network. In real deployments, nodes are usually distributed randomly especially in unplanned networks. One appropriate realization of a random point process for the locations of nodes is homogeneous Poisson point process (PPP) [55]. In this realization, nodes are distributed independently and uniformly which leads to the analytical tractable model since the derivation of closed form expressions compared to other types of point processes is much easier.

Until recently, the mathematical performance of the networks were evaluated based on simplified models such as Wyner model and deterministic grid-based models which fail to capture the characteristics of the current de- 
ployment trends in wireless networks. For interference characterization, these models lead to either inaccurate or intractable results due to unrealistic assumptions [64]. Moreover, grid based model can not capture the random deployment of nodes especially in $K$ tier networks. In these networks, stochastic geometry has the potential of capturing randomness of nodes in the network and leads to analytically tractable models. A rich mathematical tool is provided by stochastic geometry which can average over many network realizations to characterize network performance parameters such as SINR, outage probability and rate coverage.

Spatial models can develop simple frameworks for optimizing network parameters. They provide neat analytical results and insightful spatial averages of key performance metrics. However, they have several limitations and shortcomings. In realistic scenarios, the locations of the nodes are not independent. In addition, temporal correlations among the nodes arises due to mobility. Different classes of point processes can display various factors such as network topology and interactions between the nodes. The main problem about some of these models is that they are often not analytically tractable. Further, stochastic geometry provides spatial averages of the network performance metrics while may ignore the effect of the design parameters on the uncertainties caused by random deployments. 


\subsection{Point Process Models}

A point process is a kind of random process taking values in the $n$-dimensional Euclidean space $\mathbb{R}^{n}$ which can capture the network properties. In stochastic geometry, points play fundamental roles and spatial patterns are studied as a system of points. The various random point patterns result from various kinds of interaction between their points. The patterns can be clustered or more regular than a PPP. They also may have a minimum inter-point distance. In this section, some types of point process models are introduced. Before studying different types of point processes, we first give some basic definitions [65].

Simple Point Process. Let $N$ be a set of all sequences of points $\phi \subset \mathbb{R}^{n}$ which is locally finite, i.e., the bounded set $B \subset \mathbb{R}^{n}$ contains finite number of points. Further, a point process is called simple if its points are not colocated, i.e., $x_{i} \neq x_{j} \quad \forall i \neq j$. A simple point process is a random variable taking values in the space $N$.

Campbell's theorem. Let $f: \mathbb{R}^{n} \rightarrow[0, \infty]$ be a measurable function over a point process $\Phi$ and $\Lambda(B)$ is the intensity of the point process. Then

$$
\mathbb{E}\left[\sum_{x \in \Phi} f(x)\right]=\int_{\mathbb{R}^{n}} f(x) \Lambda(\mathrm{d} x) .
$$

For the stationary point process with density $\lambda$, the right side is equal to $\lambda \int_{\mathbb{R}^{n}} f(x) \mathrm{d} x$. 
Probability generating functional (PGFL). Let $f: \mathbb{R}^{n} \rightarrow[0, \infty]$ be a measurable function over a point process $\Phi$ and $\Lambda(B)$ is the intensity of the point process. Then,

$$
\mathbb{E}\left[\prod_{x \in \Phi} f(x)\right]=\exp \left(-\int_{\mathbb{R}^{n}}(1-f(x)) \Lambda(\mathrm{d} x)\right)
$$

\subsubsection{Poisson Point Processes}

In the case of the homogeneous Poisson point process, intensity measure $\Lambda$ is proportional to Lebesgue measure. Lebesgue measure is equal to length measure in $\mathbb{R}^{1}$, area measure in $\mathbb{R}^{2}$ and volume measure in $\mathbb{R}^{3}$. A point process is a PPP if the number of nodes in a bounded set $B$, is a Poisson random variable and there is no interaction between points, i.e., they are independent. Moreover, PPP provides a parent model for the definition of different types of point process models.

In a stationary Poisson point process $\Phi$, the number of points in the bounded set $B \subset \mathbb{R}^{n}$ with intensity measure $\Lambda(B)=\int_{B} \lambda(x) \mathrm{d} x \lambda(x)$ is as follows [65]

$$
\mathbb{P}(\Phi(B)=n)=\exp (-\Lambda(B)) \frac{\Lambda(B)^{n}}{n !}, \text { for } n=0,1,2, \ldots
$$

The number of points in $B_{1}, B_{2}, \ldots, B_{k}$ when they form $k$ disjoint bounded sets, are independent random variables. 


\subsubsection{Hard-core Processes}

Hard core point process (HCPP) is a point process which in the points have a certain minimum distance, i.e., they are forbidden to be closer than this predefined separating distance.

Matérn hard-core processes. Two models are introduced as the Matérn hard-core processes of type I and II [66]. Matérn hard-core process of type I is obtained by removing all the points located within certain distance $D$ from a uniform PPP with density $\lambda_{\mathrm{b}}$. In Matérn hard-core process of type II, an independent random variable $m(\mathbf{x})$, called a mark, uniformly distributed on $[0,1]$ is associated to each point of uniform $\mathrm{PPP}, \Phi_{\mathrm{b}}$, independently. This point process that has a smaller mark is obtained by removing all points located in the neighborhood with radius $D[67]$.

$$
\Phi \triangleq\left\{\mathbf{x} \in \Phi_{\mathrm{b}}: m(\mathbf{x})<m(\mathbf{y}) \quad \forall \mathbf{y} \in \mathbf{b}(\mathbf{x}, D) \cap \Phi_{\mathrm{b}} \backslash\{\mathbf{x}\}\right\}
$$

where $\Phi_{\mathrm{b}}, \mathcal{C}=\mathbf{b}(\mathbf{x}, D)$ and $m(\mathbf{x})$, respectively denote a uniform PPP with density $\lambda_{\mathrm{b}}$, a ball of radius $D$ centered at $\mathbf{x}$ and a mark which is an independent random variable uniformly distributed on $[0,1]$.

The density of the hard-core process of type $\mathrm{I}$ is $\lambda=\lambda_{\mathrm{b}} \exp \left(-\lambda_{\mathrm{b}} \pi D^{2}\right)$. Conditioned on the point $\mathbf{x}$ having a mark $t \in[0,1]$, the probability that $\mathbf{x}$ is selected equals $\exp \left(-t \lambda_{\mathrm{b}} \pi D^{2}\right)$ wherein $t \lambda_{\mathrm{b}}$ is the density of points with marks smaller than $t$. To determine the density of the hard-core process of type II, 
by averaging over $t$,

$$
p=\int_{0}^{1} \exp \left(-t \lambda_{\mathrm{b}} \pi D^{2}\right)=\frac{1-\exp \left(-\lambda_{\mathrm{b}} \pi D^{2}\right)}{\lambda_{\mathrm{b}} \pi D^{2}}
$$

So the final density of the process equals $\lambda=\lambda_{\mathrm{b}} p$.

\subsubsection{Cluster Processes}

The Poisson cluster process is constructed by taking a parent point process in which each point is replaced by a cluster of points. The daughter points of the cluster are independent and identically distributed around each parent point. The Poisson cluster process is the superposition of all clusters. We further discuss about this process in chapter 3 .

\subsubsection{Cox Processes}

A general Poisson point process which in the intensity measure itself is a random variable is doubly stochastic Poisson process or Cox process [66]. The Cox process behaves like an inhomogeneous Poisson process. Poisson hole process is defined as a subset of Cox process model. Since forming holes leads to node concentration in some areas, there is a similarity between Poisson hole process behaviour and Poisson cluster process. 


\subsection{Network Performance Analysis}

In this section, some basic definitions and mathematical preliminaries for stochastic geometry modeling, analysis and design of wireless networks are introduced. Network modeling affects interference statistics as well as the SINR statistics subsequently. The SINR experienced by the test receiver is defined as

$$
S I N R=\frac{P_{t} h r^{-\alpha}}{W+\sum_{y \in I} P_{t} h|y|^{-\alpha}}
$$

where $W$ is the noise power and $I$ is the set of active transmitters that transmit on the same channel. Interference is one of the key network parameters. Further, network characteristics like user association and spectrum access methods have significant impact on the interference statistics. Aggregate interference of the network is a stochastic parameter which depends on the distribution of interfering nodes and channel gains of the network. Interference can be characterized by either its PDF or its CDF. However, in some cases like general path-loss exponents or large scale networks, the PDF cannot be expressed in closed-form. Hence, interference can be characterized by using Laplace transform (LT) of PDF (equivalently its characteristic function or moment generating function (MGF)). Obtaining exact expressions for the network performance metrics depends on the type of point process models and only approximate expressions are achievable in some cases. Different 
techniques are utilized to quantify network performance by using LT for the interference [68].

One of the most popular techniques for the derivation of the PDF of aggregate interference is based on considering Rayleigh fading on the desired link between transmitter and receiver. This assumption often leads to accurate expressions for the CDF of SINR. Laplace transform of interference is a common technique for obtaining the CDF of interference which itself is useful in evaluating transmission capacity, success probability and achievable data rate. This technique works well under the assumption of Rayleigh fading channel gains but it is not an efficient technique for general fading models.

The second technique for evaluating interference statistics is achievable by bounding the interference. One approach is based on considering the $n$ closest interfering nodes. Another one is derived by considering the effect of dominant interfering nodes located in the region around the typical node with received power above a threshold. Both of these approaches leads to the lower bounds for the outage probability which in some conditions (higher values of pathloss exponent) are tight bounds. An upper bound for the outage probability can be evaluated by Chernoff, Markov and Chebyshev bounds. However, the lower bound derived by the first types of approaches often leads to the tighter results [69].

Approximating the interference distribution by one of the known PDFs like gamma, log-normal, shifted log-normal distributions is another popular tech- 
nique for quantifying aggregate interference [70]. This approach works better in the environments with lower path-loss exponent. In summary, applying one of the common proposed techniques for obtaining aggregate interference characteristics depends on the type of network, point process models, fading channel and even the value of path-loss exponent.

\subsection{Stochastic Geometry Modeling for Tiered}

\section{Networks}

Current cellular networks are becoming unplanned, decentralized and heterogeneous. In these networks, cells are smaller with more random distribution as well as more chaotic behavior. Modeling the cellular network with HCPP is more realistic because of satisfying the minimum distance constraint between nodes. However, in terms of analytical tractability, this model compared to the PPP is more complicated. In heterogeneous networks, the locations of the BSs in different tiers of the network can be modeled by independent homogeneous PPPs which form the Voronoi tessellation. This concept is described in below definitions.

Definition 1 Voronoi cell. The Voronoi cell $V(\mathbf{x})$ of a point $\mathbf{x}$ from a general point process $\Phi \subset \mathbb{R}^{n}$ is formed by all the points of $\mathbb{R}^{n}$ which are closer to $\mathbf{x}$ 


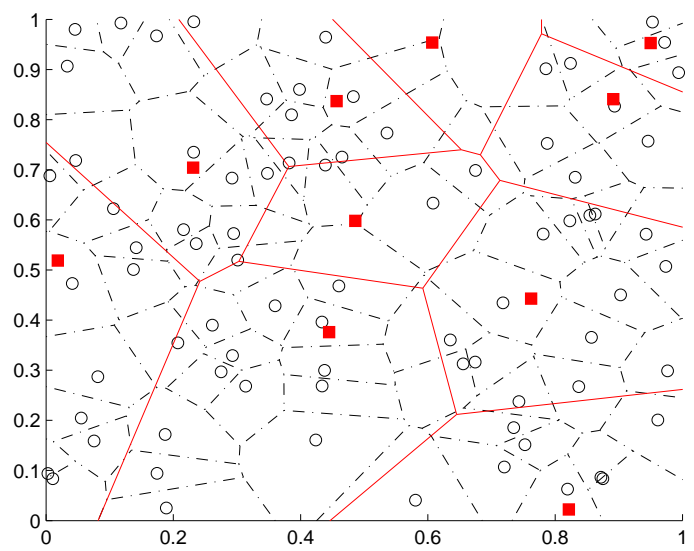

Figure 2.1: Two Independent Voronoi Tessellations (The filled squares with the solid Voronoi represent the macro tier)

than to any other points of the point process [67].

$$
V(\mathbf{x}) \triangleq\left\{\mathbf{y} \in \mathbb{R}^{n}:\|\mathbf{x}-\mathbf{y}\| \leqslant\|\mathbf{z}-\mathbf{y}\| \quad \forall \mathbf{z} \in \Phi \backslash\{\mathbf{x}\}\right\}
$$

Definition 2 Voronoi tessellation. The superposition of all these Voronoi cells, $V(x) s$, forms Voronoi tessellation. The locations which are equidistant from two points of the PPP form boundaries of the tessellation while their intersections are equidistant from three points of the PPP.

In figure 2.1, two independent Voronoi tessellations are depicted. Here, filled squares with the solid Voronoi represent a macro cell while hollow circles with dashed Voronoi represent a small cell. A two tier-network can be modeled by the superposition of the two tessellations such that the coverage of each tier forms a Voronoi tessellation.

The mathematical tools provided in this chapter are applied for modeling 
and analysis of interference management techniques in the next sections. 


\section{Chapter 3}

\section{Poisson Hole Process: Theory}

\section{and Applications to Wireless}

\section{Networks}

\subsection{Introduction}

The received signal-to-interference-plus-noise ratio (SINR) is known to be a strong indicator of the performance and reliability of a wireless link. Several key performance metrics of interest, such as outage probability, ergodic capacity, and outage capacity, are strongly dictated by the received SINR. By definition, the SINR distribution depends upon the joint distribution of the received powers from the serving node and the interfering nodes, which ulti- 
Chapter 3: Poisson Hole Process: Theory and Applications to WIRELESS NETWORKS

mately depend on the network topology. Therefore, accurate modeling of the network topology becomes a key step towards meaningful performance analysis of wireless networks. Owing to its tractability and realism in modeling irregular node locations, stochastic geometry has emerged as an important tool for the realistic analysis of wireless networks $[24,67,71,72]$. Initially popular for the modeling of wireless ad hoc and sensor networks, e.g. see [69,70], it has recently been adopted for the analysis of cellular and heterogeneous cellular networks as well $[9,73,74]$. Irrespective of the nature of the wireless network, the interference field is almost always modeled by a homogeneous PPP to maintain tractability. While this leads to remarkably simple results for key performance metrics, such as coverage and rate, it is not quite suitable for modeling the effect of interference management techniques, which often introduce some form of spatial interaction among transmitters. In this chapter, we focus on spatial separation, where holes (also called exclusion zones) are created around nodes/networks that need to be protected from excessive interference [53]. In particular, we assume that the baseline interference field is a PPP from which holes of a given radius are carved out. When the locations of the holes also form an independent PPP, the resulting point process is usually termed as the PHP, which is the main focus of this chapter. 


\subsubsection{Related Work and Applications}

We first discuss a few of possibly numerous instances in wireless networks where the PHP as a stochastic process is a more appropriate model for node locations. In particular, we discuss how the PHP has been used to model cognitive radio networks, heterogeneous cellular networks, and device-to-device (D2D) networks. In cognitive radio networks, unlicensed secondary users are allowed to use licensed spectrum as long as they do not affect the performance of the licensed primary users. This can be achieved by creating exclusion zones (holes) around primary users, where secondary transmissions are not allowed. This spatial separation was elegantly modeled by using the PHP in [30]. In particular, the locations of both primary and secondary users were first modeled by independent PPPs. Assuming secondary transmissions were not allowed within a given distance from the primary users, the locations of active secondary users were then modeled by the PHP.

The PHP has also been used recently to model inter-tier dependence in the base station locations in a heterogeneous cellular network in [46-48]. Modeling the macrocell locations by a PPP, it was assumed that the small cells are deployed farther than a minimum distance from the macrocells, i.e., outside exclusion zones of a given radius. In such a case, small cells form the PHP. This model introduces repulsion between the locations of macro and small cells, which is desirable due to several reasons, such as interference mitigation at macrocells due to small cell transmissions, and the higher advantage of 
Chapter 3: Poisson Hole Process: Theory and Applications to WIRELESS NETWORKS

deploying small cells towards the cell edges of macrocells, especially in the coverage-centric deployments.

Similarly, for underlay D2D communication in cellular networks, inhibition zones may be created around cellular links where no D2D transmissions are allowed, thus saving cellular links from excessive D2D interference. The active D2D transmitters outside the holes form the PHP $[16,49]$. In this regard, cognitive D2D communication in cellular network when transmitters are powered by harvesting energy from the ambient interference is studied in [50]. In [51], a Poisson Cluster Process (PCP) and a PHP are merged to develop a new spatial model for integrated D2D and cellular networks. In particular, a modified Thomas cluster process is used to model device locations where instead of modeling the cluster centers as a homogeneous PPP, they are modeled as the PHP to account for the inhibition zones around cellular links.

Despite the importance of the PHP in modeling wireless networks, the exact characterization of interference experienced by a typical receiver in the PHP is a challenging problem. There are two main directions taken in the literature for the analysis of wireless networks modeled by PHPs. The first approach, termed first-order statistic approximation, approximates the PHP by a homogeneous PPP with the same density [67]. The second approach ignores the holes altogether to approximate the PHP by its baseline PPP. This overestimates the interference and the accuracy of the approximation is a function of system parameters $[16,30,49,50]$. Besides, the PHP is sometimes approxi- 
mated with a PCP by matching the first and second order statistics $[30,46,47]$.

The resulting expressions for performance metrics are usually more complicated in this case compared to the above two. While all these approaches are reasonable, they are typically not accurate beyond a specific range of system parameters. In this chapter, we take a fresh look at this problem and derive tight upper and lower bounds for the Laplace transform of interference experienced by the typical node in the PHP.

\subsection{Network Model}

\subsubsection{System Model}

We consider a wireless network that is modeled by the PHP in $\mathbb{R}^{2}$. The PHP can be formally defined in terms of two independent homogeneous PPPs $\Phi_{1}$ and $\Phi_{2}$, where $\Phi_{2}$ represents the baseline PPP from which the holes are carved out and $\Phi_{1}$ represents the locations of the holes. Let the densities of $\Phi_{1}$ and $\Phi_{2}$ be $\lambda_{1}$ and $\lambda_{2}$, respectively, with $\lambda_{2}>\lambda_{1}$. Denoting the radius of each hole by $D$, the region covered by the holes can be expressed as

$$
\Xi_{D} \triangleq \bigcup_{\mathbf{y} \in \Phi_{1}} \mathbf{b}(\mathbf{y}, D), \quad \mathbf{b}(\mathbf{y}, D) \equiv\left\{\mathbf{z} \in \mathbb{R}^{2}:\|\mathbf{z}-\mathbf{y}\|<D\right\}
$$


Chapter 3: Poisson Hole Process: Theory and Applications to

The points of $\Phi_{2}$ lying outside $\Xi_{D}$, form the PHP, which can be formally expressed as

$$
\Psi=\left\{\mathbf{x} \in \Phi_{2}: \mathbf{x} \notin \Xi_{D}\right\}=\Phi_{2} \backslash \Xi_{D}
$$

It should be noted that the PHP $\Psi$ has also been known as a Hole-1 process in the literature [75].

We characterize the interference experienced by a randomly chosen node $\mathbf{x} \in \Psi$ due to the transmission of the other nodes of $\Psi$. For a given $\Xi_{D}$ (i.e., given $\Phi_{1}$ ), by Slivnyak's theorem we can treat this randomly chosen observation point (node) $\mathbf{x}$ as an additional point of the PPP of density $\lambda_{2}$ defined on $\mathbb{R}^{2} \backslash \Xi_{D}[67]$. Now by stationarity of the PHP, we can translate $\Psi$ such that this random observation point coincides with the origin. We call this point the typical node of PHP. Note that by construction, this typical node is located outside the holes, which means there are no points of $\Phi_{1}$ within a disk of radius $D$ around the origin. In other words, this means that the above choice of the observation point conditions on the Boolean model $\Xi_{D}$ that does not cover the origin. We consider simple ad hoc setup which in transmitter is located at the fixed distance $r_{0}$ from its receiver. It should be noted that we could have considered more sophisticated models for the serving link of interest but we chose to consider this simple setup because our emphasis is on characterizing interference in the PHP. For the wireless channel between points 
Table 3.1: Notation and Network Parameters

\begin{tabular}{l|l}
\hline \hline Symbol & Description \\
\hline$\Phi_{1} ; \lambda_{1}$ & Independent PPP modeling the locations of hole centers; density of $\Phi_{1}$ \\
$\Phi_{2} ; \lambda_{2}$ & Independent PPP from which the holes are carved out; density of $\Phi_{2}$ \\
$\Psi$ & PHP formed by carving out holes with centers $\Phi_{1}$ from $\Phi_{2}$ \\
$D$ & Radius of each hole carved out from $\Phi_{2}$ \\
$\mathrm{P}_{\mathrm{c}}, \gamma$ & Coverage probability (in terms of SIR); SIR threshold \\
$\mathcal{L}_{I}(s)$ & Laplace transform of $I$, defined as $\mathbb{E}\left[e^{s I}\right]$ \\
$\mathcal{C}=\mathbf{b}(\mathbf{y}, D)$ & Ball of radius $D$ centered at $\mathbf{y}$ \\
$h_{\mathbf{x}}$ & Fading gain $h_{\mathbf{x}} \sim \exp (1)$ for Rayleigh fading \\
$\alpha$ & Path-loss exponent for all the wireless links \\
$P ; r_{0}$ & Transmit power; serving distance for the link of interest \\
\hline \hline
\end{tabular}

$\mathbf{x}$ and $\mathbf{y}$, we consider a standard power law path-loss $l(\mathbf{x}-\mathbf{y})=\|\mathbf{x}-\mathbf{y}\|^{-\alpha}$ with path-loss exponent $\alpha>2$. All the wireless links are assumed to experience independent Rayleigh fading. All the transmitters are assumed to transmit at a fixed power $P$. The received power at the typical node from its transmitter of interest is therefore $P_{r}=P h r_{0}^{-\alpha}$, where $h \sim \exp (1)$ models Rayleigh fading. Similarly, the interference power experienced by the typical receiver located at the origin is

$$
I=\sum_{\mathbf{x} \in \Psi} P h_{\mathbf{x}}\|\mathbf{x}\|^{-\alpha}
$$

where $h_{\mathbf{x}} \sim \exp (1)$ models Rayleigh fading gain for the link from interferer $\mathbf{x} \in \Psi$ to the typical receiver. For this setup, we define coverage probability next.

\subsubsection{SIR and Coverage Probability}

Using the received power over the link of interest and the interference power defined in the previous subsection, the signal to interference ratio (SIR) can 
be expressed as

$$
\operatorname{SIR}\left(r_{0}\right)=\frac{P h r_{0}^{-\alpha}}{\sum_{\mathbf{x} \in \Psi} P h_{\mathbf{x}}\|\mathbf{x}\|^{-\alpha}}
$$

Denote the minimum SIR required for successful decoding and demodulation at the typical receiver by $\gamma$. A useful metric of interest in wireless networks is the SIR coverage probability $\mathrm{P}_{\mathrm{c}}$, which is the probability that the SIR at the receiver exceeds the threshold $\gamma$. Mathematically,

$$
\mathrm{P}_{\mathrm{c}}=\mathbb{P}\left\{\operatorname{SIR}\left(r_{0}\right)>\gamma\right\}=\mathbb{P}\left\{h>\frac{\gamma r_{0}^{\alpha}}{P} I\right\} \stackrel{(a)}{=} \mathbb{E}\left[\exp \left(-\frac{\gamma r_{0}^{\alpha}}{P} I\right)\right] \stackrel{(b)}{=} \mathcal{L}_{I}\left(\frac{\gamma r_{0}^{\alpha}}{P}\right),
$$

where $(a)$ follows from the fact that $h \sim \exp (1)$, and $(b)$ from the definition of Laplace transform of interference power $\mathcal{L}_{I}(s)=\mathbb{E}[\exp (-s I)]$. Note that for this setup, it is sufficient to focus on the Laplace transform of interference in order to study coverage probability. In general, accurate characterization of $\mathcal{L}_{I}(s)$ is the first step in the analysis of more general classes of wireless networks, including cellular networks [73]. Therefore, we will focus on $\mathcal{L}_{I}(s)$ in the technical sections of this chapter with the understanding that the coverage probability can be easily derived for our setup using (3.5). We begin our technical discussion by summarizing two key prior approaches used in the literature for characterizing $\mathcal{L}_{I}(s)$ in the PHP. For the ease of reference, the notation used in this chapter is summarized in Table 3.1. 


\subsection{Key Prior Approaches}

In this section, we summarize two popular approaches that have been used in the literature to derive the Laplace transform of interference in the PHP. At the end of the section, we also provide insights into the strengths and weaknesses of each approach.

\subsubsection{Lower Bound by Ignoring Holes: Approximating $\Psi$ by $\Phi_{2}$}

The first approach is to ignore the effect of holes and approximate the interference field $\Psi$ by the baseline PPP $\Phi_{2}$ of density $\lambda_{2}$. By construction, this approach overestimates the interference power and hence leads to the lower bound on the Laplace transform of interference [30]. This well-known result is stated below for completeness.

Lemma 1 (Lower bound) Ignoring the impact of holes (approximating $\Psi$ by $\left.\Phi_{2}\right)$, the Laplace transform of aggregate interference $I=\sum_{\mathbf{x} \in \Psi} P h_{\mathbf{x}}\|\mathbf{x}\|^{-\alpha}$ is lower bounded by:

$$
\mathcal{L}_{I}(s) \geq \exp \left[-\pi \lambda_{2} \frac{(s P)^{2 / \alpha}}{\operatorname{sinc}(2 / \alpha)}\right]
$$

Proof: $\quad$ See Appendix A.1.

The tightness of the above bound will be demonstrated in the Numerical 
Results section.

\subsubsection{Approximating the PHP by a PPP with the Same Density}

The second approach to the derivation of the Laplace transform of PHP is the first-order statistic approximation [67]. In this approach, the baseline PPP $\Phi_{2}$ is independently thinned such that the resulting density of the PPP is the same as that of the PHP $\Psi$, which we denote by $\lambda_{\mathrm{PHP}}$. The first step in this approach is therefore to derive $\lambda_{\mathrm{PHP}}$ in terms of the given system parameters, which was done in [67]. For completeness, we discuss its proof briefly below. To derive $\lambda_{\mathrm{PHP}}$, we first need to derive an expression for the average number of points of the PHP $\Psi$ lying in a given set $\mathcal{B} \subset \mathbb{R}^{2}$, which by definition is

$$
\begin{aligned}
& \mathbb{E}\left[\sum_{\mathbf{x} \in \Phi_{2} \cap \mathcal{B}} \prod_{\mathbf{y} \in \Phi_{1}}\left(1-\mathbf{1}_{\mathbf{b}(\mathbf{x}, D)}(\mathbf{y})\right)\right] \stackrel{(\mathrm{a})}{=} \mathbb{E}_{\Phi_{2}}\left[\sum_{\mathbf{x} \in \Phi_{2} \cap \mathcal{B}} \mathbb{E}_{\Phi_{1}}\left[\prod_{\mathbf{y} \in \Phi_{1}}\left(1-\mathbf{1}_{\mathbf{b}(\mathbf{x}, D)}(\mathbf{y})\right)\right]\right] \\
& \stackrel{(\mathrm{b})}{=} \mathbb{E}_{\Phi_{2}}\left[\sum_{\mathbf{x} \in \Phi_{2} \cap \mathcal{B}} \exp \left(-\lambda_{1} \int_{\mathbb{R}^{2}} \mathbf{1}_{\mathbf{b}(\mathbf{x}, D)}(\mathbf{y}) \mathrm{d} \mathbf{y}\right)\right] \stackrel{(\mathrm{c})}{=}|\mathcal{B}| \lambda_{2} \exp \left(-\lambda_{1} \pi D^{2}\right),
\end{aligned}
$$

where (a) is due to the independence of point processes $\Phi_{1}$ and $\Phi_{2}$, (b) follows from the probability generating functional (PGFL) of a PPP, and (c) follows from the Campbell theorem [71] in which $|\mathcal{B}|$ denotes the 2-dimensional Lebesgue measure of $\mathcal{B}$. From the above expression, we can readily infer that $\lambda_{\mathrm{PHP}}=\lambda_{2} \exp \left(-\lambda_{1} \pi D^{2}\right)$. Now to derive the Laplace transform of interference 
in this case, we just need to replace $\lambda_{2}$ in the result of Lemma 1 with $\lambda_{\text {PHP }}$. The result is stated below for completeness.

Lemma 2 (Approximation) The Laplace transform of interference power which can be denoted by $I=\sum_{\mathbf{x} \in \Psi} P h_{\mathbf{x}}\|\mathbf{x}\|^{-\alpha}$ when $P H P \Psi$ is approximated by a PPP with density $\lambda_{\mathrm{PHP}}$ is

$$
\mathcal{L}_{I}(s) \simeq \exp \left[-\pi \lambda_{\mathrm{PHP}} \frac{(s P)^{2 / \alpha}}{\operatorname{sinc}(2 / \alpha)}\right]
$$

Remark 1 Both the approaches discussed above approximate $\Psi$ with a homogeneous PPP: the first one with density $\lambda_{2}$ (the baseline PPP), and the second one with density $\lambda_{\mathrm{PHP}}<\lambda_{2}$ (the density of the PHP $\Psi$ ). While the second approach is a seemingly more refined approach, a careful thought reveals that in order to match the density of the PPP with that of the PHP, the baseline PPP has to be independently thinned, which disturbs the local neighborhood of points around the typical node, thus resulting in a loose bound. On the other hand, approximating $\Psi$ simply by the baseline PPP $\Phi_{2}$ preserves the local neighborhood resulting in a relatively tighter approximation. More insights will be provided in the numerical results section.

\subsubsection{Approximating the PHP by a PCP}

There is one more fitting-based approach in which the PHP is approximated by a PCP (such as Thomas or Matérn Cluster Processes) by matching the first 
Chapter 3: Poisson Hole Process: Theory and Applications to WiRELESS NETWORKS

and second order statistics $[30,47]$. The Laplace transform of interference and other performance metrics are then studied using the fitted PCP. As is done in the literature, e.g., [30], we focus on fitting the Thomas cluster process (TCP) in which the number of nodes in each cluster is Poisson distributed with mean $\bar{m}$. The cluster centers (parent points) are drawn from a PPP with density $\lambda_{\mathrm{T}}$ and cluster members are independent and identically distributed (i.i.d.) according to a Gaussian distribution with variance $\sigma^{2}$ in $\mathbb{R}^{2}$ around each cluster center.

The first-order statistic can be used to match the density of the PHP $\left(\lambda_{\mathrm{PHP}}\right)$ with that of a PCP as follows:

$$
\lambda_{2} \exp \left(-\lambda_{1} \pi D^{2}\right)=\lambda_{\mathrm{T}} \bar{m}
$$

where $\bar{m}$ denotes the mean number of points in each cluster. The second-order statistic for a motion invariant point process on $\mathbb{R}^{2}$ can be obtained through pair correlation function [70]. For the TCP, the pair correlation function is

$$
g_{\mathrm{T}}(r)=1+\frac{1}{4 \pi \lambda_{\mathrm{T}} \sigma^{2}} \exp \left(-\frac{r^{2}}{4 \sigma^{2}}\right)
$$

where $r$ denotes the distance between two points of the point process. Since there is no closed-form expression for the pair correlation function of the PHP, we need to obtain it by simulation. Then, we will be able to estimate the parameters of the TCP, $\sigma$ and $\lambda_{\mathrm{T}}$ by using this result [30]. To this end, 
a curve-fitting method like nonlinear least-squares fit (e.g., nlinfit function in Matlab) is used. Substituting $\lambda_{\mathrm{T}}$ back in equation (3.8), we get $\bar{m}$.

We perform analysis for a typical node, which is a randomly chosen node in a randomly chosen cluster, termed as representative cluster. The total interference experienced by the typical node can be written as the sum of two independent terms: intra- and inter-cluster interference caused by the interfering nodes inside the representative cluster, and by the interfering nodes outside the representative cluster. We now characterize the Laplace transform of intra- and inter-cluster interference powers at the typical node in the following Lemmas $[76,77]$. The proofs follow on the same lines as that of [77, Lemmas 2 and 4] and are hence skipped.

Lemma 3 (Laplace transform of intra-cluster interference) For a typical node located at distance $\nu_{0}$ from its cluster center, the Laplace transform of intra-cluster distribution is given by

$$
\mathcal{L}_{I_{\text {intra }}}\left(s \mid \nu_{0}\right)=\exp \left(-\bar{m} \int_{0}^{\infty} \frac{s w^{-\alpha}}{1+s w^{-\alpha}} f_{W}\left(w \mid \nu_{0}\right) \mathrm{d} w\right)
$$

where $w$ and $f_{W}\left(w \mid \nu_{0}\right)=\frac{w}{\sigma^{2}} \exp \left(-\frac{w^{2}+\nu_{0}^{2}}{2 \sigma^{2}}\right) I_{0}\left(\frac{w \nu_{0}}{\sigma^{2}}\right)$ denote the distance from the intra-cluster interferers to the typical node and its PDF, and $I_{0}($.$) is the$ modified Bessel function of the first kind with order zero.

Lemma 4 (Laplace transform of inter-cluster interference) Laplace trans- 
Chapter 3: Poisson Hole Process: Theory and Applications to

form of the inter-cluster interference distribution is given by

$\mathcal{L}_{I_{\text {inter }}}(s)=\exp \left(-2 \pi \lambda_{\mathrm{T}} \int_{0}^{\infty}\left(1-\exp \left(-\bar{m} \int_{0}^{\infty} \frac{s u^{-\alpha}}{1+s u^{-\alpha}} f_{U}(u \mid \nu) \mathrm{d} u\right)\right) v \mathrm{~d} v\right)$

where $u$ and $f_{U}(u \mid \nu)=\frac{u}{\sigma^{2}} \exp \left(-\frac{u^{2}+\nu^{2}}{2 \sigma^{2}}\right) I_{0}\left(\frac{u \nu}{\sigma^{2}}\right)$ denote distance from the inter-cluster interferers to the typical node and its PDF.

Now, using the Laplace transforms of intra- and inter-cluster interference in Lemmas (3) and (4), the Laplace transform of interference for a typical node is derived in the next Theorem.

Theorem 1 (Approximation using PCP) The Laplace transform of interference experienced by a typical node in a TCP is

$$
\mathcal{L}_{I}(s) \simeq \int_{0}^{\infty} \mathcal{L}_{I_{\text {inter }}}(s) \mathcal{L}_{I_{\text {intra }}}\left(s \mid \nu_{0}\right) f_{V_{0}}\left(\nu_{0}\right)
$$

where $f_{V_{0}}\left(\nu_{0}\right)=\frac{\nu_{0}}{\sigma^{2}} \exp \left(-\frac{\nu_{0}^{2}}{2 \sigma^{2}}\right)$ denotes the PDF of distance between the cluster center and the typical node which is a Rayleigh distributed random variable.

Proof: As shown in (3.5), the coverage probability for our setup is the Laplace transform of interference evaluated at $s=\frac{\gamma r_{0}^{\alpha}}{P}$. The result follows from the fact that intra- and inter-cluster interference powers are independent. Then, the resulting approximation from coverage probability is given by deconditioning over $V_{0}$ which denotes the distance from typical node to the cluster center. 


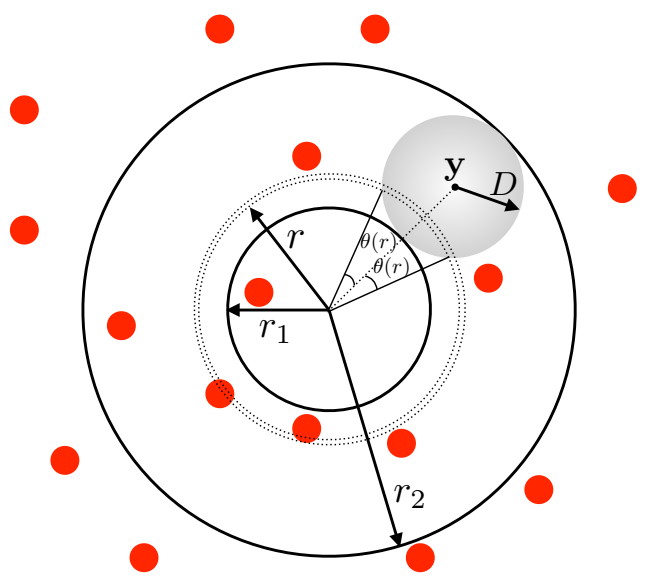

Figure 3.1: Illustration of the interference field with a single hole.

The tightness of the above approximation will be demonstrated in the $\mathrm{Nu}$ merical Results section. In particular, we will show that this approach works better than the two prior approaches. That being said, it should be noted that the higher-order statistics of the PHP and the fitted-PCP may be different, which means that the accuracy of this approximation is a function of system parameters. Furthermore, the resulting expressions for the Laplace transform of interference in the fitted-TCP are complex than the bounds obtained using the proposed approach in the next section. We now discuss the proposed approaches next.

\subsection{Proposed Approaches to Laplace Trans-}

\section{form of Interference in the PHP}

We now introduce our proposed approach to characterize the Laplace transform of interference in the PHP. In the first intermediate step, we model the 
Chapter 3: Poisson Hole Process: Theory and Applications to WIRELESS NETWORKS

locations of interferers by a homogeneous PPP $\Phi_{2}$ of density $\lambda_{2}$ from which only one hole $\mathcal{C}$ of radius $D$ is carved out at a deterministic location. Let the location of the center of this hole be $\mathbf{y} \in \mathbb{R}$ and hence its distance from the origin be $\|\mathbf{y}\|$. The resulting setup is illustrated in Fig. 3.1. Here, solid red dots represent the locations of interferers.

Note that the interference field in this case is non-isotropic due to the fixed location of the hole. The Laplace transform of the interference power at the origin from the nodes of $\Phi_{2}$ outside $\mathcal{C}$ is characterized next. This intermediate result will be used later in this chapter to derive upper and lower bounds on the Laplace transform of interference experienced by a typical node in the PHP.

Lemma 5 Let $I=\sum_{\mathbf{x} \in \Phi_{2} \cap \mathbf{b}^{c}(\mathbf{y}, D)} P h_{\mathbf{x}}\|\mathbf{x}\|^{-\alpha}$, the Laplace transform of interference conditioned on $\|\mathbf{y}\|$ is

$$
\mathcal{L}_{I \mid\|\mathbf{y}\|}(s)=\exp \left(-\pi \lambda_{2} \frac{(s P)^{2 / \alpha}}{\operatorname{sinc}(2 / \alpha)}\right) \exp \left(\int_{\|\mathbf{y}\|-D}^{\|\mathbf{y}\|+D} \frac{2 \pi \lambda(r)}{1+\frac{r^{\alpha}}{P s}} r \mathrm{~d} r\right)
$$

where $\lambda(r)=\frac{\lambda_{2}}{\pi} \arccos \left(\frac{r^{2}+\|\mathbf{y}\|^{2}-D^{2}}{2\|\mathbf{y}\| r}\right), \mathbf{b}(\mathbf{y}, D)$ denotes the hole centered at $\mathbf{y}$ with radius $D$.

Proof: See Appendix A.2.

Remark 2 (Dissolving the hole) The above result has an interesting interpretation that will be useful in visualizing the proposed results. Note that 
since received power is a radially symmetric function, it solely depends upon the distance of the transmitter to the origin. In other words, if we consider interfering nodes located in a thin strip of radius $\|\mathbf{y}\|-D \leq r \leq\|\mathbf{y}\|+D$ and vanishingly small width $\mathrm{d} r$, the angular distribution of these nodes does not affect the interference observed at the typical node. Therefore, we can, in principle, "dissolve the hole" as long as the number of points lying in this thin strip is not changed.

Please refer to Fig. 1 for an illustration of this strip. Taking a closer look at the interference originating from this strip, we note that the only thing that matters is the number of points distributed in the part of the strip which is outside the hole. The area of this region is $2 r \mathrm{~d} r(\pi-\theta(r))$, where the angle $\theta(r)=\arccos \left(\frac{r^{2}+\|\mathbf{y}\|^{2}-D^{2}}{2\|\mathbf{y}\| r}\right)$ is defined in Fig. 1. Therefore, the number of interfering points lying within this strip is Poisson distributed with mean $\lambda_{2} 2 r \mathrm{~d} r(\pi-\theta(r))$. Since the exact locations of these points within the strip does not matter, we can dissolve the hole and redistribute the points uniformly inside the whole strip of area $2 \pi r \mathrm{~d} r$. This means, the PPP with a hole can be equivalently modeled as a non-homogeneous PPP with density $\lambda_{2}(1-\theta(r) / \pi)$, where the $\lambda_{2} \theta(r) / \pi$ term (defined as $\lambda(r)$ in Lemma 5) captures the effect of hole.

Using this intermediate result and the above insights, we now derive tight bounds on the Laplace transform of interference experienced by a typical node in the PHP. 


\subsubsection{Lower Bound on the Laplace Transform of Inter-}

\section{ference in the PHP}

Before going into the technical details, note that due to path-loss, the effect of holes that are close to the typical node will be much more significant compared to the holes that are farther away. Therefore, to derive an easy-touse lower bound on the Laplace transform of interference, we consider only one hole; the one that is closest to the typical node; and ignore the other holes. Denoting the location of the closest hole by $\mathbf{y}_{1}$, the interference field in this case is $\left(\Phi_{2} \cap \mathbf{b}^{c}\left(\mathbf{y}_{1}, D\right)\right) \supset \Psi$, which clearly overestimates the interference of the PHP and hence leads to a lower bound on the Laplace transform. Note that in Lemma 5, we have already derived the conditional Laplace transform for the case when there is one hole and its distance to the origin is known. To derive a lower bound, we simply need to assume this hole to be the closest point of $\Phi_{1}$ to the origin and decondition the result of Lemma 5 with respect to the distribution of $V_{1}=\left\|\mathbf{y}_{1}\right\|$. To this end, we first derive the probability density function (PDF) of $V_{1}$ next.

Lemma 6 The PDF of the distance $V_{1}=\left\|\mathbf{y}_{1}\right\|$ between the typical node at the origin and the closest point of $\Phi_{1}$ is given by

$$
f_{V_{1}}\left(v_{1}\right)=2 \pi \lambda_{1} v_{1} \exp \left(-\pi \lambda_{1}\left(v_{1}^{2}-D^{2}\right)\right), \quad v_{1} \geq D
$$

Proof: The typical node of the PHP lies outside the holes by construction. 


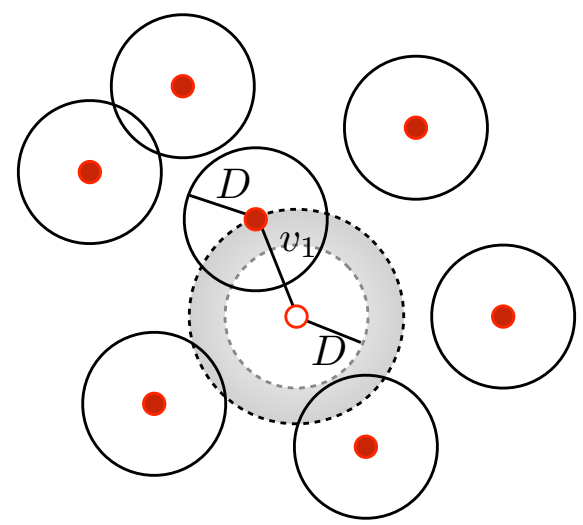

Figure 3.2: Illustration showing that the closest point of $\Phi_{1}$ is at least a distance $D$ away from the typical node of the PHP $\Psi$.

Therefore, as illustrated in Fig. 3.2, the minimum distance between the typical node and the closest hole (closest point of $\Phi_{1}$ ) is D. The white dot and solid red dots represent the typical node of the PHP and points of $\Phi_{1}$, respectively as shown in Fig. 3.2. Using this fact along with the properties of a PPP, the distribution of $V_{1}$ can be derived as follows:

$$
\begin{aligned}
\mathbb{P}\left(V_{1}>v_{1}\right) & =\mathbb{P}\left(\text { Number of points of } \Phi_{1} \text { in the set }\left\{\mathbf{b}\left(0, v_{1}\right) \backslash \mathbf{b}(0, D)\right\}=0\right) \\
& =\exp \left(-\pi \lambda_{1}\left(v_{1}^{2}-D^{2}\right)\right), \quad v_{1} \geq D
\end{aligned}
$$

The result now follows by differentiating the above expression.

Deconditioning the result of Lemma 5 with respect to the distribution of the distance to the closest hole derived above, the proposed lower bound is derived below.

Theorem 2 (New Lower Bound 1) Let $I=\sum_{\mathbf{x} \in \psi} P h_{\mathbf{x}}\|\mathbf{x}\|^{-\alpha}$, the Laplace 
Chapter 3: Poisson Hole Process: Theory and Applications to WIRELESS NETWORKS

transform of interference is lower bounded by

$\mathcal{L}_{I}(s)$

$\geq \exp \left(-\pi \lambda_{2} \frac{(s P)^{2 / \alpha}}{\operatorname{sinc}(2 / \alpha)}\right) \times \int_{D}^{\infty} \exp \left(f\left(v_{1}\right)\right) 2 \pi \lambda_{1} v_{1} \exp \left(-\pi \lambda_{1}\left(v_{1}^{2}-D^{2}\right)\right) \mathrm{d} v_{1}$,

where $f\left(v_{1}\right)=\int_{v_{1}-D}^{v_{1}+D} \arccos \left(\frac{r^{2}+v_{1}^{2}-D^{2}}{2 v_{1} r}\right) \frac{2 \lambda_{2}}{1+\frac{r^{\alpha}}{P s}} r \mathrm{~d} r$.

Proof: See Appendix A.3.

Remark 3 Since the PHP is approximated by $\Phi_{2} \cap \mathbf{b}^{c}\left(\mathbf{y}_{1}, D\right)$ in the above result, the resulting lower bound presented in Theorem 2 is by construction tighter than the known lower bound presented in Lemma 1 where the interference field was approximated by simply $\Phi_{2}$. On the same lines as discussed for Lemma 1 in Remark 1, the above approach captures the local neighborhood of the typical node accurately, thus leading to a remarkably tight lower bound in Theorem 2. This will be demonstrated later in this chapter and in the numerical results section.

\subsubsection{Upper Bound for the Laplace Transform of Inter- ference in the PHP}

To derive an upper bound on the Laplace transform, we extend the above approach to all the holes. To maintain tractability, each hole is carved out 
individually/separately from the baseline PPP $\Phi_{2}$ using the above approach. Note that since the centers of the holes follow a $\operatorname{PPP} \Phi_{1}$, there will obviously be overlaps among holes. Therefore, when we remove points of $\Phi_{2}$ corresponding to each hole individually (without accounting for the overlaps), we may remove certain points multiple times thus underestimating the interference field, which results in an upper bound on the Laplace transform of interference. In the next section, we present several ways to incorporate the effect of overlaps but they are either too complex and hence less tractable or too simple and hence less accurate. Fortunately, the bounds derived in this chapter without incorporating the effect of overlaps are remarkably tight and can be considered proxies for the exact Laplace transform.

Theorem 3 (New Upper Bound) The Laplace transform of interference experienced by a typical node in the PHP is upper bounded by

$$
\mathcal{L}_{I}(s) \leq \exp \left(-\pi \lambda_{2} \frac{(s P)^{2 / \alpha}}{\operatorname{sinc}(2 / \alpha)}\right) \times \exp \left(-2 \pi \lambda_{1}\left(\int_{D}^{\infty}(1-\exp (f(v))) v \mathrm{~d} v\right)\right)
$$

where $f(v)=\int_{v-D}^{v+D} \arccos \left(\frac{r^{2}+v^{2}-D^{2}}{2 v r}\right) \frac{2 \lambda_{2}}{1+\frac{r^{\alpha}}{P s}} r \mathrm{~d} r$.

Proof: See Appendix A.4.

For the same reason as the tightness of lower bound of Theorem 2 discussed in Remark 3, the above upper bound is also remarkably tight for a wide variety of scenarios. More details on the tightness are provided in the next subsection 


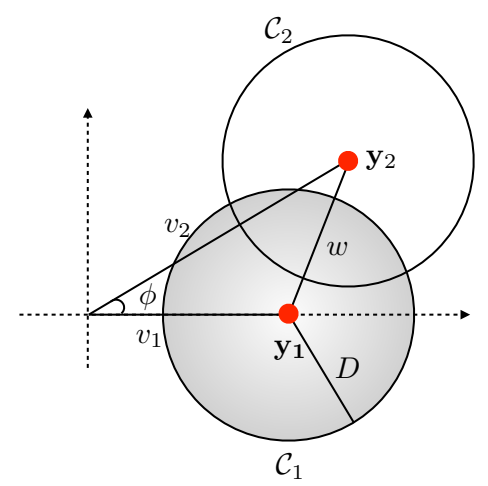

Figure 3.3: Illustration of the setup used in Theorem 4 where only two holes closest to the typical node are considered.

and in the numerical results section.

One way to compare the tightness of the proposed upper and lower bounds is to evaluate their ratio. For instance, if the ratio is close to one, we can infer that both upper and lower bounds are close to the actual simulation values. This ratio will be shown to be close to one across wide range of parameters in the numerical results section. Next, we explore a few ways to incorporate the effect of overlaps in the holes that was ignored in the results derived in this section.

\subsection{Incorporating the Impact of Overlaps in the Proposed Approaches}

The key lower and upper bounds reported in Theorems 2 and 3 in the previous section carefully circumvented the need for considering the overlaps in the holes explicitly. This was to maintain tractability. However, it is quite 
natural to wonder how much tractability is really lost if we try to incorporate the effect of the overlaps in the holes accurately. In this section, we address this question by deriving three results for the Laplace transform of interference that incorporate the effect of holes. In the first result, we generalize the lower bound derived in Theorem 2 by considering two nearest holes instead of a single hole. The overlap among the two holes is explicitly incorporated in the analysis. The setup is presented in Fig. 3.3, where $\mathcal{C}_{1}=\mathbf{b}\left(\mathbf{y}_{1}, D\right)$ and $\mathcal{C}_{2}=\mathbf{b}\left(\mathbf{y}_{2}, D\right)$ denote the first and the second closest holes to the typical receiver, respectively. The angle between $\mathbf{y}_{1}$ and $\mathbf{y}_{2}$ is denoted by $\phi$. The interference field in this case is modeled by $\left(\Phi_{2} \cap\left\{\mathcal{C}_{1} \cup \mathcal{C}_{2}\right\}^{c}\right) \supset \Psi$, which overestimates the interference power and hence leads to a lower bound on the Laplace transform of interference. Before going into the main result, we first need to evaluate the joint PDF of the distances between the first and second closest holes to the origin, which are denoted by random variables $V_{1}$ and $V_{2}$, respectively. Using the same arguments as in Lemma 6, the joint PDF can be derived as $[78]$

$$
f_{V_{1} V_{2}}\left(v_{1}, v_{2}\right)=f_{V_{2}}\left(v_{2} \mid v_{1}\right) f_{V_{1}}\left(v_{1}\right)=\left(2 \pi \lambda_{1}\right)^{2} v_{1} v_{2} \exp \left(-\pi \lambda_{1}\left(v_{2}^{2}-D^{2}\right)\right) .
$$

Using this distribution, the Laplace transform of interference for this case is derived next.

Theorem 4 (New Lower Bound 2) The Laplace transform of interference 


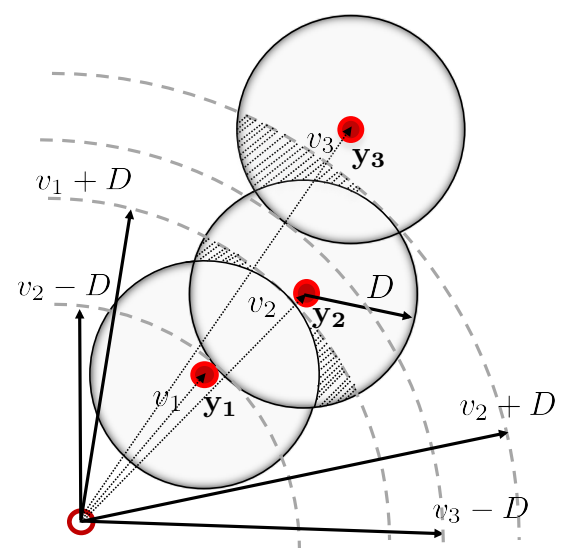

Figure 3.4: Illustration of the setup used in Theorem 5 where $k$ holes closest to the typical node are considered.

experienced by a typical node of the PHP $\Psi$ is lower bounded by

$$
\begin{aligned}
& \mathcal{L}_{I}(s) \geq \exp \left(-\pi \lambda_{2} \frac{(s P)^{2 / \alpha}}{\operatorname{sinc}(2 / \alpha)}\right) \times\left(\frac{1}{2 \pi} \int_{D}^{\infty} \int_{v_{1}}^{\infty} \int_{-\pi}^{\pi} \exp \left(\int_{v_{1}-D}^{v_{1}+D_{2}} \frac{2 \pi \lambda_{c 1}(r)}{1+\frac{r^{\alpha}}{s P}} r \mathrm{~d} r\right)\right. \\
& \left.\times \exp \left(\int_{v_{2}-D}^{v_{2}+D} \frac{2 \pi \lambda_{c 2}(r)}{1+\frac{r^{\alpha}}{s P}} r \mathrm{~d} r\right) \exp \left(-\lambda_{2} \mathcal{B}\left(v_{1}, v_{2}, \phi\right)\right) \times f_{V_{1} V_{2}}\left(v_{1}, v_{2}\right) \mathrm{d} \phi \mathrm{d} v_{2} \mathrm{~d} v_{1}\right)
\end{aligned}
$$

where $\lambda_{c i}(r)=\frac{\lambda_{2}}{\pi} \arccos \left(\frac{r^{2}+v_{i}{ }^{2}-D^{2}}{2 v_{i} r}\right)$, for $i=1,2$ and $\mathcal{B}\left(v_{1}, v_{2}, \phi\right)$ is given by (A.9).

Proof: The key idea behind this proof is to consider $\left(\Phi_{2} \cap\left\{\mathbf{b}\left(\mathbf{y}_{1}, D\right) \cup\right.\right.$ $\left.\left.\mathbf{b}\left(\mathbf{y}_{2}, D\right)\right\}^{c}\right) \supset \Psi$ as the interference field, which clearly overestimates the interference and hence leads to the lower bound. Complete proof is provided in Appendix A.5.

As evident from the above result, incorporating the effect of overlap, even among two holes, results in a significantly more complex expression compared 
to the bounds presented in Theorems 2 and 3. This shows that incorporating the exact effect of overlaps does indeed lead to a significant loss in tractability. Therefore, instead of trying to incorporate the exact effect of overlaps, we now propose a new procedure for bounding the overlap area, which allows us to derive a lower bound on the Laplace transform while considering multiple holes in the interference field. This tightens the result of Theorem 2, where only one hole was considered. In particular, we consider $k$ closest holes from the typical receiver, as shown in Fig. 3.4. In order to claim the result as a bound, we bound the union of $k$-closest holes, i.e., $\cup_{i=1}^{k} \mathcal{C}_{i}=\cup_{i=1}^{k} \mathbf{b}\left(\mathbf{y}_{i}, D\right)$, with $\Omega_{\mathrm{o}}=$ $\mathcal{C}_{1} \cup_{i=2}^{k}\left\{\mathbf{b}\left(\mathbf{y}_{i}, D\right) \bigcap \mathbf{b}^{c}\left(0, \max \left(\left\|\mathbf{y}_{i-1}\right\|+D,\left\|\mathbf{y}_{i}\right\|-D\right)\right\}\right.$, where $\Omega_{\mathrm{o}} \subset \cup_{i=1}^{k} \mathcal{C}_{i}$. The set $\Omega_{\mathrm{o}}$ carefully avoids the overlapping part and hence does not result in over-removal of the points from the baseline point process $\Phi_{2}$. The contribution of $i^{t h}$ hole in $\Omega_{\mathrm{o}}$ is

$$
\mathbf{d}\left(\mathbf{y}_{i}, D\right)=\left\{\mathbf{b}\left(\mathbf{y}_{i}, D\right) \bigcap \mathbf{b}^{c}\left(0, \max \left(\left\|\mathbf{y}_{i-1}\right\|+D,\left\|\mathbf{y}_{i}\right\|-D\right)\right\}\right.
$$

In Fig. 3.4, the set $\mathbf{d}\left(\mathbf{y}_{i}, D\right)$ corresponds to the unshaded part of a hole that does not overlap with the other holes. Clearly, this approach results in the removal of fewer points from the baseline process $\Phi_{2}$ compared to the PHP, which leads to a lower bound on the Laplace transform of interference in the PHP. This lower bound is presented in the next theorem.

Theorem 5 (New Lower Bound 3) The Laplace transform of interference 
is bounded by

$$
\begin{aligned}
& \mathcal{L}_{I}(s) \geq \\
& \exp \left(-\pi \lambda_{2} \frac{(s P)^{2 / \alpha}}{\sin (2 / \alpha)}\right) \times\left(\iiint_{D<v_{1}<v_{2}<\ldots<v_{k}<\infty} \exp \left(\int_{v_{1}-D}^{v_{1}+D} \frac{2 \pi \lambda_{c 1}(r)}{1+\frac{r^{\alpha}}{s P}} r \mathrm{~d} r\right)\right. \\
& \times \exp \left(\int_{\max \left(v_{2}-D, v_{1}+D\right)}^{v_{2}+D} \frac{2 \pi \lambda_{c 2}(r)}{1+\frac{r^{\alpha}}{s P}} r \mathrm{~d} r\right) \ldots \exp \left(\int_{\max \left(v_{k}-D, v_{k-1}+D\right)}^{v_{k}+D} \frac{2 \pi \lambda_{c k}(r)}{1+\frac{r^{\alpha}}{s P}} r \mathrm{~d} r\right) \\
& \left.\times f_{V_{1} V_{2} . . V_{k}}\left(v_{1}, v_{2}, . ., v_{k}\right) \mathrm{d} v_{1} \mathrm{~d} v_{2} \ldots \mathrm{d} v_{k}\right)
\end{aligned}
$$

where $\lambda_{\mathrm{c} i}(r)=\frac{\lambda_{2}}{\pi} \arccos \left(\frac{r^{2}+v_{i}{ }^{2}-D^{2}}{2 v_{i} r}\right)$ for $i=1,2, \ldots, k$, and joint density function of distances of $V_{1}, V_{2}, \ldots, V_{k}$ is $f_{V_{1} V_{2} . . V_{k}}\left(v_{1}, v_{2}, . ., v_{k}\right)=\left(2 \pi \lambda_{1}\right)^{k} v_{1} v_{2} \ldots v_{k}$ $\times \exp \left(-\pi \lambda_{1}\left(v_{k}^{2}-D^{2}\right)\right)$.

Proof: See Appendix A.6.

In the numerical results section, we will show that considering $k=2$, i.e., only the two closest holes from the typical node, results in a remarkably tight bound. Note that for $k=2$, the expression is also fairly tractable. For brevity, that expression is not stated separately.

Now, in Theorems 4 and 5, we have tried to handle the overlaps in such a way that the resulting expressions: (i) can be claimed as lower bounds to the Laplace transform, and (ii) tighten the lower bound provided by Theorem 2 . One last question that we address before concluding this section is whether it is possible to handle the effect of overlaps in the average sense. For this, we revisit the upper bound of Theorem 3, which was derived by carving out holes 
from the baseline process $\Phi_{2}$ individually without caring about the overlaps between them. This led to the possible removal of some points multiple times, thereby leading to an underestimation of interference. We compensate this over-removal, by rescaling the second term of Theorem 3, which is the one that captures the effect of removing points from $\Phi_{2}$. The rescaling term is derived by estimating the average pairwise overlap area between circles. More details of the approach are provided in the proof in Appendix A.7. Note that the resulting expression in this case is the same as that of Theorem 3, except a scaling factor of $1-\min \left(\frac{\lambda_{1} \pi D^{2}}{2}, \frac{1}{2}\right)$ that appears in the second term. The key downside to this approach compared to Theorem 3 is that it results in an approximation unlike Theorem 3, where the result was shown to be a bound.

Proposition 1 (New Approximation) The Laplace transform of interference at a typical node in the PHP can be approximated as

$$
\begin{aligned}
\mathcal{L}_{I}(s) \simeq & \exp \left(-\pi \lambda_{2} \frac{(s P)^{2 / \alpha}}{\operatorname{sinc}(2 / \alpha)}\right) \\
& \times \exp \left[-2 \pi \lambda_{1} \int_{D}^{\infty}\left(1-\exp \left(f(v)\left(1-\min \left(\frac{\lambda_{1} \pi D^{2}}{2}, \frac{1}{2}\right)\right)\right)\right) v \mathrm{~d} v\right]
\end{aligned}
$$

where $f(v)=\int_{v-D}^{v+D} \arccos \left(\frac{r^{2}+v^{2}-D^{2}}{2 v r}\right) \frac{2 \lambda_{2}}{1+\frac{r \alpha}{P s}} r \mathrm{~d} r$.

Proof: See Appendix A.7.

Comparison of this result with the bounds derived in this section shows that 
Chapter 3: Poisson Hole Process: Theory and Applications to WiRELESS NETWORKS

handling overlaps in the average sense may not work particularly well, especially when the overlaps are significant. This is because such results do not capture the local neighborhood of the typical node as carefully as the bounds derived in this section. We now move on the numerical results section, where more such insights about the relative accuracy of bounds and approximations are presented.

\subsection{Numerical Results and Discussion}

There are three main system parameters that determine the interference experienced by the typical node in the PHP: density $\lambda_{1}$ of the holes, density $\lambda_{2}$ of the baseline PPP, and the radii $D$ of the holes. Based on the relative values of these parameters, we identify four main network configurations, which are illustrated in Fig. 3.5. We define the possible configurations as LD-SH: configuration with low density of holes and small holes; HD-SH: configuration with high density of holes and small holes; LD-LH: configuration with low density of holes and large holes; HD-LH: configuration with high density of holes and large holes. Clearly, the configuration where the holes are small and sparse (LD-SH case) is more benign than the configuration in which the holes are small and dense (HD-SH). Similarly, the configuration where holes are large and sparse (LD-LH case) is more benign than the configuration where the holes are both large and dense (HD-LH case). Therefore, 


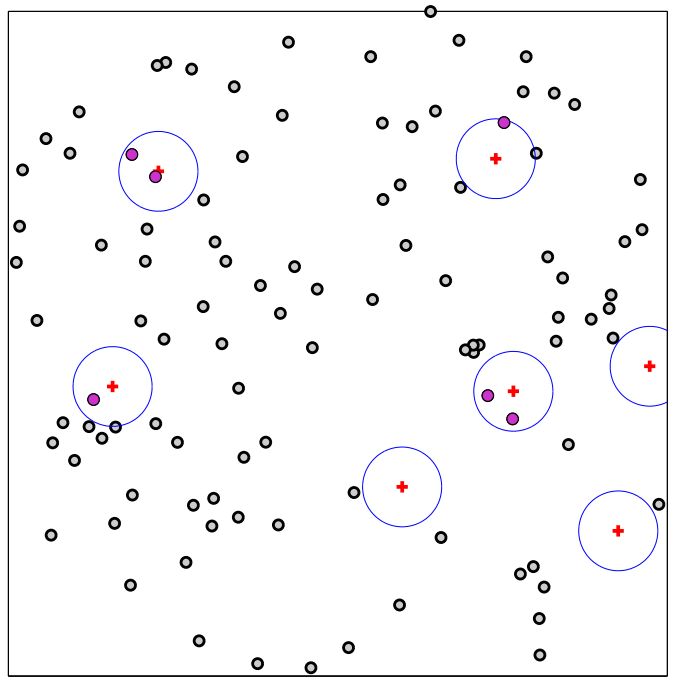

(a)

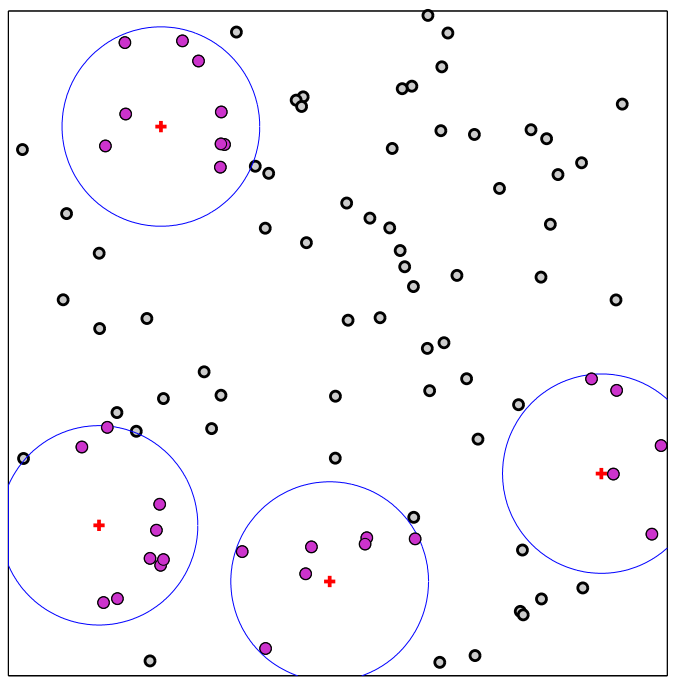

(c)

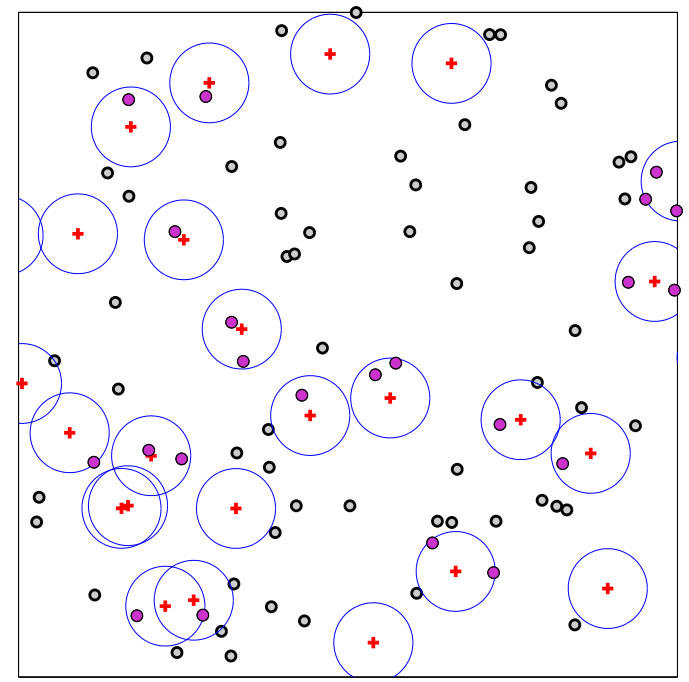

(b)

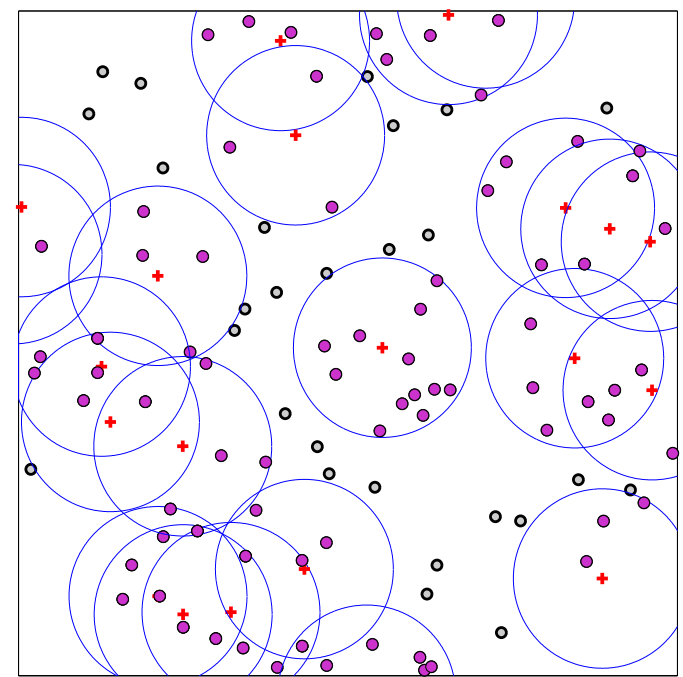

(d)

Figure 3.5: The PHP network model (a) First configuration: LD-SH, (b) Second configuration: HD-SH, (c) Third configuration: LD-LH, (d) Fourth configuration: HD-LH.

the result that works well in the HD-SH configuration is expected to work in the LD-SH configuration as well. The same is true for LD-LH and HD-LH cases. Simulations are performed over circular region with radius $40 \mathrm{~m}$ and results are averaged over $5 \times 10^{4}$ iterations. Unless otherwise specified, we set 


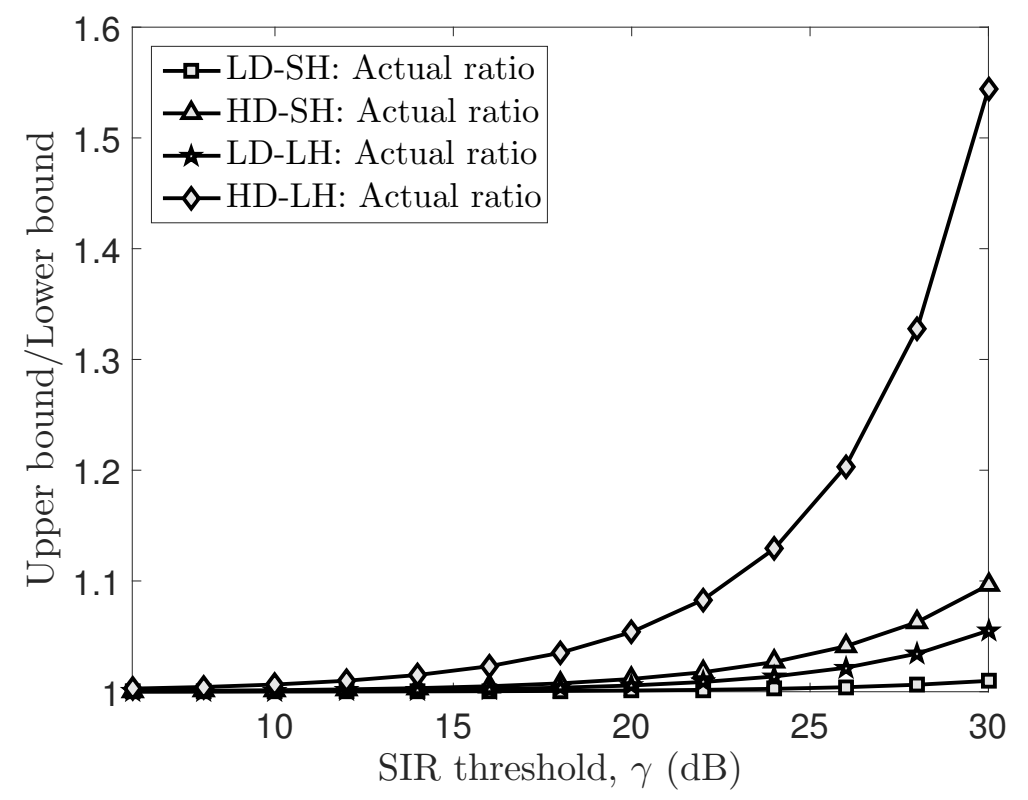

Figure 3.6: Ratio of the proposed upper and lower bounds derived in Theorems 3 and 2, respectively.

the network parameters as follows: $\lambda_{2}=1, \alpha=4, P=1, r_{0}=0.1, \gamma=10$ dB. This choice of the simulation setup, especially density $\lambda_{2}$ and radius of the simulation region, ensures that the border effects due to the finite simulation area are minimized. We compare our proposed bounds and the new approximation with the first-order statistic approximation given by Lemma 2, and the PPP-based bound given by Lemma 1 where $\Psi$ is approximated by $\Phi_{2}$. Before going into more details comparisons, we demonstrate the tightness of the lower and upper bounds derived in Theorems 2 and 3 by plotting their ratio in Fig. 3.6. This result shows that the ratio in all cases of interest is close to one, which demonstrates the tightness of both the bounds. Note that, as expected, the ratio is comparatively higher when the holes are large and dense (HD-LH case). This is because in this case, increasing $\gamma$ results in a 


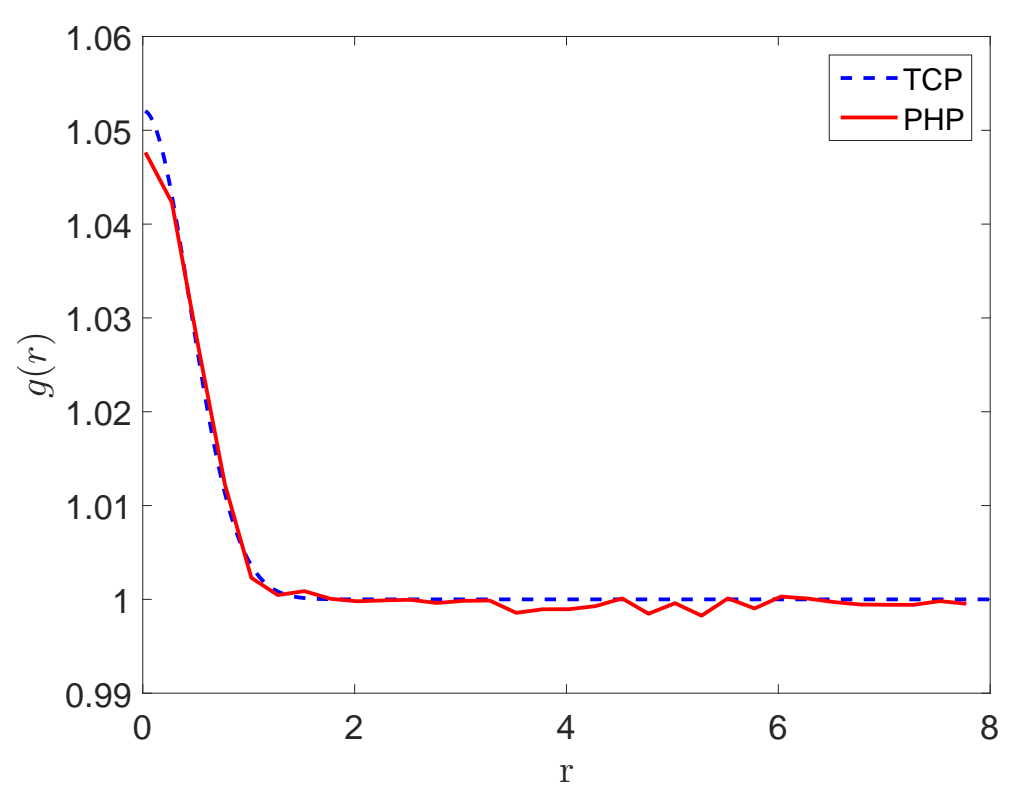

Figure 3.7: Comparison of g-functions of the Poisson hole process and the Thomas cluster process in LD-SH case $\left(\lambda_{1}=0.05\right.$ and $\left.D=0.6\right)$.

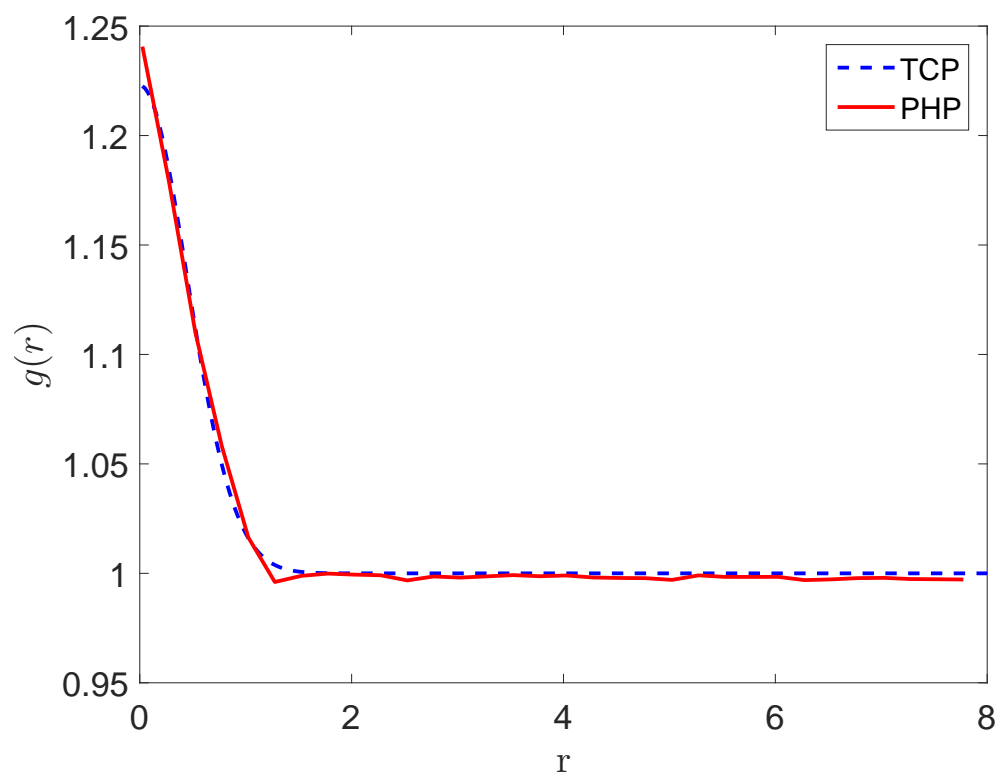

Figure 3.8: Comparison of g-functions of the Poisson hole process and the Thomas cluster process in HD-SH case $\left(\lambda_{1}=0.2\right.$ and $\left.D=0.6\right)$.

relatively loose upper bound.

We now compare the proposed bounds and approximations with the nu- 
Chapter 3: Poisson Hole Process: Theory and Applications to WiRELESS NETWORKS

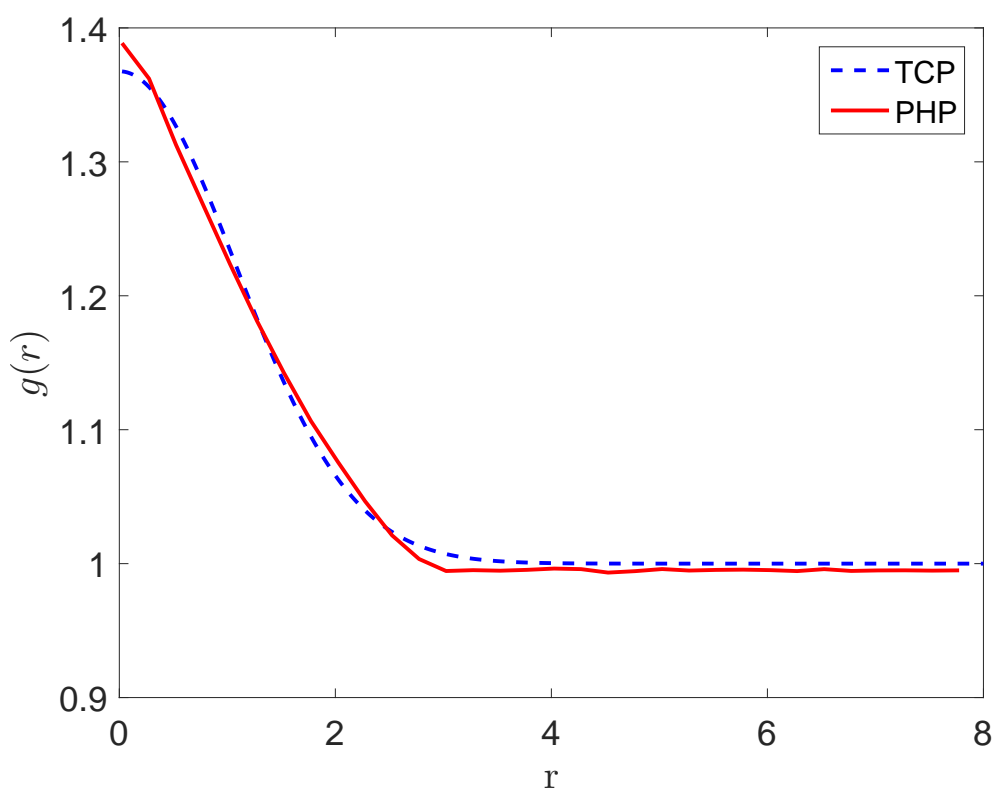

Figure 3.9: Comparison of g-functions of the Poisson hole process and the Thomas cluster process in LD-LH case $\left(\lambda_{1}=0.05\right.$ and $\left.D=1.5\right)$.

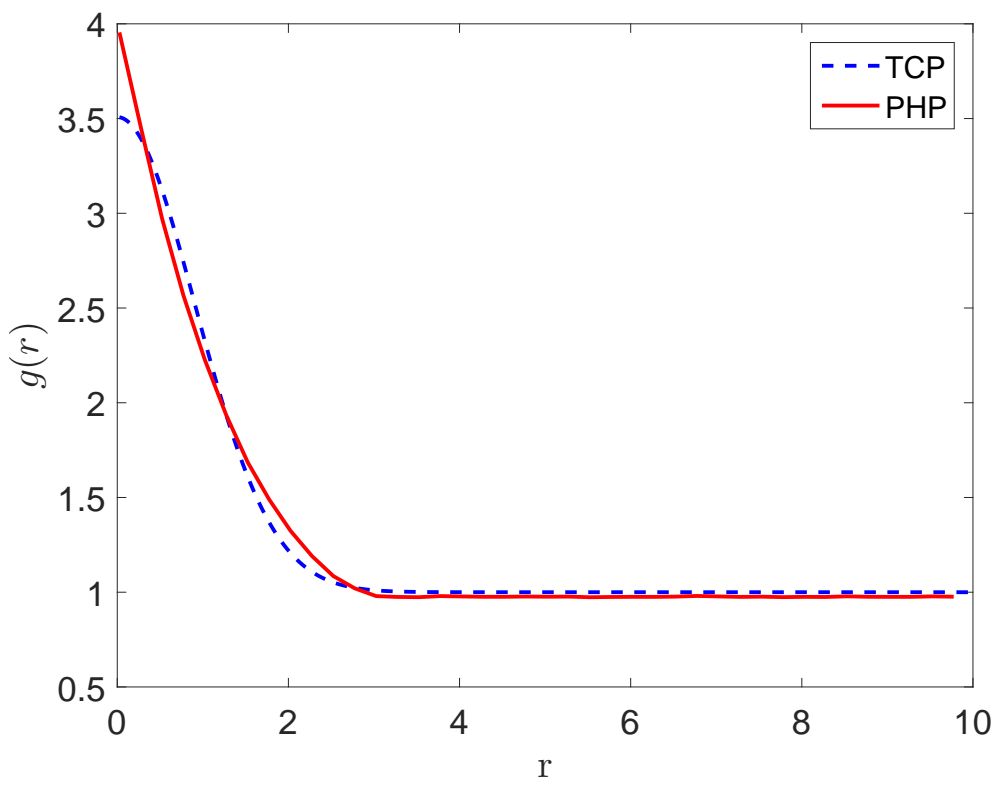

Figure 3.10: Comparison of g-functions of the Poisson hole process and the Thomas cluster process in HD-LH case $\left(\lambda_{1}=0.2\right.$ and $\left.D=1.5\right)$.

merical results and the known approaches in terms of coverage probability. For the comparison of the PHP model with the PCP model, as discussed in 
section 3.3.3, the parameters of the TCP can be determined by using curvefitting and matching the first and second order statistics. We now plot the g-functions of the PHP and TCP obtained by simulation in Figs. 3.7- 3.10. Recall that the g-function is the pair correlation function, as discussed in section 3.3.3. The results show that the second order density of PHP can be approximated fairly accurately by a TCP. We have shown the Laplace transform results given by the TCP approximation in Figs. 3.13-3.16 which are denoted by the legend Prior approximation 2: Theorem 1. This approximation works well compared to the prior lower bound based on ignoring the effect of the holes, and prior approximation, which is based on matching the PPP density to that of a PHP. However, this approach has a few shortcomings. First, it is a curve-fitting based approach in which we need to determine fitting parameters for the PCP for each configuration of the PHP. In other words, since there is no closed form mathematical expression for the pair correlation function of the PHP, the second order estimation of the PCP is obtained by simulating the pair correlation function of the PHP and then by using curve-fitting methods, which make this approach more complicated. Second, the resulting Laplace transform expressions are also more complex compared to the ones obtained by our proposed approach for the analysis of PHP. In general, the proposed bounds are also more accurate than the PCP based approximations.

As demonstrated in (3.5), the coverage probability for our setup is simply the Laplace transform of interference evaluated at $s=\frac{\gamma r_{0}^{\alpha}}{P}$. Note that when we 


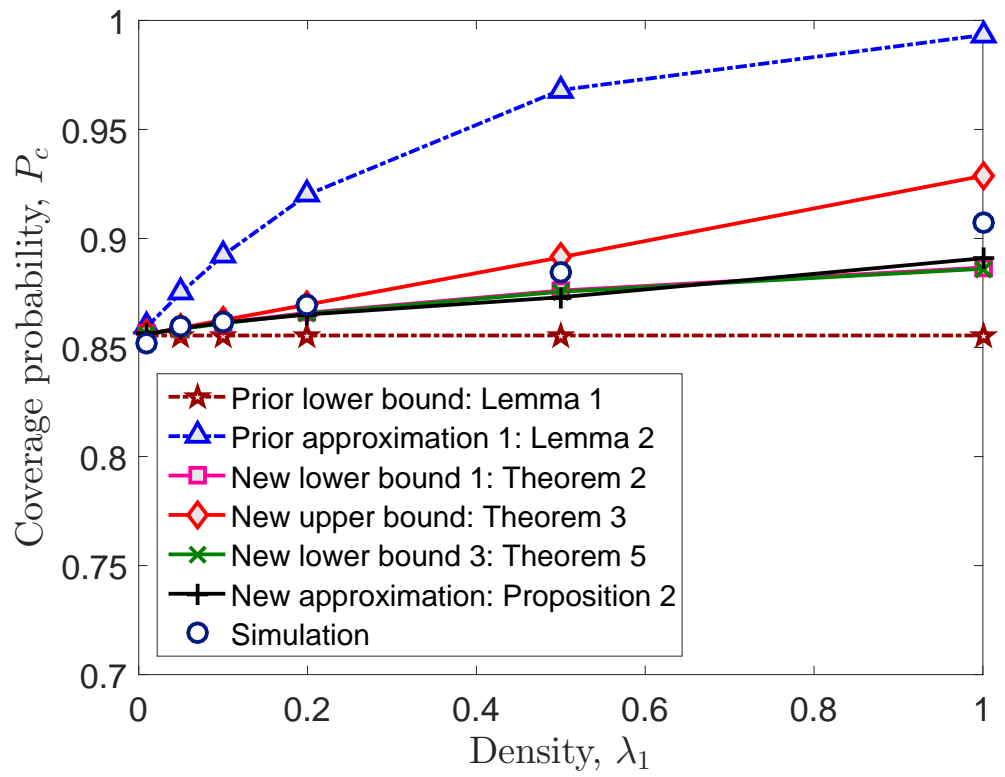

Figure 3.11: Analytical and simulation results for the coverage probability as a function of the density $\lambda_{1}(D=1)$.

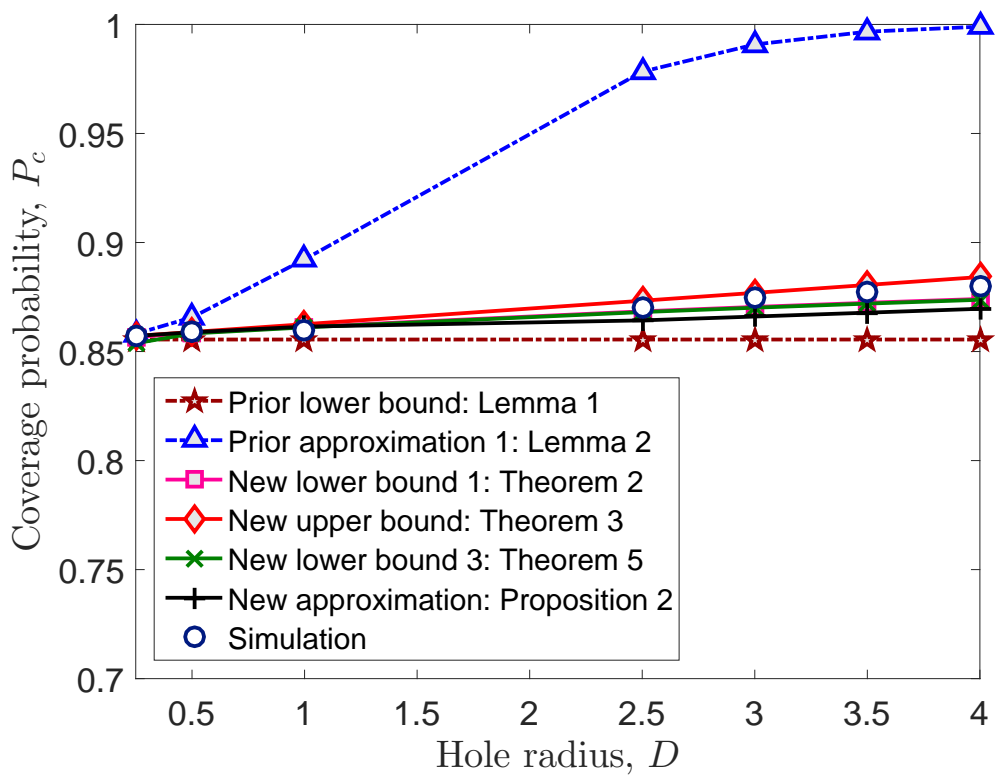

Figure 3.12: Analytical and simulation results for the coverage probability as a function of the hole radius $D\left(\lambda_{1}=0.1\right)$.

substitute $s=\frac{\gamma r_{0}^{\alpha}}{P}$ in any of the Laplace transform expressions derived in this chapter, we notice that the resulting expression is independent of $P$. This is expected, because the $\operatorname{SIR}\left(r_{0}\right)$ expression given by (5.2) is indeed independent 


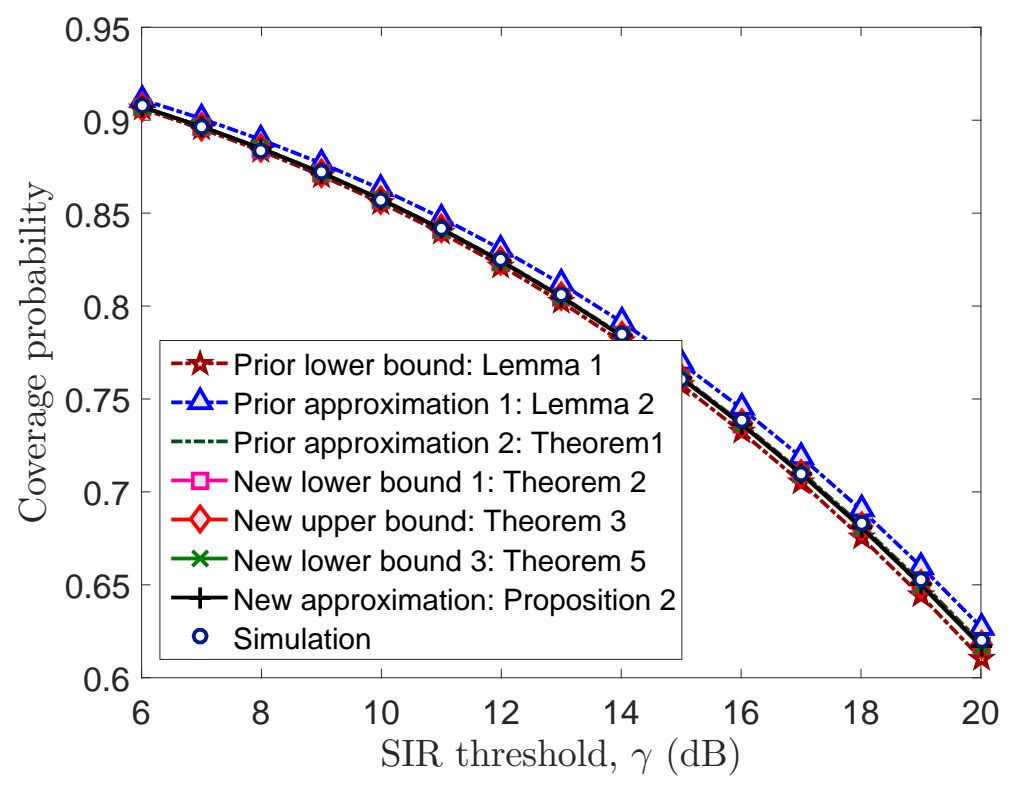

Figure 3.13: Analytical and simulation results for the coverage probability in LD-SH case $\left(\lambda_{1}=0.05\right.$ and $\left.D=0.6\right)$.

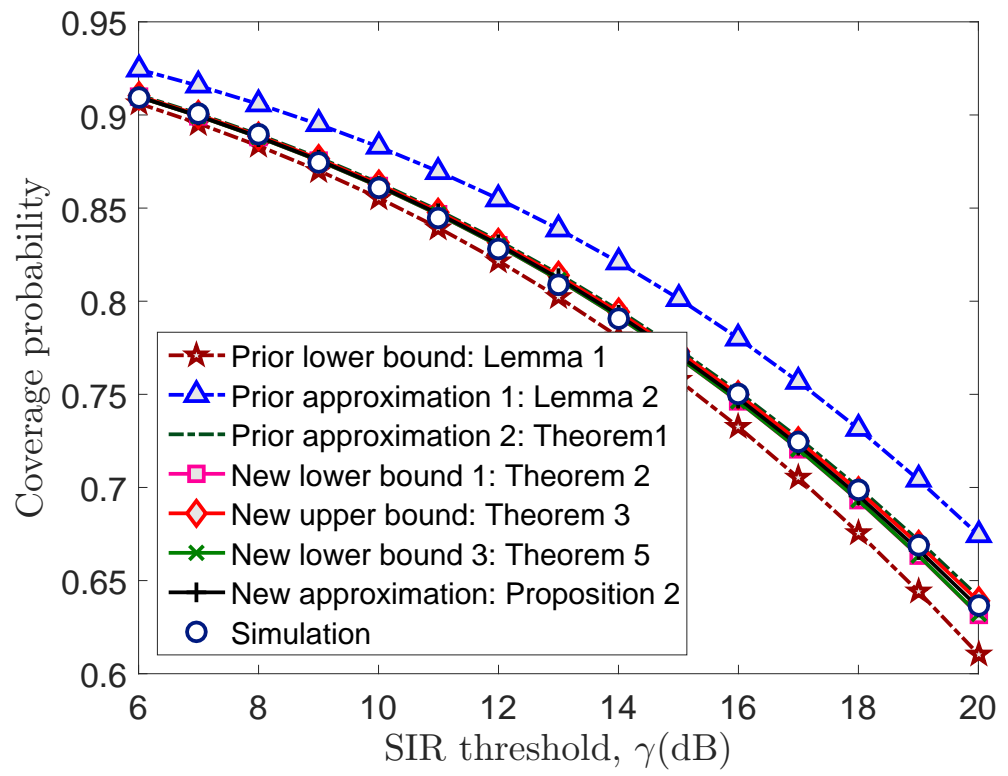

Figure 3.14: Analytical and simulation results for the coverage probability in HD-SH case $\left(\lambda_{1}=0.2\right.$ and $\left.D=0.6\right)$.

of $P$. In Fig. 3.11, we plot the coverage probability of a typical receiver as a function of hole density $\lambda_{1}$ assuming all other parameters are fixed (we assume $D=1$ ). Small values of $\lambda_{1}$ result in LD-SH configuration, whereas high values 


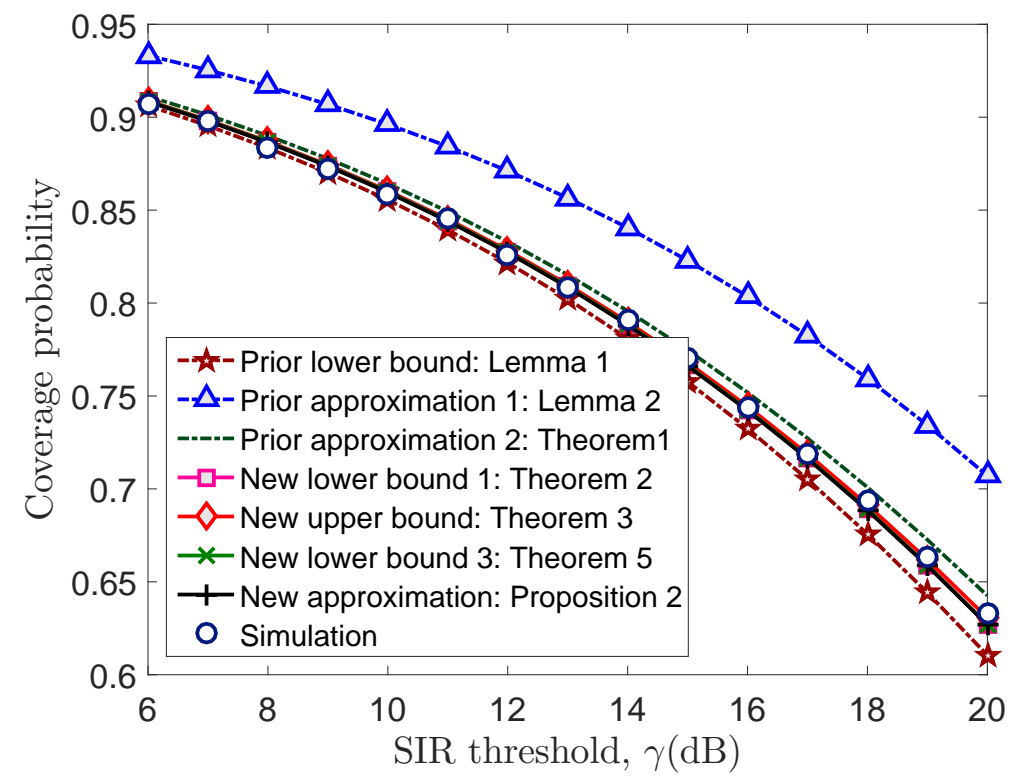

Figure 3.15: Analytical and simulation results for the coverage probability in LD-LH case $\left(\lambda_{1}=0.05\right.$ and $\left.D=1.5\right)$.

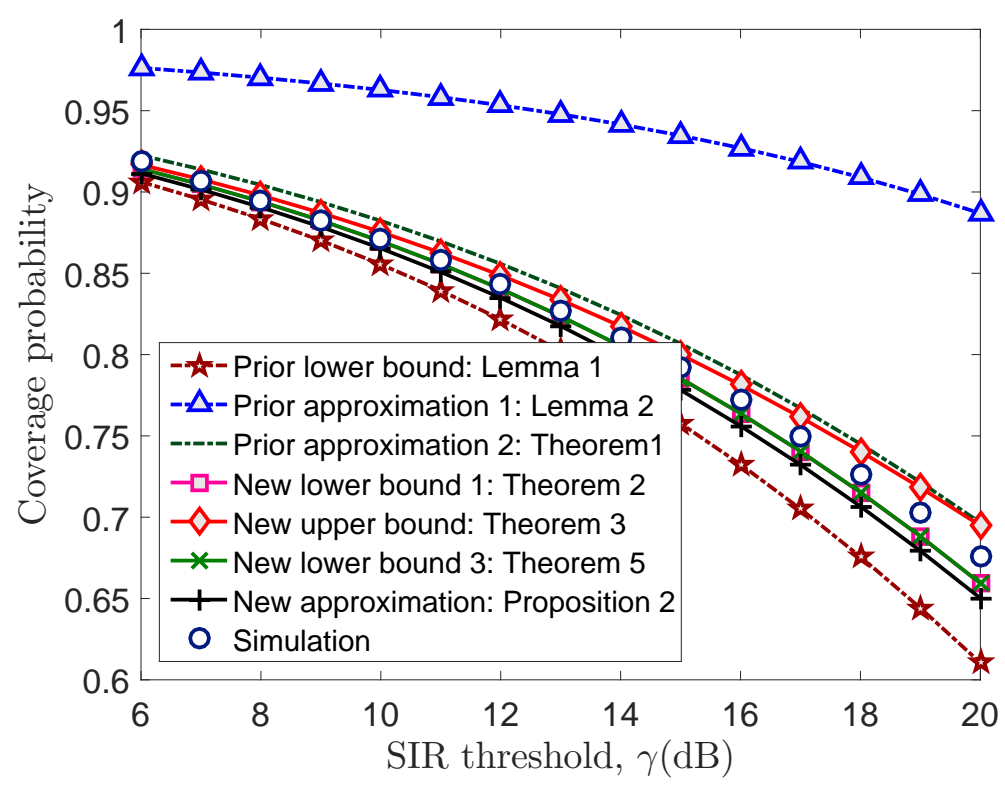

Figure 3.16: Analytical and simulation results for the coverage probability in HD-LH case $\left(\lambda_{1}=0.2\right.$ and $\left.D=1.5\right)$.

result in the HD-SH configuration. In Fig. 3.12, we conduct the same study but instead of varying $\lambda_{1}$, we now vary the hole radius $D$ while fixing $\lambda_{1}=0.1$. The low values of $D$ result in HD-SH configuration, whereas the high values 


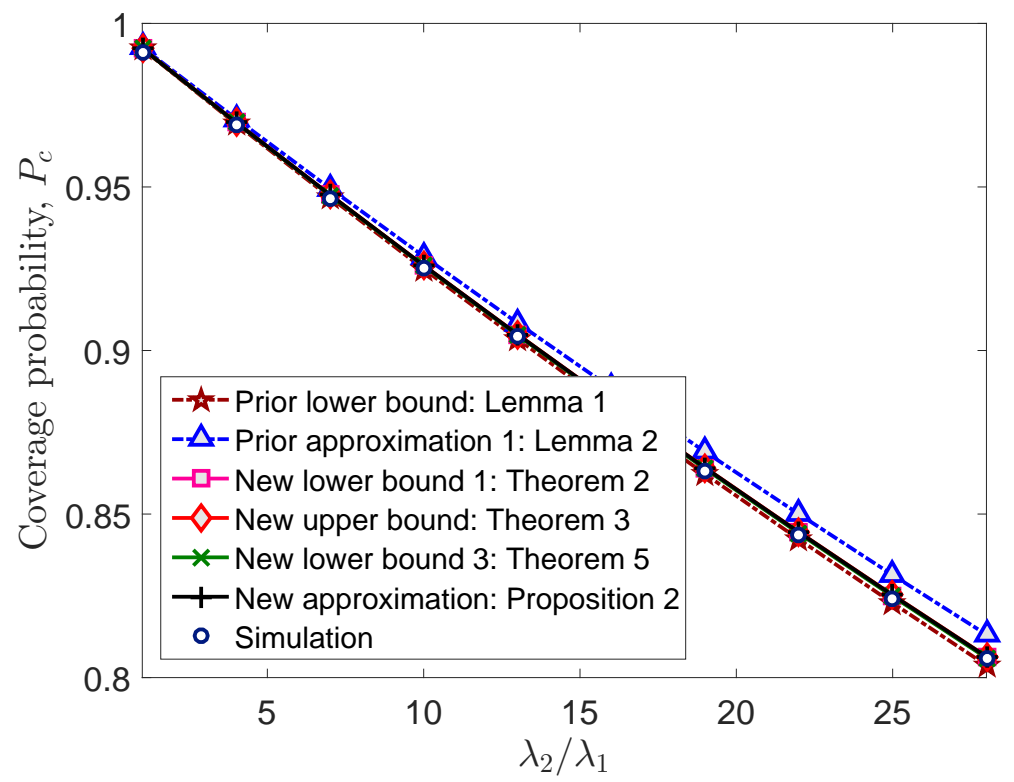

Figure 3.17: Analytical and simulation results for the coverage probabilities of the PHP nodes as a function of $\lambda_{2} / \lambda_{1}$ under configuration LD-SH $\left(\lambda_{1}=0.05\right.$ and $D=0.6)$.

result in HD-LH configuration. Comparison of the proposed results with the simulations reveals that all the bounds and approximations proposed in this chapter are surprisingly tight, even for the extreme configuration like HDLH, where the overlaps are significant. The lower bounds work even better. We also notice that the prior results (given by Lemmas 1 and 2) deviate significantly when the overlaps in the holes are significant. In particular, the approximation given by Lemma 2 becomes very loose. As discussed in Remark 1, this is because its derivation involved independent thinning of the interference field, which distorts the local neighborhood of the typical receiver.

We now plot the analytical and simulation results for the coverage probability of the typical receiver as a function of the SIR threshold in the four possible configurations in Figs. 3.13-3.16. Fig. 3.13 shows the results for 


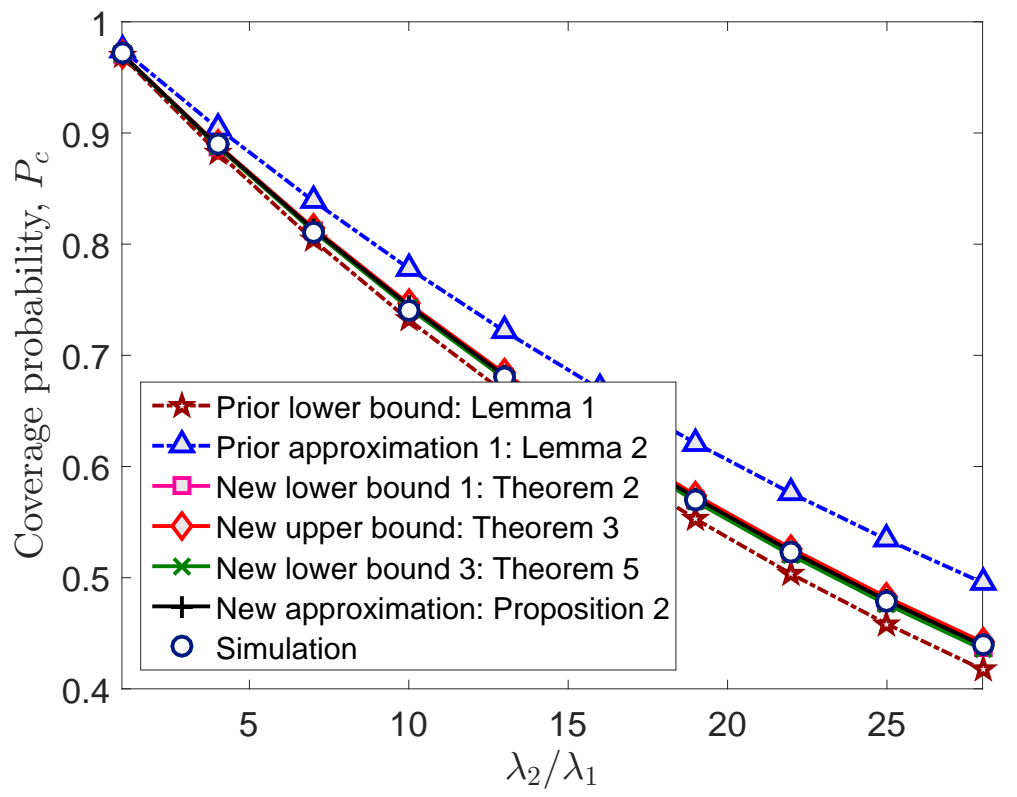

Figure 3.18: Analytical and simulation results for the coverage probabilities of the PHP nodes as a function of $\lambda_{2} / \lambda_{1}$ under configuration HD-SH $\left(\lambda_{1}=0.2\right.$ and $D=0.6)$.

LD-SH case with small holes of radius $D=0.6$ and low density of $\lambda_{1}=0.05$, while results for the HD-SH case are shown in Fig. 3.14 with parameters $D=0.6$ and $\lambda_{1}=0.2$. Further, Fig. 3.15 shows results for the LD-LH case with large holes of radius $D=1.5$ and low density of $\lambda_{1}=0.05$, while the results for the HD-LH case with parameters $D=1.5$ and $\lambda_{1}=0.2$ are shown in Fig. 3.16. The plots again confirm the accuracy of our results and show that the first-order statistic approximation given by Lemma 2 is rather loose while our proposed bounds lead to tight upper and lower bounds in all cases. Interestingly, all the proposed results work so well that they are nearly indistinguishable in all configurations except the most extreme one of HD-LH (Fig. 3.16). In this configuration, we first notice that both the lower bounds given by Theorems 2 and 5 work equally well, which means considering even a 


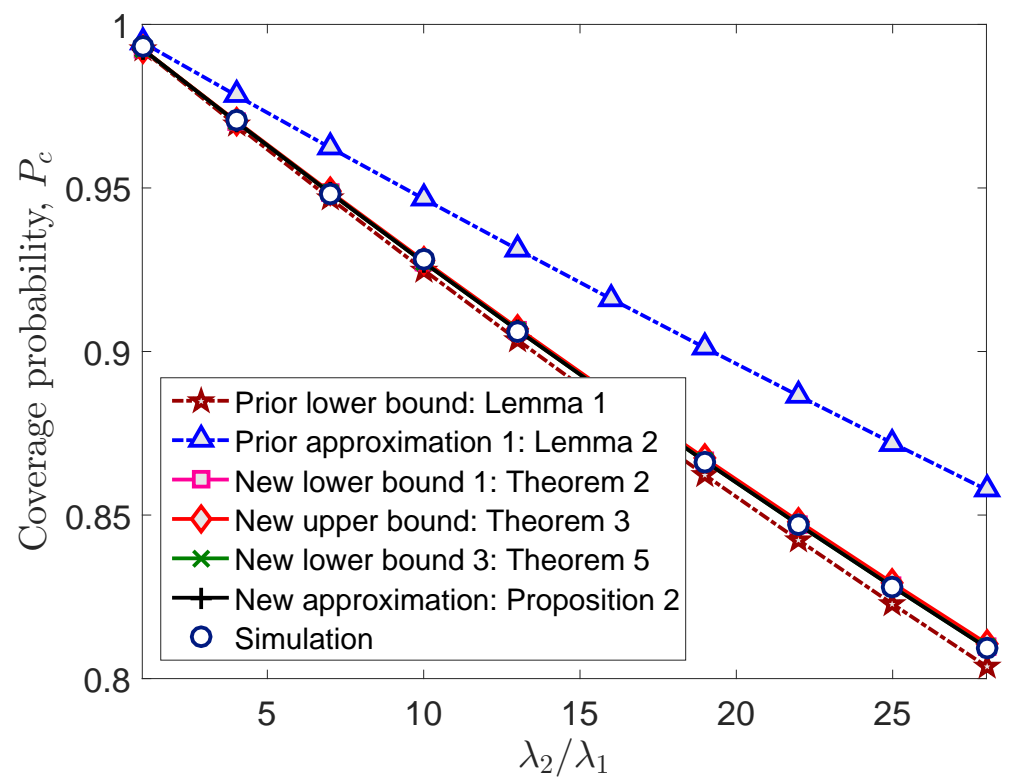

Figure 3.19: Analytical and simulation results for the coverage probabilities of the PHP nodes as a function of $\lambda_{2} / \lambda_{1}$ under configuration LD-LH $\left(\lambda_{1}=0.05\right.$ and $D=1.5)$.

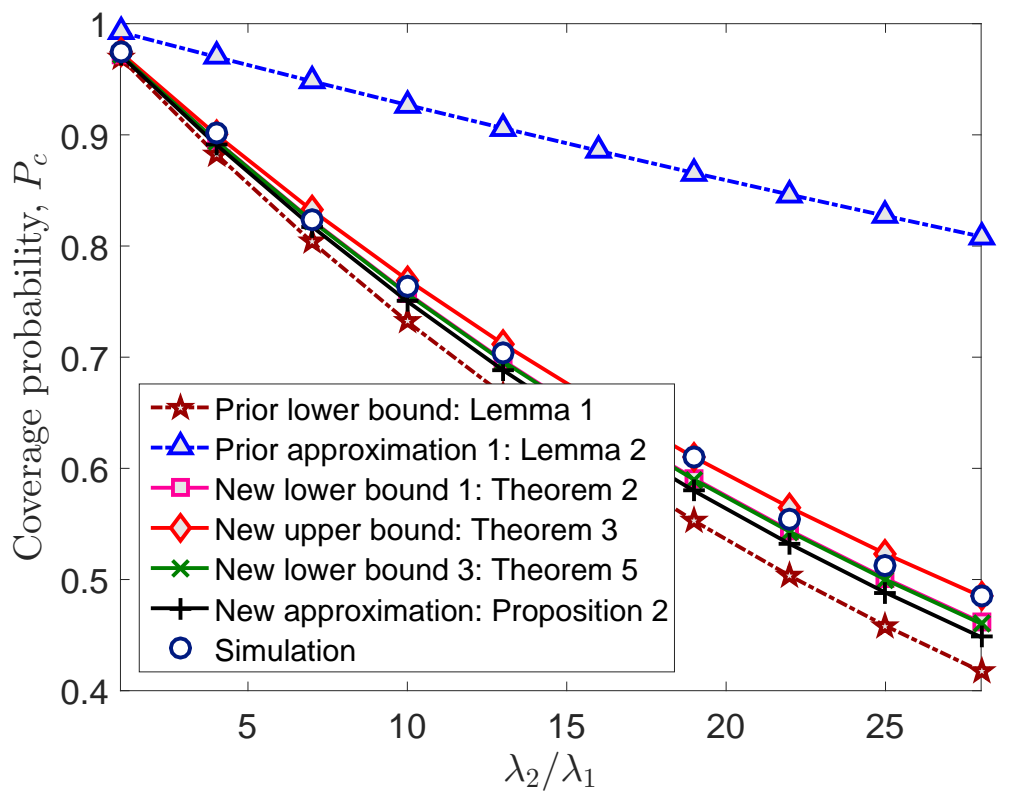

Figure 3.20: Analytical and simulation results for the coverage probabilities of the PHP nodes as a function of $\lambda_{2} / \lambda_{1}$ under configuration HD-LH $\left(\lambda_{1}=0.2\right.$ and $D=1.5)$. 
Chapter 3: Poisson Hole Process: Theory and Applications to WIRELESS NETWORKS

single hole in the interference field accurately is good enough for the accurate characterization of interference. As expected, we also notice that the approximation derived by handling the overlaps in the average sense in Proposition 1 does not work better than the proposed bounds in the extreme configuration of HD-LH (Fig. 3.16). This is because any average-based arguments do not necessarily capture the local neighborhood of the typical node as well as the bounds do.

For completeness, we also plot the analytical and simulation results for the coverage probability of the typical receiver in the PHP as a function of $\lambda_{2} / \lambda_{1}$ in the four configurations in Figs. 3.17-3.20. In Figs. 3.17 and 3.18, design parameters are set in order to simulate LD-SH and HD-SH cases, respectively. In particular, we assume $D=0.6$ and $\lambda_{1}=\{0.05,0.2\}$. Figs. 3.19 and 3.20 depict results for the LD-LH and HD-LH cases. Here we consider $D=1.5$ and $\lambda_{1}=\{0.05,0.2\}$. As was the case in the above results, our proposed lower and upper bounds provide a remarkably accurate characterization of coverage probability. This is because the local neighborhood of the typical node is carefully preserved while deriving these bounds. On the other hand, the prior results, in particular the first-order statistic approximation, leads to a fairly loose result. 


\section{Chapter 4}

\section{Serving Distance and Coverage}

\section{in a PHP-Based Heterogeneous}

\section{Cellular Network}

\subsection{Introduction}

Stochastic geometry has emerged as a powerful tool for the modeling and analysis of HCNs $[73,74]$. The most popular approach is to model the BS locations of an HCN as a superposition of independent PPPs. While this lends tractability to the analysis of key performance metrics, the independent PPP-based model does not capture inherent spatial separation that exists amongst the locations of the BSs belonging to different tiers. In order to 
capture this inter-tier separation in a two-tier $\mathrm{HCN}$, [46, 47] have proposed a new model in which the macro BS locations are modeled by a PPP and the small cell BS locations are modeled around them using a PHP. The PHP model essentially places an exclusion zone of a given radius around each macro BS where small cells cannot lie.

The presence of holes in the interference field makes it difficult to analyze PHP-based models. This has led to several approximations, including approximating a PHP by its baseline PPP, approximating it by a PPP with the same density as the PHP, and approximating it by a Poisson Cluster Process $[30,46-48,75]$. In context of the HCN analysis, this means that the resulting approximate expressions for key metrics, such as coverage probability, are accurate only for a limited range of system parameters. Furthermore, the serving distance distribution in the presence of holes is not easy to characterize. As a result, prior works on the analysis of PHP-based HCNs either assumed the serving distances to be fixed or used curve-fitting to fit their distributions to a Weibull distribution [47].

In chapter 3, we developed new tractable tools for the shot-noise analysis of an interference field modeled as a PHP by carefully preserving the localneighborhood around the receiver location in order to maintain accuracy while approximating the far-field to lend tractability [61]. Using the same general methodology, we develop new tools for the analysis of PHP-based HCNs in this chapter. 


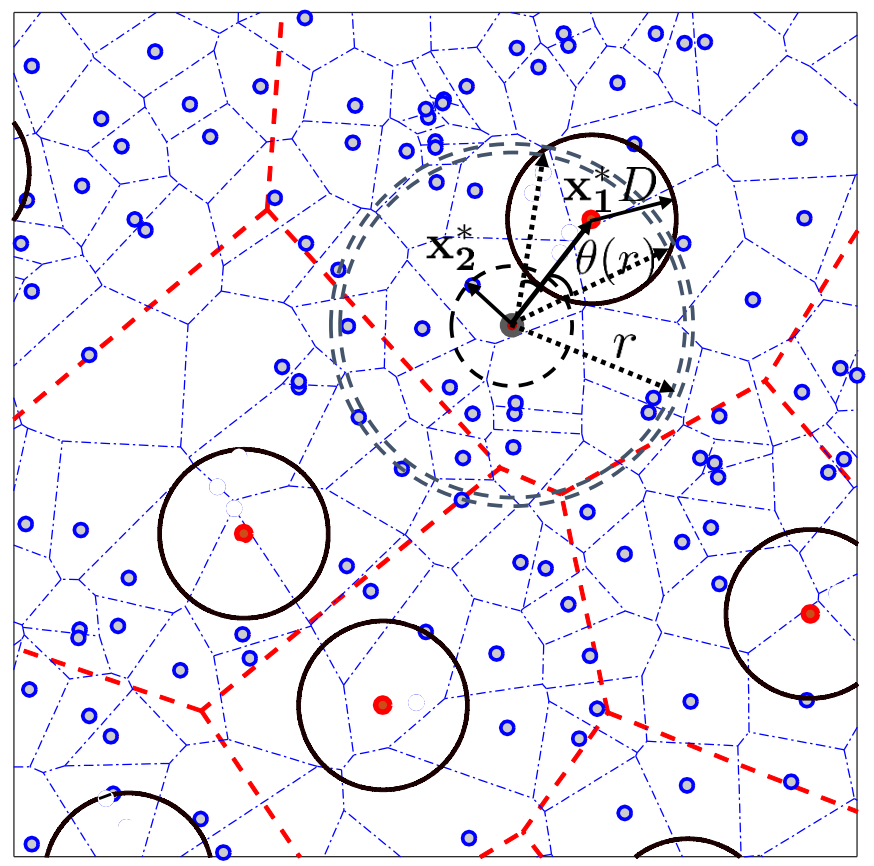

Figure 4.1: Illustration of the PHP-based two-tier HCN system model.

\subsection{System model}

We consider a two-tier HCN consisting of macrocells and small cells. The macro BS locations are modeled as a homogenous $\operatorname{PPP}\left\{\mathbf{x}_{1}\right\} \equiv \Phi_{1}$ with density $\lambda_{1}$ BSs per meter ${ }^{2}$. As proposed in $[46,47]$, we capture the spatial separation among the small cell and macro BSs by modeling the small cell locations as a PHP, which can be defined formally as:

$$
\Phi_{2}=\left\{\mathbf{x}_{2} \in \Omega: \mathbf{x}_{2} \notin \Xi_{D}\right\}=\Omega \backslash \Xi_{D},
$$

where $\Omega$ is a baseline PPP of density $\lambda_{2}$ and $\Xi_{D} \triangleq \bigcup_{\mathbf{x}_{1} \in \Phi_{1}} \mathbf{b}\left(\mathbf{x}_{1}, D\right)$ with $\mathbf{b}\left(\mathbf{x}_{1}, D\right)$ being a ball of radius $D$ centered at $\mathbf{x}_{1}$. In other words, $\Phi_{2}$ is generated by carving out holes of fixed radius $D$ centered around the points 
of $\Phi_{1}$ from $\Omega$. This means the minimum separation between a macro and small cell is $D$. This setup is illustrated in Fig. 4.1.

For simplicity of exposition, we consider macro and small cell users separately. We assume that each macro user is served by its closest macrocell and each small cell user is served by its closest small cell. While this case may appear to be restrictive, its analysis entails significant complexity due to the presence of holes in $\Phi_{2}$. The tools developed in this chapter to handle this complexity also provide first few concrete steps towards the analysis of open access case, which is significantly more complex due to the consideration of cell selection jointly across the two tiers.

The user locations are modeled by an independent stationary point process. The downlink analysis is performed at a typical user located at the origin under two cases: (i) it is served by its closest small cell, and (ii) it is served by its closest macrocell. Denoting the distance from the typical user to its closest BS from the $k^{\text {th }}$ tier by $\left\|\mathbf{x}_{k}^{*}\right\|=Z_{k}$ and transmit power of the $k^{\text {th }}$ tier BSs by $P_{k}$, the received power at the typical user is: $P_{r, k}=P_{k} h_{\mathbf{x}_{k}^{*}} Z_{k}^{-\alpha}$, where $h_{\mathbf{x}_{k}^{*}} \sim \exp (1)$ models Rayleigh fading and $\alpha>2$ is path-loss exponent. The signal to interference ratio (SIR) at the typical user served by a BS of the $k^{\text {th }}$ tier is:

$$
\operatorname{SIR}\left(\left\|\mathbf{x}_{k}^{*}\right\|\right)=\frac{P_{k} h_{\mathbf{x}_{k}^{*}}\left\|\mathbf{x}_{k}^{*}\right\|^{-\alpha}}{\sum_{j=1}^{2} \sum_{\mathbf{x}_{j} \in \Phi_{j} \backslash\left\{\mathbf{x}_{k}^{*}\right\}} P_{j} h_{\mathbf{x}}\left\|\mathbf{x}_{j}\right\|^{-\alpha}},
$$


where $k \in\{1,2\}$. The thermal noise is assumed to be negligible compared to the interference and is hence ignored.

\subsection{Coverage Probability}

This is the main technical section of this chapter where we first derive the distribution of the distance from the typical user to its closest macro and small cell BSs (denoted by $Z_{1}$ and $Z_{2}$ ). Using these distributions, we will derive expressions for the coverage probability of the typical user in the two cases.

\subsubsection{Distributions of serving distances $Z_{1}$ and $Z_{2}$}

Recall that $Z_{1}$ denotes the distance of the typical user (placed at the origin) to its closest macrocell (closest point of $\Phi_{1}$ ). The distribution of $Z_{1}$ is wellknown and can be derived using the null probability of a PPP [78]. For this case, it comes out to be

$$
f_{Z_{1}}\left(z_{1}\right)=2 \pi \lambda_{1} z_{1} \exp \left(-\pi \lambda_{1} z_{1}^{2}\right), \quad z_{1}>0
$$

Also recall that $Z_{2}$ denotes the distance of the typical user to its closest small cell. Its distribution is much more complex to characterize. In order to curtail the complexity, we consider only the closest hole to the typical user in this 
Chapter 4: Serving Distance and Coverage in a PHP-Based Heterogeneous Cellular Network

derivation and ignore all other holes. In other words, for the derivation of this distance distribution, PHP $\Phi_{2}$ is approximated by $\Omega$ with only one (closest) hole carved out. This approach clearly underestimates $Z_{2}$ and the resulting random variable is denoted by $\hat{Z}_{2}$.

The analytical result corresponding to distribution of distance $\hat{Z}_{2}$ is given by Lemma 7 which its accuracy is investigated in Fig. 4.2. The proof is provided in Appendix B.1.

Lemma 7 The probability density function (PDF) of distance $\hat{Z}_{2}$ is given by

$$
\begin{aligned}
& f_{\hat{Z_{2}} \mid Z_{1} \leq D}\left(\hat{z_{2}}\right)= \\
& \left\{\begin{array}{cc}
\left(2 \pi \lambda_{2} \hat{z_{2}}-\lambda_{2} \frac{\mathrm{d} A_{\text {ins }}\left(\hat{z_{2}}, Z_{1}\right)}{\mathrm{d} \hat{z_{2}}}\right) \exp \left(-\lambda_{2}\left(\pi{\hat{z_{2}}}^{2}-A_{\text {ins }}\left(\hat{z_{2}}, Z_{1}\right)\right)\right) & D-Z_{1}<\hat{z_{2}} \leq Z_{1}+D \\
2 \pi \lambda_{2} \hat{z_{2}} \exp \left(-\pi \lambda_{2}\left({\hat{z_{2}}}^{2}-D^{2}\right)\right) & \hat{z_{2}}>Z_{1}+D
\end{array}\right.
\end{aligned}
$$

and

$$
\begin{aligned}
& f_{\hat{Z_{2}} \mid Z_{1}>D}\left(\hat{z_{2}}\right)= \\
& \left\{\begin{array}{cc}
2 \pi \lambda_{2} \hat{z_{2}} \exp \left(-\pi \lambda_{2}{\hat{z_{2}}}^{2}\right) & \hat{z_{2}} \leq Z_{1}-D \\
\left(2 \pi \lambda_{2} \hat{z_{2}}-\lambda_{2} \frac{\mathrm{d} A_{\text {ins }}\left(\hat{z_{2}}, Z_{1}\right)}{\mathrm{d} \hat{z_{2}}}\right) \exp \left(-\lambda_{2}\left(\pi{\hat{z_{2}}}^{2}-A_{\text {ins }}\left(\hat{z_{2}}, Z_{1}\right)\right)\right) & Z_{1}-D<\hat{z_{2}} \leq Z_{1}+D \\
2 \pi \lambda_{2} \hat{z_{2}} \exp \left(-\pi \lambda_{2}\left({\hat{z_{2}}}^{2}-D^{2}\right)\right) & \hat{z_{2}}>Z_{1}+D
\end{array}\right.
\end{aligned}
$$

where $\hat{z_{2}}$ and $Z_{1}$ denote the distance from the typical user to its serving 
small cell BS and its distance to the closest macro BS, respectively. Further $A_{\text {ins }}\left(\hat{z_{2}}, Z_{1}\right)=\mathcal{B}\left(D, \hat{z_{2}}, Z_{1}\right)+\mathcal{B}\left(\hat{z_{2}}, D, Z_{1}\right)-0.5 \mathcal{A}\left(D, \hat{z_{2}}, Z_{1}\right)$ denotes the area of intersection between two circles $\mathbf{b}\left(0, \hat{z_{2}}\right)$ and $\mathbf{b}\left(Z_{1}, D\right)$. Other functions appearing in the above expression are as follows:

$$
\begin{aligned}
\mathcal{A}(\kappa, \zeta, \eta) & =\sqrt{\left(\kappa^{2}-(\eta-\zeta)^{2}\right)\left((\zeta+\eta)^{2}-\kappa^{2}\right)}, \mathcal{B}(\kappa, \zeta, \eta)=\kappa^{2} \arccos \left(\frac{\kappa^{2}-\zeta^{2}+\eta^{2}}{2 \kappa \eta}\right) \\
\text { and } \mathcal{C}(\kappa, \zeta, \eta) & =\frac{\kappa^{2}-\zeta^{2}+\eta^{2}}{2 \kappa^{2} \eta} .
\end{aligned}
$$

Using these results, we now focus on the derivation of coverage probability in the two cases:

(i) typical user served by the macro tier $\left(\mathrm{P}_{\mathrm{c} 1}\right)$, and (ii) typical user served by the small cell tier $\left(\mathrm{P}_{\mathrm{c} 2}\right)$.

Coverage probability $\mathrm{P}_{c k}$ is defined as the probability that the instantaneous SIR of a typical user when it is served by the $k^{\text {th }}$ tier BS (denoted by $\left.\operatorname{SIR}\left(Z_{k}\right)\right)$ is greater than a target $\operatorname{SIR} \gamma$. It is mathematically defined as

$$
\mathrm{P}_{\mathrm{ck}}=\mathbb{E}_{Z_{k}}\left[\mathbb{P}\left\{\operatorname{SIR}\left(z_{k}\right) \geq \gamma \mid Z_{k}\right\}\right]=\int_{z_{k}>0} \mathbb{P}\left\{\operatorname{SIR}\left(z_{k}\right) \geq \gamma\right\} f_{Z_{k}}\left(z_{k}\right) \mathrm{d} z_{k}
$$

Key challenge in the derivation of $\mathrm{P}_{\mathrm{c} k}$ is the presence of holes (exclusion zones) in the PHP $\Phi_{2}$. Recently, we developed new tools to handle these exclusion zones in an ad hoc network modeled by a PHP in [61]. We extend these tools to derive remarkably right bounds and approximations for the coverage probability $\mathrm{P}_{c k}$. More details are provided next. 
Chapter 4: Serving Distance and Coverage in a PHP-Based Heterogeneous Cellular Network

\subsubsection{Coverage probability when the typical user is served by its closest macro cell}

In this case, the set of BSs contributing to interference are macro BSs from $\Phi_{1} \backslash \mathbf{x}_{1}^{*}$ and small BSs from $\Phi_{2}$. We first derive the coverage probability of the typical user by considering only the closest hole to the typical user. Thereby, the interference field of small cell BSs is overestimated by $\Omega \cap \mathbf{b}^{c}\left(\mathbf{x}_{1}^{*}, D\right)$. This provides a lower bound on the coverage probability, which is derived next.

Theorem 6 Considering only the closest hole in the interference field of small cells, the coverage probability of a typical user when it connects to its closest macro $B S$ is

$$
\begin{aligned}
\mathrm{P}_{\mathrm{c} 1} & \geq \int_{0}^{D} \mathcal{G}_{1}^{(1)}(s) \mathcal{G}_{2}^{(1)}\left(s, z_{1}\right) \mathcal{G}_{3}^{(1)}\left(s, z_{1}\right) f_{Z_{1}}\left(z_{1}\right) \mathrm{d} z_{1} \\
& +\int_{D}^{\infty} \hat{\mathcal{G}}_{1}^{(1)}(s) \mathcal{G}_{2}^{(1)}\left(s, z_{1}\right) \mathcal{G}_{3}^{(1)}\left(s, z_{1}\right) f_{Z_{1}}\left(z_{1}\right) \mathrm{d} z_{1},
\end{aligned}
$$

$$
\begin{aligned}
& \text { where } s=\frac{\gamma z_{1}^{\alpha}}{P_{1}}, \mathcal{G}_{1}^{(1)}(s)=\exp \left(\int_{D-z_{1}}^{\infty} \frac{-2 \pi \lambda_{2} r \mathrm{~d} r}{1+\frac{r^{\alpha}}{s P_{2}}}\right), \hat{\mathcal{G}}_{1}^{(1)}(s)=\exp \left(-\pi \lambda_{2} \frac{\left(s P_{2}\right)^{2 / \alpha}}{\operatorname{sinc}(2 / \alpha)}\right) \\
& \mathcal{G}_{2}^{(1)}\left(s, z_{1}\right)=\exp \left(\int_{\left|z_{1}-D\right|}^{z_{1}+D} \arccos \left(\frac{r^{2}+z_{1}^{2}-D^{2}}{2 z_{1} r}\right) \frac{2 \lambda_{2}}{1+\frac{r^{\alpha}}{P_{2} s}} r \mathrm{~d} r\right), \mathcal{G}_{3}^{(1)}\left(s, z_{1}\right)=\exp (- \\
& \left.2 \pi \lambda_{1} \int_{z_{1}}^{\infty}\left(1-\frac{1}{1+s P_{1} v^{-\alpha}}\right) v \mathrm{~d} v\right) .
\end{aligned}
$$

Proof: See Appendix B.2.

The tightness of this bound will be demonstrated in the numerical results section (see Fig. 4.3).

We now extend the above approach to incorporate all the holes in the 
interference field. In order to maintain tractability, we ignore the effect of overlaps among the holes such that each hole is assumed to be carved out individually from the baseline PPP instead of carving out the union of the holes. Some of the points of the baseline PPP located inside the holes may be virtually removed multiple times by using this approach, thus leading to underestimation of the interference power experienced by the typical user from small cell BSs. This leads to an upper bound on the coverage probability. Please refer to [61] for more details where we proposed this bounding technique for the analysis of ad hoc networks.

Theorem 7 The coverage probability of a typical user which is served by its closest macro BS is upper bounded by

$$
\begin{aligned}
\mathrm{P}_{\mathrm{c} 1} & \leq \int_{0}^{D} \mathcal{G}_{1}^{(1)}(s) \mathcal{G}_{2}^{(1)}\left(s, z_{1}\right) \mathcal{G}_{4}^{(1)}\left(s, z_{1}\right) f_{Z_{1}}\left(z_{1}\right) \mathrm{d} z_{1} \\
& +\int_{D}^{\infty} \hat{\mathcal{G}}_{1}^{(1)}(s) \mathcal{G}_{2}^{(1)}\left(s, z_{1}\right) \mathcal{G}_{4}^{(1)}\left(s, z_{1}\right) f_{Z_{1}}\left(z_{1}\right) \mathrm{d} z_{1}
\end{aligned}
$$

$$
\begin{aligned}
& \text { where } s=\frac{\gamma z_{1}^{\alpha}}{P_{1}}, \mathcal{G}_{1}^{(1)}(s)=\exp \left(\int_{D-z_{1}}^{\infty} \frac{-2 \pi \lambda_{2} r \mathrm{~d} r}{1+\frac{r \alpha}{s P_{2}}}\right), \hat{\mathcal{G}}_{1}^{(1)}(s)=\exp \left(-\pi \lambda_{2} \frac{\left(s P_{2}\right)^{2 / \alpha}}{\operatorname{sinc}(2 / \alpha)}\right) \\
& \mathcal{G}_{2}^{(1)}\left(s, z_{1}\right)=\exp \left(f\left(s, z_{1}\right)\right), f\left(s, z_{1}\right)=\int_{\left|z_{1}-D\right|}^{z_{1}+D} \arccos \left(\frac{r^{2}+z_{1}^{2}-D^{2}}{2 z_{1} r}\right) \frac{2 \lambda_{2}}{1+\frac{r^{\alpha}}{P_{2} s}} r \mathrm{~d} r, \zeta(s, v) \\
& =\frac{1}{1+s P_{1} v^{-\alpha}}, \mathcal{G}_{4}^{(1)}\left(s, z_{1}\right)=\exp \left(-2 \pi \lambda_{1} \int_{z_{1}}^{\infty}(1-\exp (f(s, v)) \zeta(s, v)) v \mathrm{~d} v\right) .
\end{aligned}
$$

Proof: See Appendix B.3. 
Chapter 4: Serving Distance and Coverage in a PHP-Based

\subsubsection{Coverage probability when the typical user is served}

\section{by its closest small cell}

In this case, the set of interfering BSs constitutes macro BSs from $\Phi_{1}$ and small cell BSs from $\Phi_{2} \backslash \mathbf{x}_{2}^{*}$ where $\mathbf{x}_{2}^{*}$ denotes the serving small cell BS. As was the case in Theorem 6, we again consider only the effect of the closest hole to the typical user, thus overestimating the interference field. For the distribution of serving distance $Z_{2}$, we use its approximation derived in Lemma 7 . The resulting approximation for the coverage probability is provided next.

Theorem 8 Considering only the closest hole in the interference field, the coverage probability of the typical user which connects to its closest small cell $B S$ is approximated by

$\mathrm{P}_{\mathrm{c} 2} \simeq$

$\int_{0}^{D} \int_{D-z_{1}}^{\infty} \mathcal{G}_{1}^{(2)}\left(s, \hat{z_{2}}\right) \mathcal{G}_{2}^{(2)}\left(s, z_{1}, \hat{z_{2}}\right) \mathcal{G}_{3}^{(2)}\left(s, z_{1}\right) \mathcal{G}_{4}^{(2)}\left(s, z_{1}\right) f_{\hat{Z}_{2} \mid Z_{1} \leq D}\left(\hat{z_{2}}\right) f_{Z_{1}}\left(z_{1}\right) \mathrm{d} \hat{z_{2}} \mathrm{~d} z_{1}+$ $\int_{D}^{\infty} \int_{0}^{\infty} \mathcal{G}_{1}^{(2)}\left(s, \hat{z_{2}}\right) \mathcal{G}_{2}^{(2)}\left(s, z_{1}, \hat{z_{2}}\right) \mathcal{G}_{3}^{(2)}\left(s, z_{1}\right) \mathcal{G}_{4}^{(2)}\left(s, z_{1}\right) f_{\hat{Z}_{2} \mid Z_{1}>D}\left(\hat{z_{2}}\right) f_{Z_{1}}\left(z_{1}\right) \mathrm{d} \hat{z_{2}} \mathrm{~d} z_{1}$

$$
\begin{aligned}
& \text { where } s=\frac{\gamma \hat{z_{2}} \alpha}{P_{2}}, \mathcal{G}_{1}^{(2)}\left(s, \hat{z_{2}}\right)=\exp \left(\int_{\hat{z_{2}}}^{\infty} \frac{-2 \pi \lambda_{2} r \mathrm{dr}}{1+\frac{r}{s P_{2}}}\right), \mathcal{G}_{2}^{(2)}\left(s, z_{1}, \hat{z_{2}}\right)=\exp \left(g\left(s, z_{1}, \hat{z_{2}}\right)\right) \\
& \text { which in } g\left(s, z_{1}, \hat{z_{2}}\right)=\exp \left(\int_{\max \left(\hat{z_{2}},\left|z_{1}-D\right|\right)}^{\max \left(z_{1}+D\right)} \operatorname{arcos}\left(\frac{r^{2}+z_{1}^{2}-D^{2}}{2 z_{1} r}\right) \frac{2 \lambda_{2}}{1+\frac{r^{\alpha}}{s P_{2}}} r \mathrm{~d} r\right), \mathcal{G}_{3}^{(2)}\left(s, z_{1}\right) \\
& =\zeta\left(s, z_{1}\right)=\frac{1}{1+s P_{1} z_{1}^{-\alpha}}, \mathcal{G}_{4}^{(2)}\left(s, z_{1}\right)=\exp \left(-2 \pi \lambda_{1} \int_{z_{1}}^{\infty}(1-\zeta(s, v)) v \mathrm{~d} v\right) .
\end{aligned}
$$

Proof: See Appendix B.4. 
The tightness of this approximation will be demonstrated in the numerical results section (see Fig. 4.3).

On the same lines as Theorem 7 , we now provide another approximation for the coverage probability where we underestimate the interference power from the small cells by carving out holes in a PHP individually without considering overlaps amongst them. The resulting approximation is provided next.

Theorem 9 The coverage probability of a typical user which is served by its closest small cell BS is approximated by

$\mathrm{P}_{\mathrm{c} 2} \simeq$

$$
\begin{gathered}
\int_{0}^{D} \int_{D-z_{1}}^{\infty} \mathcal{G}_{1}^{(2)}\left(s, \hat{z_{2}}\right) \mathcal{G}_{2}^{(2)}\left(s, z_{1}, \hat{z_{2}}\right) \mathcal{G}_{3}^{(2)}\left(s, z_{1}\right) \mathcal{G}_{5}^{(2)}\left(s, z_{1}, \hat{z_{2}}\right) f_{\hat{Z}_{2} \mid Z_{1} \leq D}\left(\hat{z_{2}}\right) f_{Z_{1}}\left(z_{1}\right) \mathrm{d} \hat{z_{2}} \mathrm{~d} z_{1}+ \\
\int_{D}^{\infty} \int_{0}^{\infty} \mathcal{G}_{1}^{(2)}\left(s, \hat{z_{2}}\right) \mathcal{G}_{2}^{(2)}\left(s, z_{1}, \hat{z_{2}}\right) \mathcal{G}_{3}^{(2)}\left(s, z_{1}\right) \mathcal{G}_{5}^{(2)}\left(s, z_{1}, \hat{z_{2}}\right) f_{\hat{Z_{2} \mid Z_{1}>D}}\left(\hat{z_{2}}\right) f_{Z_{1}}\left(z_{1}\right) \mathrm{d} \hat{z_{2}} \mathrm{~d} z_{1}
\end{gathered}
$$

$$
\begin{aligned}
& \text { where } s=\frac{r \hat{z}_{2}^{\alpha}}{P_{2}}, \mathcal{G}_{1}^{(2)}\left(s, \hat{z_{2}}\right)=\exp \left(\int_{\hat{z_{2}}}^{\infty} \frac{-2 \pi \lambda_{2} r \mathrm{dr}}{1+\frac{r \alpha}{s P_{2}}}\right), \mathcal{G}_{2}^{(2)}\left(s, z_{1}, \hat{z_{2}}\right)=\exp \left(g\left(s, z_{1}, \hat{z_{2}}\right)\right) \\
& \text { which in } g\left(s, z_{1}, \hat{z_{2}}\right)=\exp \left(\int_{\max \left(\hat{z_{2}},\left|z_{1}-D\right|\right)}^{\max \left(\hat{z_{1}}\right)} \arccos \left(\frac{r^{2}+z_{1}^{2}-D^{2}}{2 z_{1} r}\right) \frac{2 \lambda_{2}}{1+\frac{r^{\alpha}}{s P_{2}}} r \mathrm{~d} r\right), \mathcal{G}_{3}^{(2)}\left(s, z_{1}\right) \\
& =\zeta\left(s, z_{1}\right)=\frac{1}{1+s P_{1} z_{1}^{-\alpha}}, \mathcal{G}_{5}^{(2)}\left(s, z_{1}\right)=\exp \left(-2 \pi \lambda_{1} \int_{z_{1}}^{\infty}(1-\exp (g(s, v)) \zeta(s, v)) v \mathrm{~d} v\right) .
\end{aligned}
$$


Chapter 4: Serving Distance and Coverage in a PHP-Based

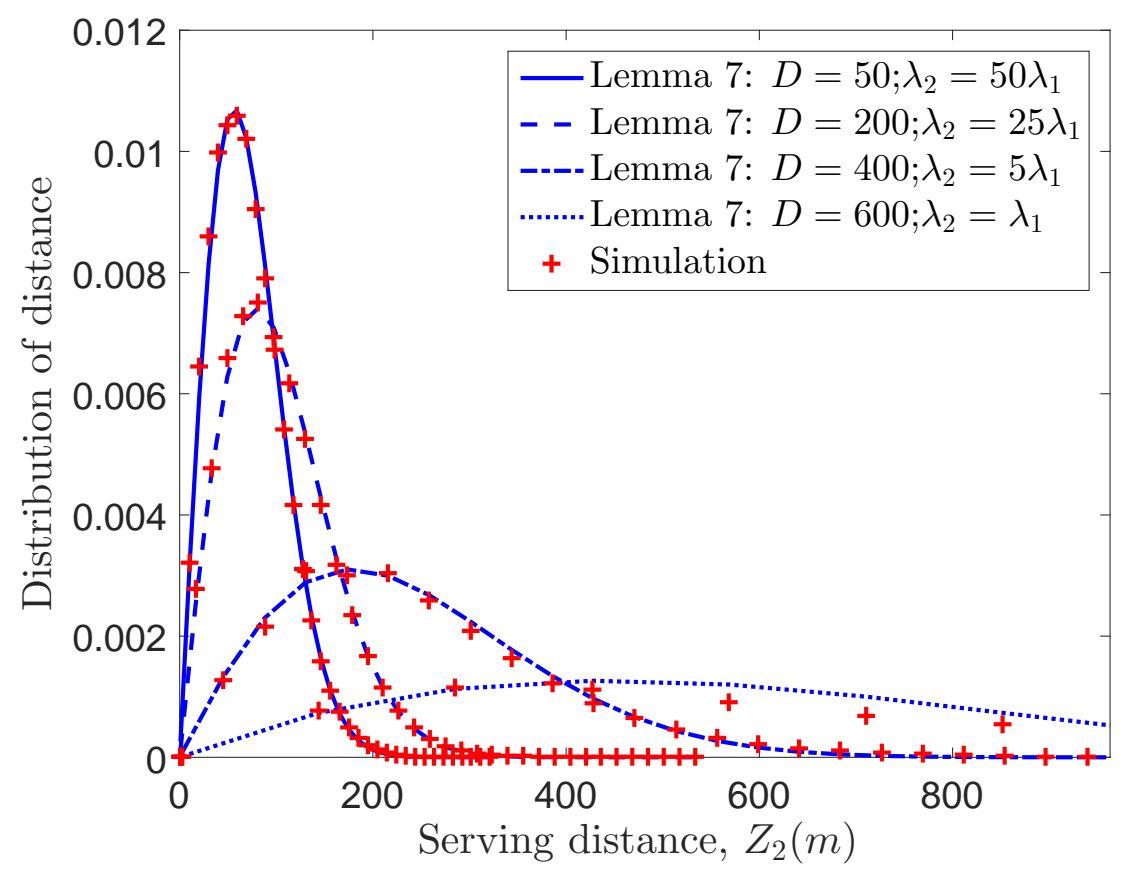

Figure 4.2: Distribution of the distance from the typical user to its closest small cell BS in a PHP.

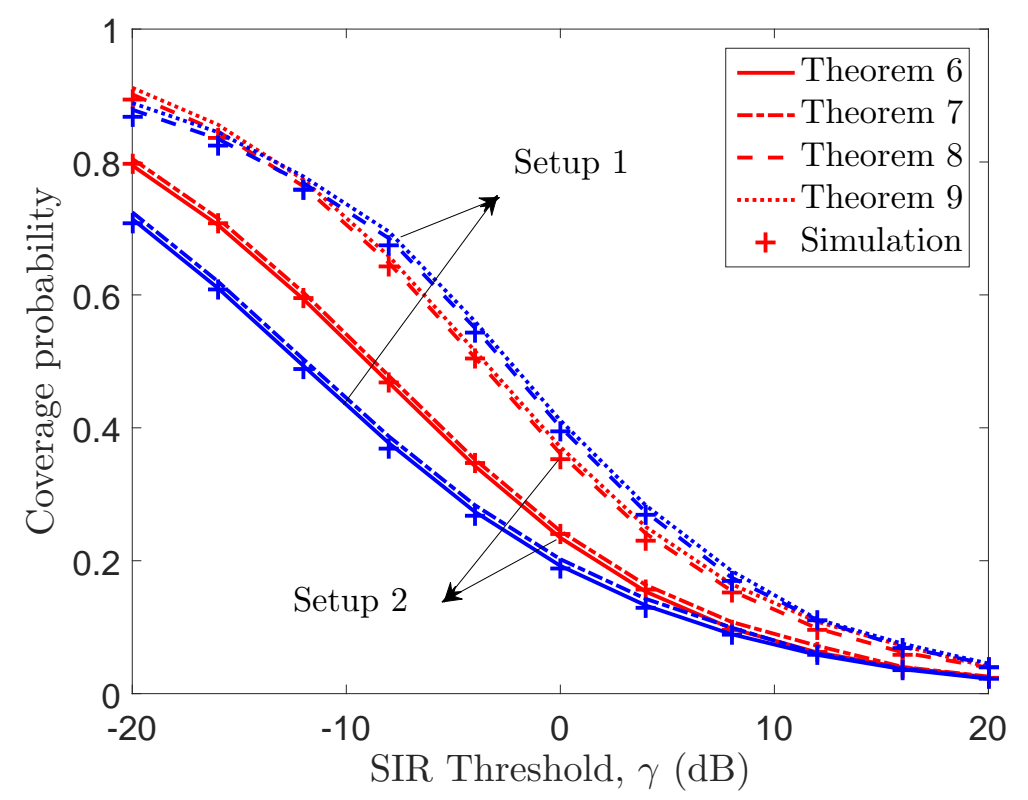

Figure 4.3: Comparison of macro and small cell coverage probability results.

\subsection{Numerical Results and Discussion}

In this section, we validate our results by comparing them against results obtained from Monte Carlo simulations. The parameter settings are based 


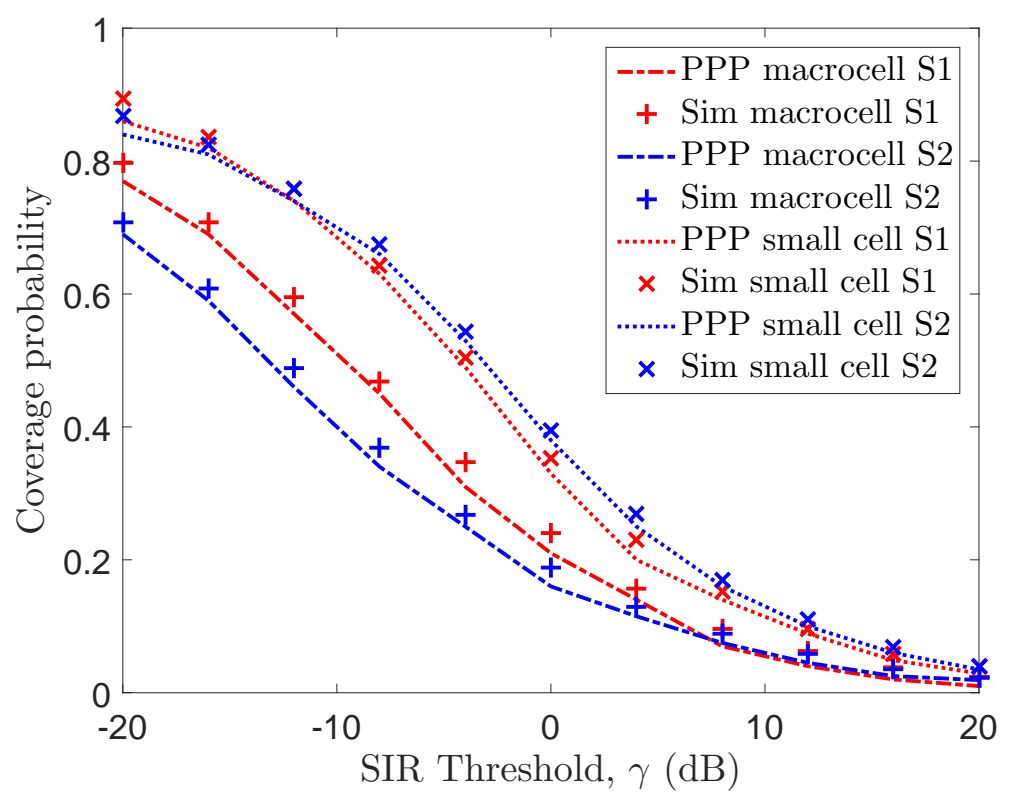

Figure 4.4: Comparison of PPP macro and small cell and PHP-based HCN coverage probability results.

on 3GPP specifications [47] and [79]. We consider two setups such that in both cases, $\lambda_{1}=1 \mathrm{BS} / \mathrm{km}^{2}, \alpha=4$. In setup $1, \lambda_{2}=50 \lambda_{1}, P_{1}=10^{3} P_{2}$ and $D=50 \mathrm{~m}$, while in the setup $2, \lambda_{2}=25 \lambda_{1}, P_{1}=10^{2} P_{2}$ and $D=200 \mathrm{~m}$. As illustrated in Fig. 4.2, the distribution of the distance $\hat{Z}_{2}$ derived in Lemma 7 which underestimated the true serving distance $Z_{2}$ is surprisingly tight for all configurations (even when the density of small cells is low and the radius of exclusion zone $D$ is large). Fig. 4.3 presents the coverage probability of typical user under two cases: (i) served by macrocell, and (ii) served by small cell, as a function of the SIR threshold. Here, we plot the four analytical results given by Theorems $6,7,8$ and 9 , and compare them with results obtained from simulations. We note that all the analytical results are surprisingly tight. As expected, the coverage probability of the users served by the small cells in this 
Chapter 4: Serving Distance and Coverage in a PHP-Based Heterogeneous Cellular Network

setup is higher (due to lower serving distance). Fig 4.4 shows a comparison between the PPP as a popular previous approach and simulation results. This approach leads to a lower bound for the coverage probability of the typical user in a two-tier HCN. 


\section{Chapter 5}

\section{Modeling and Analysis of}

\section{Asymmetric Exclusion Zones}

\subsection{Introduction}

Next generation communication networks are expected to integrate heterogeneous wireless technologies to support massive data requirements and seamless connectivity. The performance of wireless systems can be expressed as a function of SINR which is a useful quantification including the interference term. The accurate characterization of the interference or SINRs in arbitrary distributed node deployments is a challenging problem [70]. Stochastic geometry as a robust tool can develop a mathematical framework for the modeling and analysis of key performance metrics of the wireless network [65]. Furthermore, applying proper interference management techniques is neces- 
Chapter 5: Modeling and Analysis of Asymmetric ExClusion

sary in deploying heterogeneous cellular networks especially for the scenarios in which different systems coexisting in the same spectrum. Applying some of these techniques can establish exclusion zones which protect the communication of the system by inhibiting other concurrent transmissions within the zone. The common approach to evaluate the performance of the system is to assume symmetric interference field which often leads to the analytical tractable model. On the other hand, spatial PPP is a very common and analytically convenient model for the node distributions in large wireless networks. Key system performance metrics like coverage probability and average rate can be derived by averaging over the network realizations. In chapter 3 and 4, we focused on the modeling and analysis of interference management techniques, which introduce some form of spatial interaction among active transmitters. The resulting interference field has been modeled as a PHP.

In many realistic networks, asymmetric interference can be formed by establishing exclusion zones which in the user is distributed arbitrary in the typical cell. This scenario is applicable in various applications like IEEE 802.22 Digital TV [52]- [54] and device-to-device (D2D) communication in cellular networks [50]- [49]. Moreover, using CSMA protocols in these networks leads to the Matérn hard core process which can be evaluated under the same model [56]- [57]. Owing to the more complicated analysis of asymmetric interference field, most of the existing literatures often consider the typical user at the origin [59]. The outage probability of an arbitrary located 
typical user surrounded by a circular exclusion zone conditioned on the locations of the interfering nodes and the fading parameter is derived in [60]. Although unconditional outage probability is obtained by taking the spatial distribution of the nodes, the analytical results are just presented for the special case of the typical user located at the center of the network region. This configuration which includes a circular exclusion zone around the typical transmitter is addressed in [80] by using the well known existing method, i.e., moment generating functions of interference. Although under specific design parameters, a closed form expression is derived, for general realizations the proposed approach leads to intractable analytical results.

The contributions of this section are summarized as follows. First, we propose a novel tractable framework for the modeling and analysis of the asymmetric interference field with our main focus on the exclusion zone for protecting the communication of an arbitrary located user. This approach works based on a transformation from asymmetric interference field in PPP into the symmetric non-homogeneous PPP. Then, as a result of applying this transformation, the Laplace transform of interference field is derived. We provide accurate tractable expressions for the outage probability of the typical user. Then we present closed-form expressions for the analysis by using the second order of Taylor series expansion. We also derive the expressions by the analysis of the scenario in which interfering nodes are distributed at random inside a given finite region. Then, we study the accuracy of the proposed 
approach by means of simulation.

\subsection{Network Model}

\subsubsection{System Model}

We consider a wireless network composed of primary and secondary systems. They operate at the same frequency channel. Secondary transmitters have perfect knowledge of primary network. Hence, the location of primary users and other corresponding network design parameters are available at secondary transmitters. Secondary transmitters are spatially distributed according to the homogeneous PPP $\Phi_{\mathrm{c}}$ of density $\lambda_{\mathrm{c}}$. We assume that each transmitter communicates with a user located at fixed distance $r_{\mathrm{c}}$ from corresponding transmitter in a random orientation. We consider a single primary transmitter located at $\mathbf{x}_{\mathrm{p}}$ communicating with the primary user within a circular region of radius $D$. There is an exclusion zone around the primary cell, within which secondary transmitters are inactive. In this system, the typical user can be located at an arbitrary location inside the exclusion zone. This modeling leads to an asymmetric interference field which is analytically intractable problem. Interference management techniques can define exclusion zone of radius $D$ around the primary cell. In other words, an interfering node outside the exclusion zone can contribute to the aggregate interference power at the typical user. Let $r_{\mathrm{p}}$ be the distance of primary user inside the 
primary cell with radius $D$ from corresponding transmitter located at the center, $\left\|\mathbf{x}_{\mathrm{p}}\right\|=r_{\mathrm{p}}$. First, we use the proposed model for the analysis of the distribution of aggregate interference in infinite network. Then, we apply this method to capture the distribution of aggregate interference in a finite network. Although, finite network is a more realistic assumption in network deployments, no analytical tractable model exists for modeling and analysis of its performance metric.

\subsubsection{SIR and Coverage Probability}

Assume constant transmit power $P_{\mathrm{p}}$ for the transmitting node in the primary system. Let us assume that corresponding user is located at distance $r_{\mathrm{p}}$ away from the transmitter. This assumption is equivalent with the bipolar network model. We assume a standard power law path-loss function $l(\mathbf{x}-\mathbf{y})=\|\mathbf{x}-\mathbf{y}\|^{-\alpha}$ with path-loss exponent $\alpha>2$, and Rayleigh fading on all links from transmitters to the typical user, so that fading coefficients are independent exponential random variables with mean unity. Hence, the received power at a distance $r_{\mathrm{p}}$ from the transmitter is $P_{r}=P_{\mathrm{p}} h r_{\mathrm{p}}^{-\alpha}$, where $h \sim \exp (1)$ models Rayleigh fading. Without loss of generality, the analysis is performed at the typical user which is assumed to be at $\mathbf{x}_{\mathrm{p}}$. Then, for the transmitter located at $\mathbf{x} \in \Phi_{\mathbf{c}} \cap \mathbf{b}^{c}(0, D)$, let its transmit power be $P_{\mathrm{c}}$, denote the fading coefficient on the link to the typical user by $h_{\mathrm{x}}$, and assume that the path-loss exponent from all transmitters in $\Phi_{\mathrm{c}} \cap \mathbf{b}^{c}(0, D)$ is $\alpha$. Then the 
Chapter 5: Modeling and Analysis of Asymmetric Exclusion

total interference at the primary user from all interferers in $\Phi_{\mathrm{c}}$ is

$$
I=\sum_{\mathbf{x} \in \Phi_{\mathrm{c}} \cap \mathbf{b}^{c}(0, D)} P_{\mathrm{c}} h_{\mathbf{x}}\left\|\mathbf{x}-\mathbf{x}_{\mathrm{p}}\right\|^{-\alpha}
$$

We ignore thermal noise power at the typical user, i.e., we assume that the system is operating in the interference-limited regime. The resulting signal to interference ratio (SIR) expression can be expressed as:

$$
\operatorname{SIR}\left(r_{\mathrm{p}}\right)=\frac{P_{\mathrm{p}} h r_{\mathrm{p}}^{-\alpha}}{\sum_{\mathbf{x} \in \Phi_{\mathrm{c}} \cap \mathbf{b}^{c}(0, D)} P_{\mathrm{c}} h_{\mathbf{x}}\left\|\mathbf{x}-\mathbf{x}_{\mathrm{p}}\right\|^{-\alpha}} .
$$

Let $\gamma$ be the minimum SIR requirement for successful demodulation at the typical user. The typical node at the origin is in coverage if the SIR at the user exceeds the threshold $\gamma$. In other words, the SIR coverage probability $P_{\mathrm{c}}[9]$ is defined as follows

$$
\begin{aligned}
\mathrm{P}_{\mathrm{c}} & =\mathbb{P}\{\operatorname{SIR}(r)>\gamma\}=\mathbb{P}\left\{h>\frac{\gamma r_{\mathrm{p}}^{\alpha}}{P_{\mathrm{p}}} I\right\} \\
& =\mathbb{E}\left[\exp \left(-\frac{\gamma r_{\mathrm{p}}^{\alpha}}{P_{\mathrm{p}}} I\right)\right]=\mathcal{L}_{I}\left(\frac{\gamma r_{\mathrm{p}}^{\alpha}}{P_{\mathrm{p}}}\right)
\end{aligned}
$$

where the final equation is derived based on the definition of the Laplace transform coupled with the fact that the channel gain $h$ is an exponentially distributed random variable, $h \sim \exp (1)$. Here, $\mathcal{L}_{I}(s)=\mathbb{E}[\exp (-s I)]$ denotes the Laplace transform of the total interference $I$ from all interfering nodes. On the other hand, an outage occurs when the SIR falls below the threshold 
and so the outage probability is derived as follows

$$
\mathrm{P}_{\text {out }}=1-\mathrm{P}_{\mathrm{c}}
$$

\subsubsection{Asymmetric Exclusion Zone in Infinite Network}

Fig.5.1.a shows the system model which assumes an infinite network. Interference due to the secondary system outside the exclusion zone with radius $D$ provides the asymmetric interference field at the primary user. We propose an approach in which instead of removing an asymmetric exclusion zone of radius $D$, we model it by an inhomogeneous PPP. To this end, a virtual circular region centered at primary user with radius $D+r_{\mathrm{p}}$ is established. This region can be considered as a set of concentric thin rings of width $\mathrm{d} r$ centered at the origin with radius $r$. The total region between zero and $D-r_{\mathrm{p}}$ is located inside the exclusion zone of zero density and the density outside the zone with radius $D+r_{\mathrm{p}}$ is $\lambda_{\mathrm{c}}$. Thereby, the radius can change between $D-r_{\mathrm{p}}$ and $D+r_{\mathrm{p}}$. The total interference density inside the ring can be equivalently captured by the new density of $\lambda(r)$ using the transformation approach. Here,

$2 \pi r \lambda(r) \mathrm{d} r=2 r \mathrm{~d} r\left(\pi-\theta_{\mathrm{c}}(r)\right) \lambda_{\mathrm{c}}$, where the angle $\theta_{\mathrm{c}}(r)=\arccos \left(\frac{r^{2}+r_{\mathrm{p}}{ }^{2}-D^{2}}{2 r_{\mathrm{p}} r}\right)$ is defined in Fig.5.1.a.

Theorem 10 The Laplace transform of interference experienced by the primary user which is arbitrary located inside the exclusion zone by assuming 


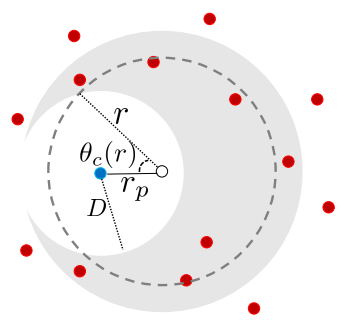

(a)

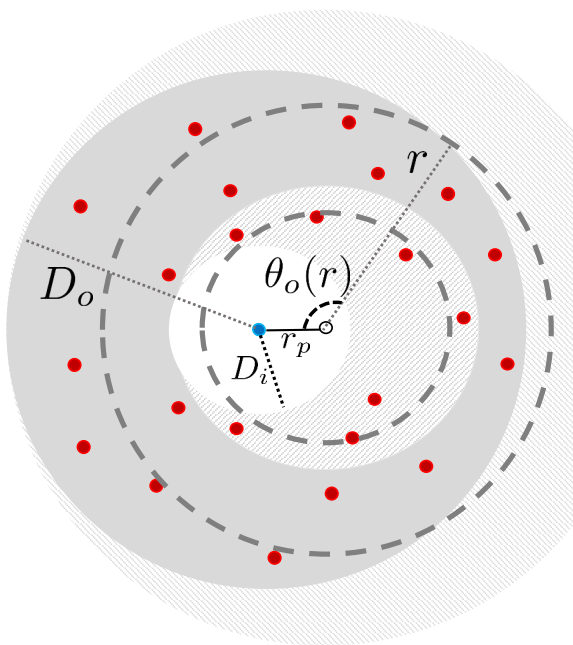

(b)

Figure 5.1: Modeling asymmetric exclusion zone (a) Infinite network; (b) Finite network.

that the network area be infinite is given by

$$
\mathcal{L}_{I}(s)=\exp \left(\int_{D-r_{\mathrm{p}}}^{D+r_{\mathrm{p}}} \frac{-2 \pi \lambda(r)}{1+\frac{r^{\alpha}}{P_{\mathrm{c}} s}} r \mathrm{~d} r-\int_{D+r_{\mathrm{p}}}^{\infty} \frac{2 \pi \lambda_{\mathrm{c}}}{1+\frac{r^{\alpha}}{P_{\mathrm{c}} s}} r \mathrm{~d} r\right)
$$

where $\lambda(r)=\left(1-\frac{\arccos \left(\frac{r^{2}+r_{\mathrm{p}}^{2}-D^{2}}{2 r_{\mathrm{p}} r}\right)}{\pi}\right) \lambda_{\mathrm{c}}$ the interference field, is infinite area outside the circular region with radius $D$.

Proof: See Appendix C.1.

\subsubsection{Asymmetric Exclusion Zone in Finite Network}

Fig. 5.1.b shows the system model in which the interference field is an annular region with inner radius of $D_{\mathrm{i}}$ and outer radius of $D_{\mathrm{o}}$. Here, interferes are drawn from $\Phi_{\mathrm{c}}$ with density $\lambda_{\mathrm{c}}$. The problem of asymmetric exclusion zone 
in finite network is studied in [80] by using a different approach and only for a special condition. In [80], a statistical model is derived by moment generating function (MGF) under specific path-loss exponent of $\alpha=2$ for asymmetric exclusion zone. Now by using our proposed approach, we can study this system model. First, we consider the virtual circular region with radius $D_{\mathrm{i}}+r_{\mathrm{p}}$ centered at $\mathbf{x}_{\mathrm{p}}$. By applying the proposed transformation, the interference distribution can be captured by an equivalent non-homogeneous density of $\lambda(r)$ between $D_{\mathrm{i}}-r_{\mathrm{p}}$ and $D_{\mathrm{i}}+r_{\mathrm{p}}$ as follows: $2 \pi r \lambda(r) \mathrm{d} r=2 r \mathrm{~d} r\left(\pi-\theta_{\mathrm{i}}(r)\right) \lambda_{\mathrm{c}}$ where the angle $\theta_{\mathrm{i}}(r)=\arccos \left(\frac{r^{2}+r_{\mathrm{p}}^{2}-D_{\mathrm{i}}^{2}}{2 r_{\mathrm{p}} r}\right)$. After capturing the impact of asymmetric interference in the first region, since the total region between $D_{\mathrm{i}}+r_{\mathrm{p}}$ and $D_{\mathrm{o}}-r_{\mathrm{p}}$ is located inside the interference field, the transformation in this region leads to the same density of $\lambda_{\mathrm{c}}$. Then, we derive the equivalence non-homogeneous density of $\lambda(r)$ corresponding to the region between $D_{\mathrm{o}}-r_{\mathrm{p}}$ and $D_{\mathrm{o}}+r_{\mathrm{p}}$ based on the same approach applied for the infinite network. It should be noted that in this case, the density of interferers corresponding to the thin rings of width $\mathrm{d} r$ inside the interference region is equal to $\lambda_{c}$ and outside the region is equal to zero. Here, the total interference density inside the ring can be captured by the new density of $\lambda(r)$ as follows: $2 \pi r \lambda(r) \mathrm{d} r=$ $2 r \mathrm{~d} r \theta_{\mathrm{o}}(r) \lambda_{\mathrm{c}}$ where the angle $\theta_{\mathrm{o}}(r)=\arccos \left(\frac{r^{2}+r_{\mathrm{p}}{ }^{2}-D_{\mathrm{o}}{ }^{2}}{2 r_{\mathrm{p}} r}\right)$. Using this result, we now derive the coverage probability of the primary user which is protected by an exclusion zone within a finite network while it is located arbitrary inside the network area. 
Theorem 11 The Laplace transform of interference experienced by the primary user which is arbitrary located inside the exclusion zone by assuming that the network area be finite is given by

$$
\begin{aligned}
& \mathcal{L}_{I}(s)= \\
& \exp \left(\int_{D_{\mathrm{i}}-r_{\mathrm{p}}}^{D_{\mathrm{i}}+r_{\mathrm{p}}} \frac{-2 \pi \lambda_{1}(r)}{1+\frac{r^{\alpha}}{P_{\mathrm{c}} s}} r \mathrm{~d} r-\int_{D_{\mathrm{i}}+r_{\mathrm{p}}}^{D_{\mathrm{o}}-r_{\mathrm{p}}} \frac{2 \pi \lambda}{1+\frac{r^{\alpha}}{P_{\mathrm{c}} s}} r \mathrm{~d} r-\int_{D_{\mathrm{o}}-r_{\mathrm{p}}}^{D_{\mathrm{o}}+r_{\mathrm{p}}} \frac{2 \pi \lambda_{2}(r)}{1+\frac{r^{\alpha}}{P_{\mathrm{c}} s}} r \mathrm{~d} r\right)
\end{aligned}
$$

where $\lambda_{1}(r)=\left(1-\frac{\arccos \left(\frac{r^{2}+r_{\mathrm{p}}{ }^{2}-D_{\mathrm{i}}{ }^{2}}{2 \mathrm{p}^{r}}\right)}{\pi}\right) \lambda_{\mathrm{c}}$ and $\lambda_{2}(r)=\left(\frac{\arccos \left(\frac{r^{2}+r_{\mathrm{p}}{ }^{2}-D_{\mathrm{o}}}{2 r_{\mathrm{p}} r}\right)}{\pi}\right) \lambda_{\mathrm{c}}$.

Proof: The proof follows on the same line as that of Theorem 10, except that interferers are distributed inside the finite region. Here, the interference field is the annular region with inner radius of $D_{\mathrm{i}}$ and outer radius of $D_{\mathrm{o}}$.

It should be noted that infinite network assumption can be derived as a special case of finite area network when $D_{\mathrm{o}} \rightarrow \infty$. Hence, we investigate the results corresponding to the finite area network which is more realistic and covers more scenarios including infinite network. Although by using the proposed approach, we have provided a tractable model for the analysis of the networks including the asymmetric exclusion zone scenario, the expressions involve a single integral. Now to further simplify the results, we derive closed-form expressions using the Taylor series expansion in the next section.

Closed form expression using Taylor series expansion. The expression ob- 
Corollary 1 Substituting the second order Taylor series into equation (5.7) for $\alpha=2$, we obtain

$\mathcal{L}_{I}(s ; 2)=$

$\frac{\sqrt{s}\left(-2 r_{\mathrm{p}} \sqrt{s}-\left({r_{\mathrm{p}}}^{2}-D_{\mathrm{k}}{ }^{2}-s\right) \arctan \left(\frac{2 r_{\mathrm{p}} \sqrt{s}}{-r_{\mathrm{p}}{ }^{2}+D_{\mathrm{k}}{ }^{2}+s}\right)\right)+r_{\mathrm{p}} \pi s \operatorname{arctanh}\left[\frac{2 r_{\mathrm{p}} D_{\mathrm{k}}}{{r_{\mathrm{p}}}^{2}+D_{\mathrm{k}}{ }^{2}+s}\right]}{2 r_{\mathrm{p}}}$

tained according to the proposed approach is readily computable but involves the integral expression. Using the second order of Taylor series expansion corresponds to $\arccos (\mathbf{x})$, i.e., $\arccos (\mathbf{x})=-\mathbf{x}+\frac{\pi}{2}$, we can obtain the closed form expression. It should be noted that by using the higher order of Taylor series expansion only the complexity of the integral increases while their impact on increasing the accuracy of the expression is negligible. Further, by using the second order of Taylor polynomials, the results are fairly accurate. We now find a closed form expression for the following common term corresponds to the coverage probability expression.

$$
\mathcal{L}_{I}(s ; \alpha)=\int_{D_{\mathrm{k}}-r_{\mathrm{p}}}^{D_{\mathrm{k}}+r_{\mathrm{p}}} \frac{\arccos \left(\frac{r^{2}+r_{\mathrm{p}}{ }^{2}-D_{\mathrm{k}}{ }^{2}}{2 r_{\mathrm{p}} r}\right)}{\pi\left(1+\frac{r^{\alpha}}{P_{\mathrm{c}} s}\right)} r \mathrm{~d} r
$$

For path-loss exponent values of $\alpha=\{2,3\}$, the closed form expressions are obtained in corollaries 1 and 2. Similarly, we can obtain closed form expressions for higher values of $\alpha$ such as $\alpha=4$.

In the next section, we study a cognitive network composed of the primary and secondary systems which operate on the same spectrum as a special case of interest. The major application of this system is IEEE 802.22 Digital TV 
Corollary 2 Substituting the second order Taylor series into equation (5.7) for $\alpha=3$, we obtain

$$
\begin{aligned}
\mathcal{L}_{I}(s ; 3) & =\frac{1}{12 r_{\mathrm{p}}} \sqrt[3]{s}\left(2 \sqrt{3}\left(-{r_{\mathrm{p}}}^{2}+D_{\mathrm{k}}^{2}+r_{\mathrm{p}} \pi \sqrt[3]{s}\right)\right. \\
& \times\left(\arctan \left[-\frac{\sqrt{3} r_{\mathrm{p}} \sqrt[3]{s}}{r_{\mathrm{p}}{ }^{2}-D_{\mathrm{k}}{ }^{2}+D_{k} \sqrt[3]{s}-s^{2 / 3}}\right]\right)+\left({r_{\mathrm{p}}}^{2}-D_{\mathrm{k}}{ }^{2}+r_{\mathrm{p}} \pi \sqrt[3]{s}\right) \\
& \times\left(2 \log \left[1-\frac{2 r_{\mathrm{p}}}{r_{\mathrm{p}}+D_{\mathrm{k}}+\sqrt[3]{s}}\right]+\log \left[\frac{\left(r_{\mathrm{p}}+D_{\mathrm{k}}\right)\left(r_{\mathrm{p}}+D_{\mathrm{k}}-\sqrt[3]{s}\right)+s^{2 / 3}}{\left(r_{\mathrm{p}}-D_{\mathrm{k}}\right)\left(r_{\mathrm{p}}-D_{\mathrm{k}}+\sqrt[3]{s}\right)+s^{2 / 3}}\right]\right) \\
& \left.+2 s^{2 / 3} \log \left[\frac{s-\left(r_{\mathrm{p}}-D_{\mathrm{k}}\right)^{3}}{s+\left(r_{\mathrm{p}}+D_{\mathrm{k}}\right)^{3}}\right]\right)
\end{aligned}
$$

Table 5.1: Network Parameters of Asymmetric Exclusion Zone

\begin{tabular}{l|l|l}
\hline \hline Symbol & Description & Default Value \\
\hline$\lambda_{\mathrm{c}}$ & Density of transmitters corresponding to $\Phi_{\mathrm{c}}$ & $100 \mathrm{~km}^{-2}$ \\
$\alpha$ & Path-loss exponent of system & - \\
$P_{\mathrm{c}}$ & Transmit power of interferers & $70 \mathrm{dBm}$ \\
$P_{\mathrm{p}}$ & Transmit power of primary transmitter & $80 \mathrm{dBm}$ \\
$r_{\mathrm{p}}$ & Serving distance corresponding to user & $0.2 \mathrm{~m}$ \\
$T$ & SIR threshold for successful reception on user & $-d B$ \\
$D_{\mathrm{i}}$ & Inner radius of interference field & $-m$ \\
$D_{\mathrm{o}}$ & Outer radius of interference field & $500 \mathrm{~m}$ \\
\hline \hline
\end{tabular}

which in the high power primary transmitter broadcasts signal to the user of interest (located at $r_{\mathrm{p}}$ ). The secondary transmitters inside the exclusion zone are inactive which leads to the protection of the primary system communication.

\subsection{Numerical Results and Discussion}

In this section, we present the numerical results for our proposed approach and validate our model against simulations. Unless otherwise specified, we use the default values of the network parameters as shown in Table 5.1, and 


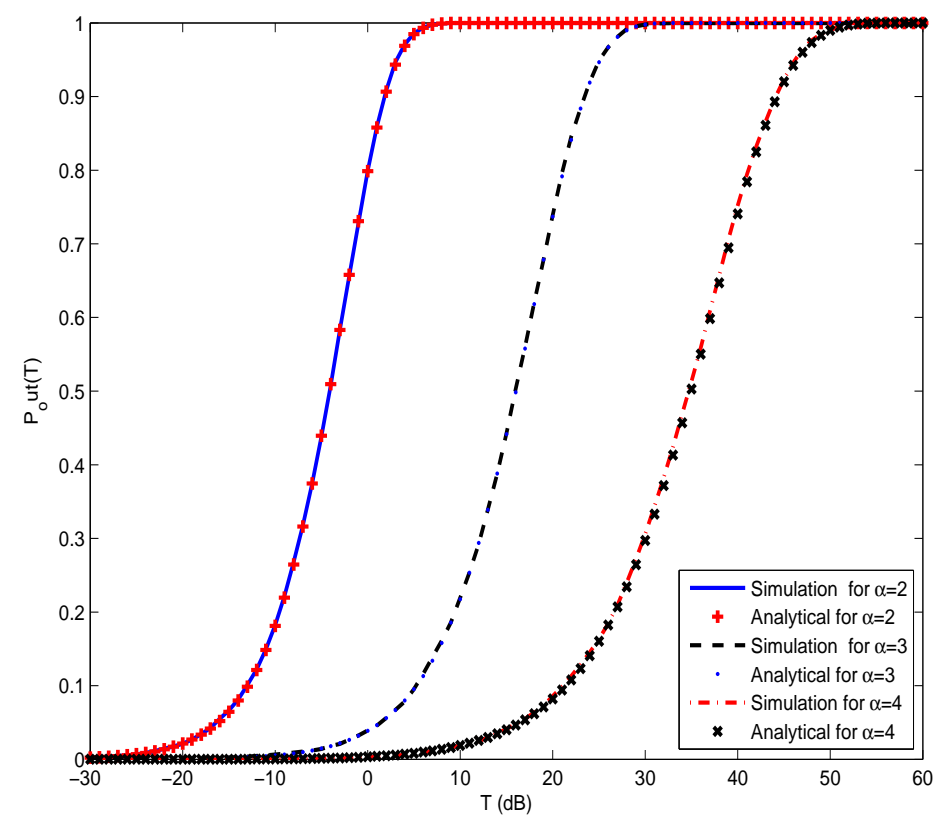

Figure 5.2: Comparison the outage probability of the PU between the second-order Taylor series expansion and simulation results; Setup: $R=$ $(20,25,500) m$

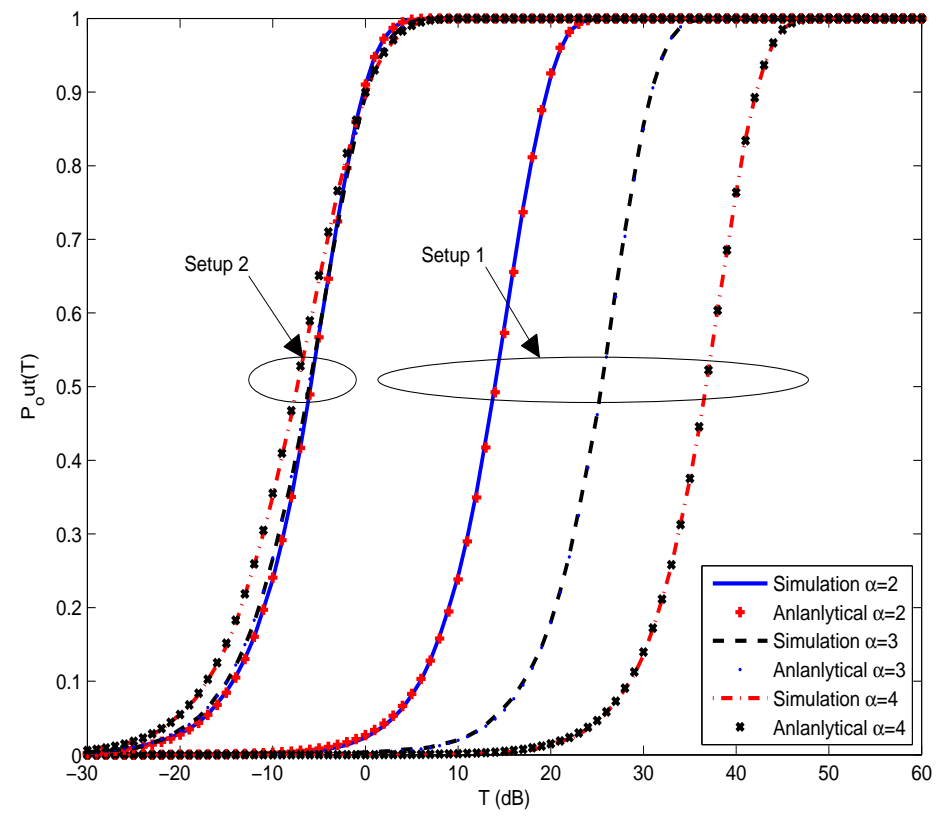

Figure 5.3: Impact of $\alpha$ on the outage probability of the user; Setup 1: $R=$ $(25,250,500) m$; Setup 2: $R=(200,250,500) m$ 


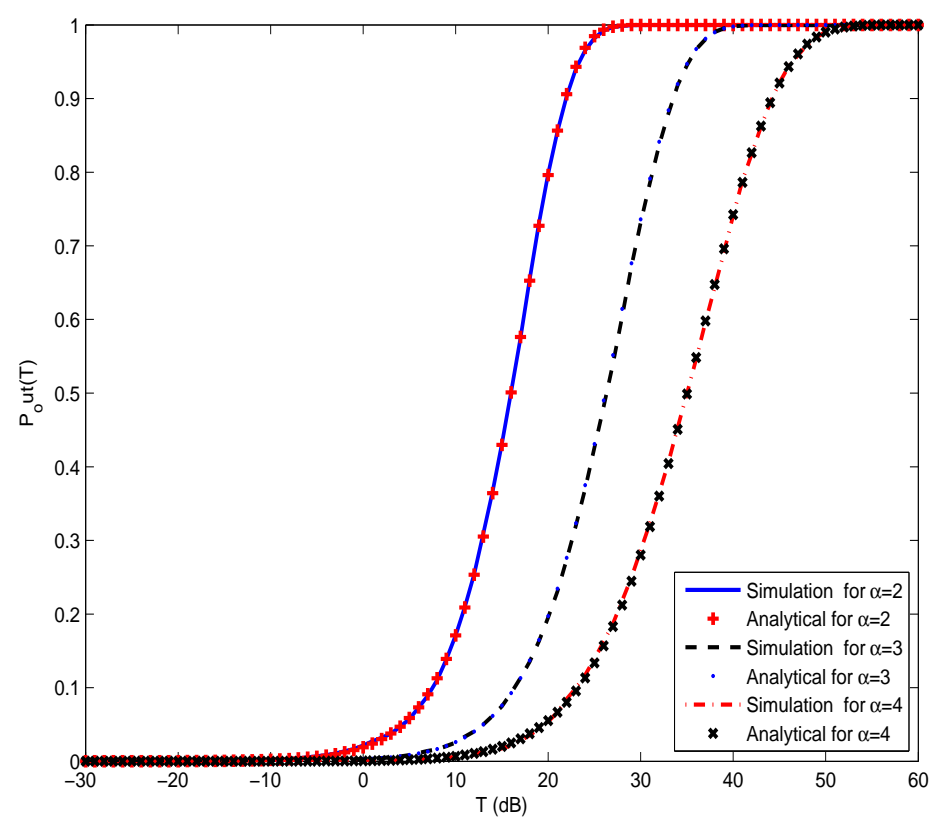

Figure 5.4: Impact of $\alpha$ on the outage of the PU; Setup: $R=(10,25,500) m$

$R=\left(r_{\mathrm{p}}, D_{\mathrm{i}}, D_{\mathrm{o}}\right) m$. All simulations are performed over an circular region with radius $D_{\mathrm{o}}=500 \mathrm{~m}$ and results are averaged over $10^{4}$ iterations. In each realization, the performance is evaluated for the typical user of the protected network at distance $r_{\mathrm{p}}$ from the origin while its transmitter is located at the origin. Fig. 5.2 investigates the accuracy of our closed-form analytical results using second order Taylor expansion series. Here, the outage probability of the system for different values of path-loss exponent is illustrated. As this figure shows, the analytical results which are obtained in closed form using the second order of Taylor expansion series are perfectly matched with simulation results. It should be noted that here the target received power at the typical user, is considered as a constant value of $50 \mathrm{dBm}$ and the transmit power of interferers is equal to $80 \mathrm{dBm}$. This figure not only validates the accuracy 
of the analytically derived expression based on the second order of Taylor expansion series but also shows the impact of different path-loss exponents on the outage probability of the user. Owing to the assumption of fixed target received power in this case, solely the interference powers change in resulting SIRs. In the setup corresponding to this figure, the inner exclusion zone starts from $D_{\mathrm{i}}=25 \mathrm{~m}$. Here, the user is located at $r_{\mathrm{p}}=20 \mathrm{~m}$ which is relatively close to the cell edge. By increasing the path-loss exponent from $\alpha=2$ to $\alpha=3$ and 4 , the outage probability decreases. In addition, the gaps between the outage probabilities for the various values of $\alpha$ are large.

Fig. 5.3 compares the outage probability of the analytical and simulation results for different values of $\alpha$ under an interference limited system. Two different setups are assumed in this figure such that in the both cases, the inner exclusion zone starts from $D_{\mathrm{i}}=250 \mathrm{~m}$ which is relatively large value and provides high protection against interference at the cost of inhibiting potential concurrent transmissions within the exclusion zone. In the first setup, the user is located at distance $r_{\mathrm{p}}=20 \mathrm{~m}$ from the origin while in the second one, it is at distance $r_{\mathrm{p}}=200 \mathrm{~m}$ from the origin which is nearby the cell edge. In this scenario, the received power can change as a function of standard path-loss exponent. An insightful observation from this figure is that for the lower path-loss exponents at secondary system, the user attains lower coverage probability. It is clear that in the first setup, the outage probability of the user which is close to its serving transmitter is significantly lower compared 
Chapter 5: Modeling and Analysis of Asymmetric ExClusion

to the second setup in which the user is nearby the cell edge. The impact of various values of $\alpha$ on the outage probability of the second setup is negligible and they are almost the same despite of changing the path-loss exponents. By increasing the radius of inner exclusion zone, interference works like noise and system shifts from the interference-limited system to the noise-limited one. Fig. 5.4 shows the outage probability of the primary user when the inner exclusion zone starts from $D_{\mathrm{i}}=25 \mathrm{~m}$. In this setup, the system is interference limited and also the target received power changes as a function of path-loss exponent. In comparison with Fig. 5.2, we observe that the gap between the outage probability of the primary user for different path-loss exponent is reduced. Furthermore, by reducing the inner radius of the interference field, the primary communication is less protected which leads to the higher outage probability. In general, the location of the typical user and the area of the exclusion zone provide design flexibility in achieving specified levels of outage probability and also other performance metrics such as spectral efficiency. 


\section{Chapter 6}

\section{Conclusion and Future Work}

\subsection{Summary}

In this work, we have focused on the modeling and analysis of interference management techniques in wireless networks. First, we have provided mathematical preliminaries corresponding to stochastic geometry tools for the modeling and analysis of wireless networks. Then, we have focused on the accurate performance characterization of a typical node in a wireless network that is modeled as the PHP. This model is of particular interest in scenarios where interference management techniques introduce spatial separation among active transmitters in the form of holes or exclusion zones which are areas with low interference field strength.

In terms of the technical results, we have provided new easy-to-use provable 
lower and upper bounds on the Laplace transform of interference experienced by a typical node in the PHP. In addition to accurately characterizing the interference power, these bounds immediately characterize the coverage probability of a typical node in the case where all the wireless links experience independent Rayleigh fading. Since the prior work has mostly focused on reducing the PHP to a PPP either by ignoring the holes or by matching the PPP density to that of the PHP, to the best of our knowledge, the proposed bounds are the tightest known bounds for the Laplace transform of interference in the PHP.

For the analysis, we proposed a new approach in which the holes are dissolved in such a way that the PHP is reduced to an equivalent (and more tractable) non-homogeneous PPP. The key in deriving tight bounds was to preserve the local neighborhood around the typical node while simplifying the far field to attain tractability. The tightness of the bounds is demonstrated analytically as well as numerically by comparing with simulations and known approaches.

Then, we have developed a new approach to the downlink coverage probability analysis of a two-tier HCN modeled as a PHP. The proposed approach is based on the tools developed for the analysis of PHPs in 3. The key idea is to curtail the complexity by preserving the local neighborhood of the interference field around the typical point while simplifying the far-field of interference. Using this approach, we derived a new and remarkably tight bound for the 
serving distance of a typical user in a PHP to its closest small cell.

We then derived coverage probability for a typical user under two closedaccess cases: (i) typical user is served by macro tier, and (ii) typical user is served by a small cell tier. Then we have focused on the modeling and analysis of asymmetric exclusion zones which can be formed by applying some types of interference management techniques in communication networks. One of the realistic scenarios in this field appears when an exclusion zone is established to protect the communication of the typical user located at an arbitrary location inside the cell. In this work, a general framework is provided by the proposed approach to facilitate the analysis and characterize the network performance clearly. This approach transforms the asymmetric interference field which is modeled using a homogeneous Poisson point process into a symmetric field of interfering nodes. We applied this approach for the modeling and analysis of the outage probability of the typical user surrounded by asymmetric exclusion zone. Using our transformation, the analytical tractable expressions which comprise only a single integral are derived. Further, using the Taylor series expansion, we have obtained the simplified closed form expressions which perfectly match the simulation results. 


\subsection{Future Direction}

Our proposed approach and results given by this work can be extended to study the following research problems.

\subsubsection{More general performance metrics and fading dis- tributions}

Our proposed approach in chapter 3 is not really limited to the analysis of Laplace transform of interference under Rayleigh fading. In principle, it can be used to study more general performance metrics that depend upon the received SIR under more general fading and shadowing distributions as well as general distance-dependent (isotropic) path-loss functions. To capture general fading distributions, $\kappa-\mu$ and $\eta-\mu$ fading models can be considered. These models are proposed in the literature [81] to represent environments with line of sight (LOS) or non-line-of-sight (NLOS) conditions. It should be noted that some other fading models such as Rician and Nakagami-m can be classified under these fading models.

Further, since the results given in this work determine the Laplace transform of SIR distribution can of course be used for the analysis of performance metrics such as coverage, rate and throughput. These results can be used for the analysis of the network throughput. Let us characterize the average number of bits successfully transmitted per unit time, per unit bandwidth, 
per unit area. This is termed as area spectral efficiency (ASE) and has units of bits $/ \mathrm{s} / \mathrm{Hz}$ per unit area. If there are $\lambda$ active transmitters per unit area achieving a coverage of $\operatorname{SIR}\left(r_{0}\right)>\gamma$ on the links to their respective receivers, then the ASE is

$$
\mathrm{ASE}=\lambda \mathbb{P}\left\{\operatorname{SIR}\left(r_{0}\right)>\gamma\right\} \log _{2}(1+\gamma)
$$

bits/s/Hz per unit area.

We have obtained tight bounds and approximation for $\mathbb{P}\left\{\operatorname{SIR}\left(r_{0}\right)>\gamma\right\}$, which directly enable the analysis of ASE. In other words, ASE is simple a scaled version of the coverage probability $\mathbb{P}\left\{\operatorname{SIR}\left(r_{0}\right)>\gamma\right\}$. Note that, the proposed approach can also be used to characterize other performance metrics of interest besides ASE. For instance, one can use it to study rate distribution, where rate is defined as: $\mathcal{R}=\log \left(1+\operatorname{SIR}\left(r_{0}\right)\right)$ bits/s/Hz. The distribution of $\mathcal{R}$ can be evaluated as:

$$
\begin{aligned}
\mathbb{P}\{\mathcal{R}>\rho\} & =\mathbb{P}\left(\log _{2}\left(1+\operatorname{SIR}\left(r_{0}\right)\right) \geq \rho\right) \\
& =\mathbb{P}\left(\operatorname{SIR}\left(r_{0}\right) \geq 2^{\rho}-1\right)
\end{aligned}
$$

where $\rho$ is rate threshold. Since we already know the coverage probability $\mathbb{P}\left\{\operatorname{SIR}\left(r_{0}\right)>\gamma\right\}$, just replacing $\gamma$ by $2^{\rho}-1$ gives us the rate distribution.

These results have numerous applications in a variety of wireless networks 
where interference management is performed by spatially separating the active links, such as in cognitive radio and D2D networks. Since our main emphasis was on characterization of the interference power, we assumed that the serving transmitter for the typical receiver is located at a fixed distance. This corresponds to an ad hoc network scenario. Relaxation and generalization of this assumption is a fruitful area of future investigation.

\subsubsection{Arbitrary shaped exclusion zones}

Another direction of future work is the extension of the current model to study exclusion zones with different shapes and sizes, e.g., circles with different radii. Transmitters which utilize dynamic interference mitigation techniques such as the one introduced in [48] change their request power as a function of received signal, thus leading to exclusion zones with dynamic radius.

In practical deployments, the shape of exclusion zones is not necessarily circular and interference management techniques can form exclusion zones with arbitrary shapes. Further, transmitters may not have permission to be inside some particular zones which are called forbidden zones and can create more general shapes. In comparison to the analysis of circular-shaped exclusion zones, the analysis of arbitrary-shaped exclusion zones is more challenging. One of the approaches for this analysis is based on approximating the exclusion zones by convex polygon regions. 
Then, the analysis of this setup is achievable based on the combination of approaches proposed in current work and the techniques used in [82] and [83].

\subsubsection{Modeling CSMA-based network}

The holes in the PHP are driven by a PPP. Extending this to other point processes, such as a Matérn process, is another promising direction of future work which has numerous applications in wireless networks. For instance, the impact of medium access mechanisms such as CSMA/CA with RTS/CTS in ad hoc networks is modeled in $[84,85]$. In [85], due to RTS/CTS mechanism, a protection zone is established around the receiver. It is the union of carrier sensing cleaned zone and RTS/CTS cleaned zone.

Two types of thinning according to Matérn hard core process are considered for this modeling. In the first one, a potential transmitter is kept when there is no other transmitter inside its exclusion zone. In the second one, a mark is assigned to each transmitter and a given transmitter is kept while there is no other transmitter with smaller mark inside its exclusion zone. The coexistence of unlicensed LTE and WiFi is one of the promising research directions. In [86], the coexistence of WiFi and small cells using unlicensed band is investigated. WiFi transmitters use CSMA protocol to access the channel. The coverage and throughput of the system is derived under the Matérn hard-core point process (HCPP) model. 


\subsubsection{Non-uniform user distribution and other applica-}

\section{tions}

The extensions toward characterizing the coverage probability of a typical user in an open access PHP-based HCN, and the non-uniform user distributions are promising future directions. In realistic small cell distributions, users are concentrated at certain areas in the cell, thereby forming hotspots. Another direction of future work is the extension of the current model to study a non uniform user distribution scenario in which the user and BS locations are correlated. In this scenario, the user locations can be modeled by a Poisson cluster process with the cluster centers being the BSs in a PHP-based HCN.

Further, this work can be extended for the analysis of primary users in a cognitive PHP-based ad-hoc network under two scenarios. First, the typical user is located at the center of the cell and second, exclusion zones are established around transmitter, while corresponding user is located at an arbitrary location inside the cell. Besides, the results given by chapter 4 can be used for the performance metrics such as coverage, rate and throughput of a PHPbased HCN. The possible extensions of the results in chapter 5 are numerous, and can appear in variety of wireless networks such as cognitive radio and device-to-device (D2D) networks. 


\subsection{List of Publications}

This dissertation is based on the following publications:

- Z. Yazdanshenasan, H. S. Dhillon, M. Afshang and P.H.J. Chong, "Poisson Hole Process: Theory and Applications to Wireless Networks," IEEE Trans. on Wireless Commun., Nov. 2016.

- Z. Yazdanshenasan, H. S. Dhillon and P.H.J. Chong, "Serving Distance and Coverage in a Closed Access PHP-Based Heterogeneous Cellular Network," in Proc. Biennial Symposium on Communications, Kelowna, British Columbia, June 2016.

- Z. Yazdanshenasan, H. S. Dhillon, M. Afshang and P.H.J. Chong, "Tight Bounds on the Laplace Transform of Interference in a Poisson Hole Process," IEEE ICC, Kuala Lumpur, Malaysia, May 2016.

- Z. Yazdanshenasan, M. Afshang, S. Mukherjee, and P.H.J. Chong, "Dynamic Interference Mitigation in Two tier HetNets: Modeling and Analysis," Proc. IEEE Vehicular Technology Conference (VTC), 2014.

- M. Afshang, Z. Yazdanshenasan, S. Mukherjee, and P.H.J. Chong, "Hybrid Division Duplex for HetNets: Coordinated Interference Management with Uplink Power Control," Proc., IEEE International Conference on Communications Workshops (ICC), 2015. 


\section{References}

[1] A. Ghosh, N. Mangalvedhe, R. Ratasuk, B. Mondal, M. Cudak, E. Visotsky, T. A. Thomas, J. G. Andrews, P. Xia, H. S. Jo, H. S. Dhillon, and T. D. Novlan, "Heterogeneous cellular networks: From theory to practice," IEEE Commun. Magazine, vol. 50, no. 6, pp. 54-64, Jun. 2012.

[2] J. G. Andrews, H. Claussen, M. Dohler, S. Rangan, and M. C. Reed, "Femtocells: Past, present, and future," IEEE Journal on Sel. Areas in Commun., vol. 30, no. 3, pp. 497-508, Apr. 2012.

[3] G. Zhang, T. Q. S. Quek, M. Kountouris, A. Huang, and H. Shan, "Fundamentals of heterogeneous backhaul design - analysis and optimization," IEEE Trans. on Commun., vol. 64, no. 2, pp. 876-889, Feb. 2016.

[4] W. C. Cheung, T. Q. S. Quek, and M. Kountouris, "Throughput optimization, spectrum allocation, and access control in two-tier femtocell networks," IEEE Journal on Sel. Areas in Commun., vol. 30, no. 3, pp. 561-574, Apr. 2012.

[5] T. D. Novlan, R. K. Ganti, A. Ghosh, and J. G. Andrews, "Analytical evaluation of fractional frequency reuse for OFDMA cellular networks," IEEE Trans. on Wireless Commun., vol. 10, no. 12, pp. 4294-4305, Dec. 2011.

[6] — - "Analytical evaluation of fractional frequency reuse for heterogeneous cellular networks," IEEE Trans. on Commun., vol. 60, no. 7, pp. 2029-2039, Jul. 2012.

[7] Z. Hasan, H. Boostanimehr, and V. K. Bhargava, "Green cellular networks: A survey, some research issues and challenges," IEEE Commun. Surveys and Tutorials, vol. 13, no. 4, pp. 524-540, Nov. 2011.

[8] M. Haenggi, J. G. Andrews, F. Baccelli, O. Dousse, and M. Franceschetti, "Stochastic geometry and random graphs for the analysis and design of wireless networks," IEEE Journal on Selected Areas in Commun., vol. 27, no. 7, pp. 1029-1046, Sep. 2009. 
[9] J. G. Andrews, F. Baccelli, and R. K. Ganti, "A tractable approach to coverage and rate in cellular networks," IEEE Trans. on Commun., vol. 59, no. 11, pp. 3122-3134, Nov. 2011.

[10] D. López-Pérez, A. Valcarce, G. De La Roche, and J. Zhang, "OFDMA femtocells: A roadmap on interference avoidance," IEEE Commun. Magazine, vol. 47, no. 9, pp. 41-48, Sep. 2009.

[11] G. Nigam, P. Minero, and M. Haenggi, "Spatiotemporal cooperation in heterogeneous cellular networks," IEEE Journal on Sel. Areas in Commun., vol. 33, no. 6, pp. 1253-1265, Jun. 2015.

[12] S. Samarakoon, M. Bennis, W. Saad, and M. Latva-Aho, "Enabling relaying over heterogeneous backhauls in the uplink of femtocell networks," in Proc., IEEE Intl. Symposium on Modeling and Optimization in Mobile, Ad Hoc and Wireless Networks (WiOpt), May 2012.

[13] H. Holma and A. Toskala, LTE for UMTS: Evolution to LTE-advanced. John Wiley \& Sons, 2011.

[14] P. Omiyi, H. Haas, and G. Auer, "Analysis of TDD cellular interference mitigation using busy-bursts," IEEE Trans. on Wireless Commun., vol. 6, no. 7, pp. 2721-2731, Jul. 2007.

[15] S.-M. Cheng, S.-Y. Lien, F.-S. Chu, and K.-C. Chen, "On exploiting cognitive radio to mitigate interference in macro/femto heterogeneous networks," IEEE Trans. on Wireless Commun., vol. 18, no. 3, pp. 40-47, Jun. 2011.

[16] H. ElSawy, E. Hossain, and M.-S. Alouini, "Analytical modeling of mode selection and power control for underlay D2D communication in cellular networks," IEEE Trans. on Wireless Commun., vol. 62, no. 11, pp. 41474161, Nov. 2014.

[17] Y. Sun, R. P. Jover, and X. Wang, "Uplink interference mitigation for OFDMA femtocell networks," IEEE Trans. on Wireless Commun., vol. 11, no. 2, pp. 614-625, Feb. 2012.

[18] L. Giupponi and C. Ibars, "Distributed interference control in OFDMAbased femtocells," in Proc., IEEE Intl. Symposium on Personal Indoor and Mobile Radio Commun. (PIMRC), Sep. 2010.

[19] H. Zhang, X. Chu, W. Zheng, and X. Wen, "Interference-aware resource allocation in co-channel deployment of OFDMA femtocells," in Proc., IEEE Intl. Conf. on Commun. (ICC), Jun. 2012. 
[20] X. Kang, R. Zhang, and M. Motani, "Price-based resource allocation for spectrum-sharing femtocell networks: A stackelberg game approach," IEEE Journal on Sel. Areas in Commun., vol. 30, no. 3, pp. 538-549, Apr. 2012.

[21] R. Xie, F. R. Yu, H. Ji, and Y. Li, "Energy-efficient resource allocation for heterogeneous cognitive radio networks with femtocells," IEEE Trans. on Wireless Commun., vol. 11, no. 11, pp. 3910-3920, Nov. 2012.

[22] O. N. Gharehshiran, A. Attar, and V. Krishnamurthy, "Collaborative sub-channel allocation in cognitive LTE femto-cells: A cooperative gametheoretic approach," IEEE Trans. on Commun., vol. 61, no. 1, pp. 325334, Jan. 2013.

[23] J. Venkataraman, M. Haenggi, and O. Collins, "Shot noise models for outage and throughput analyses in wireless ad hoc networks," in Proc., IEEE Military Commun. conference (MILCOM), Oct. 2006.

[24] M. Z. Win, P. C. Pinto, and L. A. Shepp, "A mathematical theory of network interference and its applications," Proc. IEEE, vol. 97, no. 2, pp. 205-230, Feb. 2009.

[25] A. Hasan and J. G. Andrews, "The guard zone in wireless ad hoc networks," IEEE Trans. on Wireless Commun., vol. 6, no. 3, pp. 897-906, Mar. 2007.

[26] D. Torrieri and M. C. Valenti, "Exclusion and guard zones in DS-CDMA ad hoc networks," IEEE Trans. on Commun., vol. 61, no. 6, pp. 24682476, Jun. 2013.

[27] G. Alfano, M. Garetto, and E. Leonardi, "New insights into the stochastic geometry analysis of dense CSMA networks," in Proc., IEEE INFOCOM, Apr. 2011.

[28] X. Zhang and M. Haenggi, "Random power control in Poisson networks," IEEE Trans. on Commun., vol. 60, no. 9, pp. 2602-2611, Sep. 2012.

[29] J. Lee, J. G. Andrews, and D. Hong, "Spectrum-sharing transmission capacity with interference cancellation," IEEE Trans. on Commun., vol. 61, no. 1, pp. 76-86, Jan. 2013.

[30] C. h. Lee and M. Haenggi, "Interference and outage in Poisson cognitive networks," IEEE Trans. on Wireless Commun., vol. 11, no. 4, pp. 13921401, Apr. 2012.

[31] H. ElSawy and E. Hossain, "A modified hard core point process for analysis of random CSMA wireless networks in general fading environments," IEEE Trans. on Commun., vol. 61, no. 4, pp. 1520-1534, Apr. 2013. 
[32] Z. Gong and M. Haenggi, "Interference and outage in mobile random networks: Expectation, distribution, and correlation," IEEE Trans. on Mobile Computing, vol. 13, no. 2, pp. 337-349, Feb. 2014.

[33] C. H. de Lima, M. Bennis, and M. Latva-aho, "Coordination mechanisms for self-organizing femtocells in two-tier coexistence scenarios," IEEE Trans. on Wireless Commun., vol. 11, no. 6, pp. 2212-2223, Feb. 2012.

[34] H. ElSawy and E. Hossain, "Two-tier HetNets with cognitive femtocells: Downlink performance modeling and analysis in a multichannel environment," IEEE Trans. on Mobile Computing., vol. 13, no. 3, pp. 649-663, Mar. 2014.

[35] S.-M. Cheng, W. C. Ao, F.-M. Tseng, and K.-C. Chen, "Design and analysis of downlink spectrum sharing in two-tier cognitive femto networks," IEEE Trans. on Vehicular Technology., vol. 61, no. 5, pp. 2194-2207, Jun. 2012.

[36] H.-S. Jo, Y. J. Sang, P. Xia, and J. G. Andrews, "Heterogeneous cellular networks with flexible cell association: A comprehensive downlink SINR analysis," IEEE Trans. on Wireless Commun., vol. 11, no. 10, pp. 34843495, Oct. 2012.

[37] A. Adhikary, V. Ntranos, and G. Caire, "Cognitive femtocells: Breaking the spatial reuse barrier of cellular systems," in Proc. IEEE Information Theory and Applications Workshop (ITA), Feb. 2011.

[38] Y. Zhong and W. Zhang, "Multi-channel hybrid access femtocells: A stochastic geometric analysis," IEEE Trans. on Commun., vol. 61, no. 7, pp. 3016-3026, Jul. 2013.

[39] R. W. Heath, M. Kountouris, and T. Bai, "Modeling heterogeneous network interference using Poisson point processes," in IEEE Trans. on Signal Process., Aug. 2012.

[40] Y. S. Soh, T. Q. S. Quek, M. Kountouris, and G. Caire, "Cognitive hybrid division duplex for two-tier femtocell networks," IEEE Trans. on Wireless Commun., vol. 12, no. 10, pp. 4852-4865, Oct. 2013.

[41] Y. S. Soh, T. Q. Quek, M. Kountouris, and G. Caire, "Flexible duplex for cognitive femtocells in two-tier networks," in Proc., IEEE Globecom Workshops, Dec. 2012.

[42] T. D. Novlan, H. S. Dhillon, and J. G. Andrews, "Analytical modeling of uplink cellular networks," IEEE Trans. on Wireless Commun, vol. 12, no. 6, pp. 2669-2679, Jun. 2013. 
[43] F. J. Martin-Vega, F. J. Lopez-Martinez, G. Gómez, and M. C. AguayoTorres, "Multi-user coverage probability of uplink cellular systems: A stochastic geometry approach," in Proc. IEEE Global Commun. Conference, Dec. 2014.

[44] H. Elsawy and E. Hossain, "On stochastic geometry modeling of cellular uplink transmission with truncated channel inversion power control," IEEE Trans. on Commun., vol. 13, no. 8, pp. 4454-4469, Aug. 2014.

[45] F. Baccelli and A. Giovanidis, "A stochastic geometry framework for analyzing pairwise-cooperative cellular networks," IEEE Trans. on Wireless Commun., Feb. 2014.

[46] N. Deng, W. Zhou, and M. Haenggi, "A heterogeneous cellular network model with inter-tier dependence," in Proc., IEEE Globecom Workshops, Dec. 2014.

[47] — , "Heterogeneous cellular network models with dependence," IEEE Journal on Sel. Areas in Commun., vol. 33, no. 10, pp. 2167-2181, Oct. 2015 .

[48] M. Afshang, Z. Yazdanshenasan, S. Mukherjee, and P. H. J. Chong, "Hybrid division duplex for HetNets: Coordinated interference management with uplink power control," in Proc., IEEE Intl. Conference on Commun. Workshops (ICC), Jun. 2015.

[49] H. Sun, M. Wildemeersch, M. Sheng, and T. Q. Quek, "D2D enhanced heterogeneous cellular networks with dynamic TDD," IEEE Trans. on Wireless Commun., vol. 14, no. 8, pp. 4204-4218, Aug. 2015.

[50] A. H. Sakr and E. Hossain, "Cognitive and energy harvesting-based D2D communication in cellular networks: Stochastic geometry modeling and analysis," IEEE Trans. on Commun., vol. 63, no. 5, pp. 1867-1880, May 2015.

[51] M. Afshang and H. S. Dhillon, "Spatial modeling of device-to-device networks: Poisson cluster process meets Poisson hole process," to appear in Proc. Asilomar, Pacific Grove, CA, Nov. 2015.

[52] C. Cordeiro, K. Challapali, D. Birru, and N. Sai Shankar, "IEEE 802.22: The first worldwide wireless standard based on cognitive radios," in IEEE International Symposium, Nov. 2005.

[53] M. Vu, N. Devroye, and V. Tarokh, "The primary exclusive region in cognitive networks," in Proc., IEEE Consumer Commun. and Networking Conference (CCNC), Jan. 2008. 
[54] P. Madhusudhanan, Y. Liu, and T. Brown, "On primary user coverage probabilities and faulty cognitive radios," IEEE Trans. on Wireless Commun., vol. 13, no. 11, pp. 6207-6218, Nov. 2014.

[55] H. Q. Nguyen, F. Baccelli, and D. Kofman, "A stochastic geometry analysis of dense IEEE 802.11 networks," in Proc., IEEE INFOCOM, May 2007.

[56] M. Kaynia, N. Jindal, and G. E. Oien, "Improving the performance of wireless ad hoc networks through MAC layer design," IEEE Trans. on Wireless Commun., vol. 10, no. 1, pp. 240-252, Jan. 2011.

[57] H. ElSawy and E. Hossain, "Two-tier HetNets with cognitive femtocells: Downlink performance modeling and analysis in a multichannel environment," IEEE Trans. on Mobile Computing., vol. 13, no. 3, pp. 649-663, Mar. 2014.

[58] Z. Chen, C.-X. Wang, X. Hong, J. S. Thompson, S. A. Vorobyov, X. Ge, H. Xiao, and F. Zhao, "Aggregate interference modeling in cognitive radio networks with power and contention control," IEEE Trans. on Commun., vol. 60 , no. 2, pp. 456-468, Feb. 2012.

[59] E. Salbaroli and A. Zanella, "Interference analysis in a Poisson field of nodes of finite area," IEEE Trans. on Vehicular Technology., vol. 58, no. 4, pp. 1776-1783, Jul. 2009.

[60] D. Torrieri and M. C. Valenti, "The outage probability of a finite ad hoc network in Nakagami fading," IEEE Trans. on Commun., vol. 60, no. 11, pp. 3509-3518, Nov. 2012.

[61] Z. Yazdanshenasan, H. S. Dhillon, M. Afshang, and P. H. J. Chong, "Poisson hole process: Theory and applications to wireless networks," IEEE Trans. on Wireless Commun., vol. 15, no. 11, pp. 7531-7546, Nov. 2016.

[62] — - "Tight bounds on the Laplace transform of interference in a Poisson hole process," IEEE ICC, Kuala Lumpur, Malaysia, May 2016.

[63] D. H. S. Yazdanshenasan, Zeinab and P. H. J. Chong, "Serving distance and coverage in a closed access PHP-based Heterogeneous cellular network," in Proc. Biennial Symposium on Commun., British Columbia, Jun. 2016.

[64] J. Xu, J. Zhang, and J. G. Andrews, "On the accuracy of the Wyner model in cellular networks," IEEE Trans. on Wireless Commun,, vol. 10, no. 9, pp. 3098-3109, Sep. 2011. 
[65] D. Stoyan, W. Kendall, and J. Mecke, "Stochastic geometry and its applications, 1995," Akademie-Verlag, Berlin.

[66] O. Kallenberg, Foundations of modern probability. springer, 2002.

[67] M. Haenggi, Stochastic geometry for wireless networks. Cambridge University Press, 2012.

[68] H. ElSawy, E. Hossain, and M. Haenggi, "Stochastic geometry for modeling, analysis, and design of multi-tier and cognitive cellular wireless networks: A survey," IEEE Commun. Surveys and Tutorials, vol. 15, no. 3, pp. 996-1019, Mar. 2013.

[69] S. Weber, J. G. Andrews, and N. Jindal, "An overview of the transmission capacity of wireless networks," IEEE Trans. on Commun, vol. 58, no. 12, pp. 3593-3604, Dec. 2010.

[70] M. Haenggi and R. K. Ganti, Interference in large wireless networks. Now Publishers Inc, 2009.

[71] S. N. Chiu, D. Stoyan, W. S. Kendall, and J. Mecke, Stochastic Geometry and its Applications. John Wiley \& Sons, 2013.

[72] F. Baccelli and B. Blaszczyszyn, Stochastic Geometry and Wireless Networks: Volume 1: Theory. Now Publishers Inc, 2009, vol. 1.

[73] H. S. Dhillon, R. K. Ganti, F. Baccelli, and J. G. Andrews, "Modeling and analysis of K-tier downlink heterogeneous cellular networks," IEEE Journal on Sel. Areas in Commun., vol. 30, no. 3, pp. 550-560, Apr. 2012.

[74] S. Mukherjee, "Distribution of downlink SINR in heterogeneous cellular networks," IEEE Journal on Sel. Areas in Commun., vol. 30, no. 3, pp. 575-585, Apr. 2012.

[75] R. K. Ganti and M. Haenggi, "Regularity in sensor networks," in Proc., Intl. Zurich Seminar on Commun., Jul. 2006.

[76] M. Afshang, H. Dhillon, and P. Chong, "Modeling and performance analysis of clustered device-to-device networks," IEEE Trans. on Wireless Commun., vol. 15, no. 7, pp. 4957-4972, Apr. 2016.

[77] M. Afshang, H. S. Dhillon, and P. H. J. Chong, "Coverage and area spectral efficiency of clustered device-to-device networks," in Proc. IEEE Globecom, San Deigo, Dec. 2015.

[78] M. Haenggi, "On distances in uniformly random networks," IEEE Trans. on Info. Theory, vol. 51, no. 10, pp. 3584-3586, Oct. 2005. 
[79] E. U. T. R. Access, "Further advancements for E-UTRA physical layer aspects," 3GPP TR 36.814, Tech. Rep., 2010.

[80] L. Vijayandran, P. Dharmawansa, T. Ekman, and C. Tellambura, "Analysis of aggregate interference and primary system performance in finite area cognitive radio networks," IEEE Trans. on Commun., vol. 60, no. 7, pp. 1811-1822, Jul. 2012.

[81] M. D. Yacoub, "The $\kappa-\mu$ distribution and the $\eta-\mu$ distribution," IEEE Antennas Propag. Mag., vol. 49, no. 1, pp. 68-81, Feb. 2007.

[82] J. Guo, S. Durrani, and X. Zhou, "Outage probability in arbitrarilyshaped finite wireless networks," IEEE Trans. on Commun., vol. 62, no. 2, pp. 699-712, Feb. 2014.

[83] Z. Khalid and S. Durrani, "Distance distributions in regular polygons," IEEE Trans. on Vehicular Technology, vol. 62, no. 5, pp. 2363-2368, Jun. 2013.

[84] F. Baccelli, J. Li, T. Richardson, S. Shakkottai, S. Subramanian, and $\mathrm{X}$. Wu, "On optimizing CSMA for wide area ad hoc networks," Springer Queueing Systems, vol. 72, no. 1-2, pp. 31-68, May 2012.

[85] Y. Zhong, W. Zhang, and M. Haenggi, "Stochastic analysis of the mean interference for the RTS/CTS mechanism," in Proc., IEEE Intl. Conference on Commun. (ICC), Jun. 2014.

[86] X. Ding, C. H. Liu, L. C. Wang, and X. Zhao, "Coexisting success probability and throughput of multi-RAT wireless networks with unlicensed band access," IEEE Letters on Wireless Commun., vol. 5, no. 1, pp. 4-7, Feb. 2016.

[87] J. Roe, Elementary geometry. Oxford University Press, 1993. 


\section{Appendix A}

\section{A.1 Proof of Lemma 1}

In this case, the PHP $\Psi$ is approximated by the baseline $\operatorname{PPP} \Phi_{2}$, which reduces the problem to the well-studied problem of deriving Laplace transform of interference originating from the PPP field of interferers [67]. For completeness, the sketch of the derivation is provided next.

$$
\begin{aligned}
\mathcal{L}_{I}(s) & \geq \mathbb{E}\left[\exp \left(-s \sum_{\mathbf{x} \in \Phi_{2}} P h_{\mathbf{x}}\|\mathbf{x}\|^{-\alpha}\right)\right] \\
& \stackrel{(\mathrm{a})}{=} \exp \left(-\lambda_{2} \int_{\mathbb{R}^{2}} 1-\mathbb{E}_{h_{\mathbf{x}}}\left[\exp \left(-s P h_{\mathbf{x}}\|\mathbf{x}\|^{-\alpha}\right)\right] \mathrm{d} \mathbf{x}\right) \\
& \stackrel{(\mathrm{b})}{=} \exp \left(-\lambda_{2} \int_{\mathbb{R}^{2}} \frac{1}{1+\frac{\|\mathbf{x}\|^{\alpha}}{s P}} \mathrm{~d} \mathbf{x}\right) \stackrel{(\mathrm{c})}{=} \exp \left[-\pi \lambda_{2} \frac{(s P)^{2 / \alpha}}{\operatorname{sinc}(2 / \alpha)}\right]
\end{aligned}
$$

where (a) follows from the PGFL of a PPP [71], (b) from $h_{\mathbf{x}} \sim \exp (1)$, and (c) using standard machinery, where the integral is first converted form Cartesian to polar coordinates and the closed form expression follows from the properties of the Gamma function [73, Appendix B]. 


\section{A.2 Proof of Lemma 5}

The Laplace transform of interference conditioned on the distance of the hole center to the origin, $\|\mathbf{y}\|$, is

$$
\begin{aligned}
& \mathcal{L}_{I \mid\|\mathbf{y}\|}(s)=\mathbb{E}\left[\exp \left(-s \sum_{\left.\mathbf{x} \in \Phi_{2} \cap \mathbf{b}^{c}(\mathbf{y}, D)\right)} P h_{\mathbf{x}}\|\mathbf{x}\|^{-\alpha}\right)\right] \\
& =\mathbb{E}_{\Phi_{2}}\left[\prod_{\left.\mathbf{x} \in \Phi_{2} \cap \mathbf{b}^{c}(\mathbf{y}, D)\right)} \mathbb{E}_{h_{\mathbf{x}}}\left[\exp \left(-s P h_{\mathbf{x}}\|\mathbf{x}\|^{-\alpha}\right)\right]\right] \\
& =\mathbb{E}_{\Phi_{2}}\left[\prod_{\left.\mathbf{x} \in \Phi_{2} \cap \mathbf{b}^{c}(\mathbf{y}, D)\right)} \frac{1}{1+s P\|\mathbf{x}\|^{-\alpha}}\right] \stackrel{(\mathrm{a})}{=} \exp \left(-\lambda_{2} \int_{\mathbb{R}^{2} \backslash \mathcal{C}} \frac{1}{1+\frac{\|\mathbf{x}\|^{\alpha}}{s P}} \mathrm{~d} \mathbf{x}\right) \\
& =\exp \left(-\lambda_{2}\left(\int_{\mathbb{R}^{2}} \frac{1}{1+\frac{\|\mathbf{x}\|^{\alpha}}{s P}} \mathrm{~d} \mathbf{x}-\int_{\mathbf{b}(\mathbf{y}, D)} \frac{1}{1+\frac{\|\mathbf{x}\|^{\alpha}}{s P}} \mathrm{~d} \mathbf{x}\right)\right) \\
& \stackrel{(\mathrm{b})}{=} \exp \left(-\pi \lambda_{2} \frac{(s P)^{2 / \alpha}}{\operatorname{sinc}(2 / \alpha)}\right) \times \exp \left(2 \lambda_{2} \int_{\|\mathbf{y}\|-D}^{\|\mathbf{y}\|+D} \frac{\arccos \left(\frac{r^{2}+\|\mathbf{y}\|^{2}-D^{2}}{2\|\mathbf{y}\| r}\right)}{1+\frac{r^{\alpha}}{s P}} r \mathrm{~d} r\right)
\end{aligned}
$$

where (a) follows from the expression for the PGFL of a PPP and (b) is derived by the standard machinery, where the integral is first converted form Cartesian to polar coordinates and the closed form expression is then derived by using the properties of the Gamma function (in the same way as step (c) in the proof of Lemma 1 in Appendix A.1) [73, Appendix B]. The second term follows from the cosine-law: $r^{2}+\|\mathbf{y}\|^{2}-2 r\|\mathbf{y}\| \cos \theta(r)=D^{2}$ (Fig. 3.1).

By substituting $\lambda(r)=\frac{\lambda_{2}}{\pi} \arccos \left(\frac{r^{2}+\|\mathbf{y}\|^{2}-D^{2}}{2\|\mathbf{y}\| r}\right)$, the final expression in equation (3.10), is derived. 


\section{A.3 Proof of Theorem 2}

The lower bound on the Laplace transform of interference is

$$
\begin{aligned}
\mathcal{L}_{I}(s) & \geq \mathbb{E}\left[\exp \left(-s \sum_{\mathbf{x} \in \Phi_{2} \cap \mathbf{b}^{c}\left(\mathbf{y}_{1}, D\right)} P h_{\mathbf{x}}\|\mathbf{x}\|^{-\alpha}\right)\right] \\
& =\int_{D}^{\infty} \mathcal{L}_{I \mid\left\|\mathbf{y}_{1}\right\|}(s ; \lambda, D) f_{V_{1}}\left(v_{1}\right) \mathrm{d} v_{1} \stackrel{(\mathrm{a})}{=} \exp \left(-\pi \lambda_{2} \frac{(s P)^{2 / \alpha}}{\operatorname{sinc}(2 / \alpha)}\right) \\
& \times \int_{D}^{\infty} \exp \left(\int_{v_{1}-D}^{v_{1}+D} 2 \lambda_{2} \frac{\arccos \left(\frac{r^{2}+v_{1}^{2}-D^{2}}{2 v_{1} r}\right)}{1+\frac{r^{\alpha}}{s P}} r \mathrm{~d} r\right) \\
& \times 2 \pi \lambda_{1} v_{1} \exp \left(-\pi \lambda_{1}\left(v_{1}^{2}-D^{2}\right)\right) \mathrm{d} v_{1}=\exp \left(-\pi \lambda_{2} \frac{(s P)^{2 / \alpha}}{\operatorname{sinc}(2 / \alpha)}\right) \\
& \times \int_{D}^{\infty} \exp \left(f\left(v_{1}\right)\right) 2 \pi \lambda_{1} v_{1} \exp \left(-\pi \lambda_{1}\left(v_{1}^{2}-D^{2}\right)\right) \mathrm{d} v_{1}
\end{aligned}
$$

where $\mathbf{b}\left(\mathbf{y}_{1}, D\right)$ denotes the exclusion zone centered at $\mathbf{y}_{1}$ with radius $D$, and

(a) follows by substituting the conditional Laplace transform expression from

Lemma 5, and the PDF of $V_{1}$ from (4.3), $f\left(v_{1}\right)=\int_{v_{1}-D}^{v_{1}+D} \arccos \left(\frac{r^{2}+v_{1}^{2}-D^{2}}{2 v_{1} r}\right) \frac{2 \lambda_{2}}{1+\frac{r \alpha}{P s}} r \mathrm{~d} r$.

\section{A.4 Proof of Theorem 3}

By definition, the Laplace transform of the PHP is

$$
\begin{aligned}
& \mathcal{L}_{I}(s) \stackrel{(\mathrm{a})}{=} \mathbb{E}\left[\exp \left(-s \sum_{\mathbf{x} \in \Phi_{2} \cap \Xi_{D}^{c}} P h_{\mathbf{x}}\|\mathbf{x}\|^{-\alpha}\right)\right] \\
& \stackrel{(\mathrm{b})}{=} \mathbb{E}_{\Phi_{1}}\left[\exp \left(-\lambda_{2}\left(\int_{\mathbb{R}^{2}} \frac{\mathrm{d} \mathbf{x}}{1+\frac{\|\mathbf{x}\|^{\alpha}}{s P}}-\int_{\Xi_{D}} \frac{\mathrm{d} \mathbf{x}}{1+\frac{\|\mathbf{x}\|^{\alpha}}{s P}}\right)\right)\right]
\end{aligned}
$$


where $\Xi_{D}$ in (a) is $\triangleq \bigcup_{\mathbf{y} \in \Phi_{1}} \mathbf{b}(\mathbf{y}, D)$ as defined in (3.1), (b) follows by taking expectations over channel gains $h_{\mathbf{x}} \sim \exp (1)$ and the $\operatorname{PPP} \Phi_{2}$ given $\Xi_{D}$, where we use the PGFL of a PPP to take expectation over $\Phi_{2}$. Note the integral over $\Xi_{D}$ is not easy to compute due to the possible overlaps in the holes. Therefore, to derive the bound, we use

$$
\int_{\Xi_{D}} \frac{\mathrm{d} \mathbf{x}}{1+\frac{\|\mathbf{x}\|^{\alpha}}{s P}} \leq \sum_{\mathbf{y} \in \Phi_{1}} \int_{\mathbf{b}(\mathbf{y}, D)} \frac{\mathrm{d} \mathbf{x}}{1+\frac{\|\mathbf{x}\|^{\alpha}}{s P}}
$$

which follows by ignoring the effect of overlaps. Substituting this back in the expression of $\mathcal{L}_{I}(s)$; solving the first integral as done in Lemma 5; and using the result of Lemma 5 to handle the integral over $\mathbf{b}(\mathbf{y}, D)$, we get

$$
\begin{aligned}
\mathcal{L}_{I}(s) & \leq \exp \left(-\pi \lambda_{2} \frac{(s P)^{2 / \alpha}}{\operatorname{sinc}(2 / \alpha)}\right) \\
& \times \mathbb{E}_{\Phi_{1}}\left[\prod_{\mathbf{y} \in \Phi_{1}} \exp \left(2 \lambda_{2} \int_{\|\mathbf{y}\|-D}^{\|\mathbf{y}\|+D} \frac{\arccos \left(\frac{r^{2}+\|\mathbf{y}\|^{2}-D^{2}}{2\|\mathbf{y}\| r}\right)}{1+\frac{r^{\alpha}}{s P}} r \mathrm{~d} r\right)\right] \\
& \stackrel{(a)}{=} \exp \left(-\pi \lambda_{2} \frac{(s P)^{2 / \alpha}}{\operatorname{sinc}(2 / \alpha)}\right) \times \exp \left[-2 \pi \lambda_{1} \int_{D}^{\infty}(1-\exp (f(v))) v \mathrm{~d} v\right],
\end{aligned}
$$

where the second term in (a) follows from the PGFL of a PPP, and then by substituting $\|\mathbf{y}\|=v$ and $f(v)=\int_{v-D}^{v+D} \arccos \left(\frac{r^{2}+v^{2}-D^{2}}{2 v r}\right) \frac{2 \lambda_{2}}{1+\frac{r \alpha}{P s}} r \mathrm{~d} r$. Since by definition of the typical node in this case, there are no points of $\Phi_{1}$ in $\mathbf{b}(0, D)$, the lower bound of integral in the above expression is $D$. 


\section{A.5 Proof of Theorem 4}

In order to derive the lower bound on the Laplace transform of interference, the interference is overestimated by considering only two holes that are closest to the typical node. The setup is illustrated in Fig. 3.3. The idea is to first derive the Laplace transform conditioned on $V_{1}=\left\|\mathbf{y}_{1}\right\|, V_{2}=\left\|\mathbf{y}_{2}\right\|$, and $\phi$, which is the angle between two holes. Deconditioning on these random variables will yield the final result. The details are as follows:

$$
\begin{aligned}
& \mathcal{L}_{I}(s) \stackrel{(\text { a) }}{\geq} \mathbb{E}\left[\exp \left(-s \sum_{\mathbf{x} \in \Phi_{2} \cap \Xi_{C}^{c}} P h_{\mathbf{x}}\|\mathbf{x}\|^{-\alpha}\right)\right] \\
& =\mathbb{E}\left[\mathbb{E}_{\Phi_{2}}\left[\prod_{\mathbf{x} \in \Phi_{2} \cap \Xi_{C}^{c}} \mathbb{E}_{h_{\mathbf{x}}}\left[\exp \left(-s P h_{\mathbf{x}}\|\mathbf{x}\|^{-\alpha}\right)\right]\right]\right. \\
& \stackrel{(\mathrm{b})}{=} \mathbb{E}\left[\mathbb{E}_{\Phi_{2}}\left[\prod_{\mathbf{x} \in \Phi_{2} \cap \Xi_{C}^{c}} \frac{\mathrm{d} \mathbf{x}}{1+s P\|\mathbf{x}\|^{-\alpha}}\right]\right] \stackrel{(\mathrm{c})}{=} \mathbb{E}\left[\exp \left(-\lambda_{2} \int_{\mathbb{R}^{2} \backslash \Xi_{C}} \frac{\mathrm{d} \mathbf{x}}{1+\frac{\|\mathbf{x}\|^{\alpha}}{s P}}\right)\right] \\
& =\mathbb{E}\left[\exp \left(-\lambda_{2}\left(\int_{\mathbb{R}^{2}} \frac{\mathrm{d} \mathbf{x}}{1+\frac{\|\mathbf{x}\|^{\alpha}}{s P}}-\int_{\Xi_{C}} \frac{\mathrm{d} \mathbf{x}}{1+\frac{\|\mathbf{x}\|^{\alpha}}{s P}}\right)\right)\right] \\
& =\mathbb{E}[\exp \left(-\pi \lambda_{2} \frac{(s P)^{2 / \alpha}}{\operatorname{sinc}(2 / \alpha)}\right) \underbrace{\exp \left(\lambda_{2} \int_{\mathcal{C}_{1}} \frac{\mathrm{d} \mathbf{x}}{1+\frac{\|\mathbf{x}\|^{\alpha}}{s P}}\right)}_{\text {closest hole }} \underbrace{\exp \left(\lambda_{2} \int_{\mathcal{C}_{2}} \frac{\mathrm{d} \mathbf{x}}{1+\frac{\|\mathbf{x}\|^{\alpha}}{s P}}\right)}_{\text {second closest hole }} \\
& \underbrace{\exp \left(-\lambda_{2} \int_{\mathcal{C}_{1} \cap \mathcal{C}_{2}} \frac{\mathrm{d} \mathbf{x}}{1+\frac{\|\mathbf{x}\|^{\alpha}}{s P}}\right)}_{\text {intersection of the two holes }}] \\
& \stackrel{(\mathrm{d})}{=} \exp \left(-\pi \lambda_{2} \frac{(s P)^{2 / \alpha}}{\operatorname{sinc}(2 / \alpha)}\right)\left(\frac{1}{2 \pi} \int_{D}^{\infty} \int_{v_{1}}^{\infty} \int_{-\pi}^{\pi} \exp \left(\int_{v_{1}-D}^{v_{1}+D_{2}} \frac{2 \pi \lambda_{c 1}(r)}{1+\frac{r^{\alpha}}{s P}} r \mathrm{~d} r\right)\right. \\
& \left.\exp \left(\int_{v_{2}-D}^{v_{2}+D_{2}} \frac{2 \pi \lambda_{c 2}(r)}{1+\frac{r^{\alpha}}{s P}} r \mathrm{~d} r\right) \exp (-\lambda_{2} \underbrace{\int_{\mathcal{C}_{1} \cap \mathcal{C}_{2}} \frac{\mathrm{d} \mathbf{x}}{1+\frac{\|\mathbf{x}\|^{\alpha}}{s P}}}_{\mathcal{B}\left(v_{1}, v_{2}, \phi\right)}) f_{V_{1} V_{2}}\left(v_{1}, v_{2}\right) \mathrm{d} \phi \mathrm{d} v_{2} \mathrm{~d} v_{1}\right),
\end{aligned}
$$


where $\Xi_{C}$ in step (a) is $\Xi_{C}=\mathcal{C}_{1} \cup \mathcal{C}_{2}$ with $\mathcal{C}_{1}=\mathbf{b}\left(\mathbf{y}_{1}, D\right)$ and $\mathcal{C}_{2}=\mathbf{b}\left(\mathbf{y}_{2}, D\right)$, and $\lambda_{c 1}(r)$ and $\lambda_{c 2}(r)$ in the last step are $\lambda_{c 1}(r)=\frac{\lambda_{2}}{\pi} \arccos \left(\frac{r^{2}+v_{1}^{2}-D^{2}}{2 v_{1} r}\right)$ and $\lambda_{c 2}(r)=\frac{\lambda_{2}}{\pi} \arccos \left(\frac{r^{2}+v_{2}^{2}-D^{2}}{2 v_{2} r}\right)$. Step (b) follows from $h_{\mathbf{x}} \sim \exp (1)$, (c) from the PGFL of PPP, and (d) by deconditioning on the distributions of $V_{1}, V_{2}$, and $\phi$. Note that while the joint distribution of $V_{1}$ and $V_{2}$ is given by (3.14), $\phi$ is independent of all other random variables and is uniformly distributed between $-\pi$ and $\pi$. In step (d), the terms corresponding to the closest and second closest holes are derived on the same lines as Lemma 5 (conditioned on $V_{1}$ and $V_{2}$ ). The rest of the proof will focus on evaluating the integral $\mathcal{B}\left(v_{1}, v_{2}, \phi\right)$ that appears in the term corresponding to the intersection of the two holes. Our first goal is to find the coordinates of the points at which the two circles $\mathcal{C}_{1}$ and $\mathcal{C}_{2}$ intersect. Without loss of generality, we assume that the centers of the two circles are locates at $\mathbf{y}_{1}=\left(y_{1 \mathrm{x}}, 0\right)$ and $\mathbf{y}_{2}=$ $\left(y_{2 \mathrm{x}}, y_{2 \mathrm{y}}\right)$ while they are separated by distance $w=\sqrt{v_{1}^{2}+v_{2}^{2}-2 v_{1} v_{2} \cos \phi}$. Using the equations of the two circles, $\left(x_{\mathrm{x}}-y_{1 \mathrm{x}}\right)^{2}+x_{\mathrm{y}}{ }^{2}=D^{2}$, and $\left(x_{\mathrm{x}}-\right.$ $\left.y_{2 \mathrm{x}}\right)^{2}+\left(x_{\mathrm{y}}-y_{2 \mathrm{y}}\right)^{2}=D^{2}$, we obtain

$$
-2 x_{\mathrm{x}} y_{1 \mathrm{x}}+y_{1 \mathrm{x}}^{2}=-2 x_{\mathrm{x}} y_{2 \mathrm{x}}+y_{2 \mathrm{x}}^{2}-2 x_{\mathrm{y}} y_{2 \mathrm{y}}+y_{2 \mathrm{y}}^{2},
$$

using which we find $x_{\mathrm{y}}$ in terms of other variables. Then, substituting $x_{\mathrm{y}}$ in one of the equations of the circle, we get a quadratic equation for $x_{\mathrm{x}}$. Solving these equations, the coordinates of the intersection points can be found to 
be [87]

$$
\left(\hat{u}_{1}, \hat{t}_{1}\right)=\left(\frac{1}{2}\left(y_{1 \mathrm{x}}+y_{2 \mathrm{x}}\right)+\frac{1}{2} \sqrt{\frac{4 D^{2}}{w^{2}}-1} y_{2 \mathrm{y}}, \frac{y_{2 \mathrm{y}}}{2}+\frac{1}{2} \sqrt{\frac{4 D^{2}}{w^{2}}-1}\left(y_{1 \mathrm{x}}-y_{2 \mathrm{x}}\right)\right)
$$

$\left(\hat{u}_{2}, \hat{t}_{2}\right)=\left(\frac{1}{2}\left(y_{1 \mathrm{x}}+y_{2 \mathrm{x}}\right)-\frac{1}{2} \sqrt{\frac{4 D^{2}}{w^{2}}-1} y_{2 \mathrm{y}}, \frac{y_{2 \mathrm{y}}}{2}-\frac{1}{2} \sqrt{\frac{4 D^{2}}{w^{2}}-1}\left(y_{1 \mathrm{x}}-y_{2 \mathrm{x}}\right)\right)$

Substituting $\left(y_{1 \mathrm{x}}, y_{1 \mathrm{y}}\right)=\left(v_{1}, 0\right),\left(y_{2 \mathrm{x}}, y_{2 \mathrm{y}}\right)=\left(v_{2} \cos \phi, v_{2} \sin \phi\right)$ in these expressions, we get

$$
\begin{aligned}
& \left(\hat{u}_{1}, \hat{t}_{1}\right) \\
& =\frac{1}{2}\left(v_{1}+v_{2} \cos \phi+\sqrt{\frac{4 D^{2}}{w^{2}}-1} v_{2} \sin \phi, v_{2} \sin \phi+\sqrt{\frac{4 D^{2}}{w^{2}}-1}\left(v_{1}-v_{2} \cos \phi\right)\right)
\end{aligned}
$$

and

$$
\begin{aligned}
& \left(\hat{u}_{2}, \hat{t}_{2}\right) \\
& =\frac{1}{2}\left(v_{1}+v_{2} \cos \phi-\sqrt{\frac{4 D^{2}}{w^{2}}-1} v_{2} \sin \phi, v_{2} \sin \phi-\sqrt{\frac{4 D^{2}}{w^{2}}-1}\left(v_{1}-v_{2} \cos \phi\right)\right) .
\end{aligned}
$$


Note that the overlap between the circles will happen only when the distance between their centers is smaller than $2 D$, i.e., $w \leq 2 D$. For a given $v_{1}$, and $v_{2} \geq v_{1}$, it can be easily deduced that the overlap occurs only when $\phi \leq \hat{\phi}$, where $\hat{\phi}=\arccos \left(\frac{v_{1}^{2}+v_{2}^{2}-4 D^{2}}{2 v_{1} v_{2}}\right)$. The integral in the term corresponding to the intersection of the two circles, $\mathcal{B}\left(v_{1}, v_{2}, \phi\right)$, can now we derived as

$$
\begin{aligned}
& \mathcal{B}\left(v_{1}, v_{2}, \phi\right)=\int_{\mathcal{C}_{1} \cap \mathcal{C}_{2}} \frac{\mathrm{d} \mathbf{x}}{1+\frac{\|\mathbf{x}\|^{\alpha}}{s P}} \\
& =\left\{\begin{array}{cc}
\int_{\hat{u}_{2}}^{\hat{u}_{1}} \int_{y_{2 \mathrm{y}}-\sqrt{D^{2}-\left(x_{\mathrm{x}}-y_{2 \mathrm{x}}\right)^{2}}}^{\sqrt{D^{2}-\left(x_{\mathrm{x}}-y_{1 \mathrm{x}}\right)^{2}}} \frac{\mathrm{d} x_{\mathrm{y}} \mathrm{d} x_{\mathrm{x}}}{1+\frac{\left(x_{\mathrm{x}}^{2}+x_{\mathrm{y}}^{2}\right)^{\frac{\alpha}{2}}}{s P}} & 0 \leq \phi<\hat{\phi} \\
0 & |\phi| \geq \hat{\phi} \quad, \\
\int_{\hat{u}_{1}}^{\hat{u}_{2}} \int_{-\sqrt{D^{2}-\left(x_{\mathrm{x}}-y_{1 \mathrm{x}}\right)^{2}}}^{y_{2 \mathrm{y}}+\sqrt{D^{2}-\left(x_{\mathrm{x}}-y_{2 \mathrm{x}}\right)^{2}}} \frac{\mathrm{d} x_{\mathrm{y}} \mathrm{d} x_{\mathrm{x}}}{1+\frac{\left(x_{\mathrm{x}}^{2}+x_{\mathrm{y}}^{2}\right)^{\frac{\alpha}{2}}}{s P}} & -\hat{\phi}<\phi<0
\end{array}\right.
\end{aligned}
$$

where $\|\mathbf{x}\|=\sqrt{x_{\mathrm{x}}^{2}+x_{\mathrm{y}}^{2}}, \hat{\phi}=\arccos \left(\frac{v_{1}^{2}+v_{2}^{2}-4 D^{2}}{2 v_{1} v_{2}}\right)$. This completes the proof.

\section{A.6 Proof of Theorem 5}

We consider $k$ closest holes to the typical node of the PHP. The setup is illustrated in Fig. 3.4. Denoting the locations of the holes by $\mathbf{y}_{1}, \ldots, \mathbf{y}_{i}, \ldots, \mathbf{y}_{k}$, the interference field in this case is modeled by $\Omega=\Phi_{2} \cap\left\{\mathcal{C}_{1} \cup\left\{\cup_{i=2}^{k} \mathbf{d}\left(\mathbf{y}_{i}, D\right)\right\}\right\}^{c}$, where $\Omega \supset \Psi$ and $\mathbf{d}\left(\mathbf{y}_{i}, D\right)$ is defined in (3.16). This approach overestimates the interference power and hence leads to a lower bound on the Laplace transform of interference. Let $I=\sum_{\mathbf{x} \in \Omega} P h_{\mathbf{x}}\|\mathbf{x}\|^{-\alpha}$, the Laplace transform of 
interference conditioned on $V_{1}=\left\|\mathbf{y}_{1}\right\|, V_{2}=\left\|\mathbf{y}_{2}\right\|, \ldots, V_{k}=\left\|\mathbf{y}_{k}\right\|$ is

$$
\begin{aligned}
& \mathcal{L}_{I \mid\left\|\mathbf{y}_{1}\right\|, \ldots,\left\|\mathbf{y}_{k}\right\|}(s)=\mathbb{E}\left[\exp \left(-s \sum_{\mathbf{x} \in \Omega} P h_{\mathbf{x}}\|\mathbf{x}\|^{-\alpha}\right)\right] \stackrel{(\mathrm{a})}{=} \mathbb{E}_{\Omega}\left[\prod_{\mathbf{x} \in \Omega} \frac{1}{1+s P\|\mathbf{x}\|^{-\alpha}}\right] \\
& \stackrel{(\text { b) }}{=} \exp \left(-\lambda_{2}\left(\int_{\mathbb{R}^{2}} \frac{1}{1+\frac{\|\mathbf{x}\|^{\alpha}}{s P}} \mathrm{~d} \mathbf{x}-\int_{\mathbf{b}\left(\mathbf{y}_{1}, D\right)} \frac{1}{1+\frac{\|\mathbf{x}\|^{\alpha}}{s P}} \mathrm{~d} \mathbf{x}-\sum_{i=2}^{k} \int_{\mathbf{d}\left(\mathbf{y}_{i}, D\right)} \frac{1}{1+\frac{\|\mathbf{x}\|^{\alpha}}{s P}} \mathrm{~d} \mathbf{x}\right)\right) \\
& \stackrel{(\mathrm{c})}{=} \exp \left(-\pi \lambda_{2} \frac{(s P)^{2 / \alpha}}{\operatorname{sinc}(2 / \alpha)}\right) \times \exp \left(2 \lambda_{2} \int_{\left\|\mathbf{y}_{1}\right\|-D}^{\left\|\mathbf{y}_{1}\right\|+D} \frac{\arccos \left(\frac{r^{2}+\left\|\mathbf{y}_{1}\right\|^{2}-D^{2}}{2\left\|\mathbf{y}_{1}\right\| r}\right)}{1+\frac{r^{\alpha}}{s P}} r \mathrm{~d} r\right) \\
& \times \prod_{i=2}^{k} \exp \left(2 \lambda_{2} \int_{\max \left(\left\|\mathbf{y}_{i}\right\|-D,\left\|\mathbf{y}_{i-1}\right\|+D\right)}^{\left\|\mathbf{y}_{i}\right\|+D} \frac{\arccos \left(\frac{r^{2}+\left\|\mathbf{y}_{i}\right\|^{2}-D^{2}}{2\left\|\mathbf{y}_{i}\right\| r}\right)}{1+\frac{r^{\alpha}}{s P}} r \mathrm{~d} r\right)
\end{aligned}
$$

where (a) follows from $h_{\mathbf{x}} \sim \exp (1)$, (b) from PGFL of PPP along with the fact that the points of the baseline $\mathrm{PPP} \Phi_{2}$ that are located in the closest hole $\mathbf{b}\left(\mathbf{y}_{1}, D\right)$ and the sets $\cup_{i=2}^{k} \mathbf{d}\left(\mathbf{y}_{i}, D\right)$ should be removed, and (c) from the cosine-law by using the same argument applied in the proof of Lemma 5 . Finally, deconditioning the resulting expression with respect to the distances of the centers of the $k$ holes from the typical node, $V_{1}, V_{2}, \ldots, V_{k}$, with joint distribution given by

$$
\begin{aligned}
& f_{V_{1} V_{2} . . V_{k}}\left(v_{1}, v_{2}, . ., v_{k}\right) \\
& \quad=\left(2 \pi \lambda_{1}\right)^{k} v_{1} v_{2} \ldots v_{k} \exp \left(-\pi \lambda_{1}\left(v_{k}^{2}-D^{2}\right)\right), \quad D<v_{1}<v_{2}<\ldots<v_{k},
\end{aligned}
$$

completes the proof. Here, the joint PDF $f_{V_{1} V_{2} . . V_{k}}($.$) is derived by using the$ same argument as in equation (3.14) [78]. 


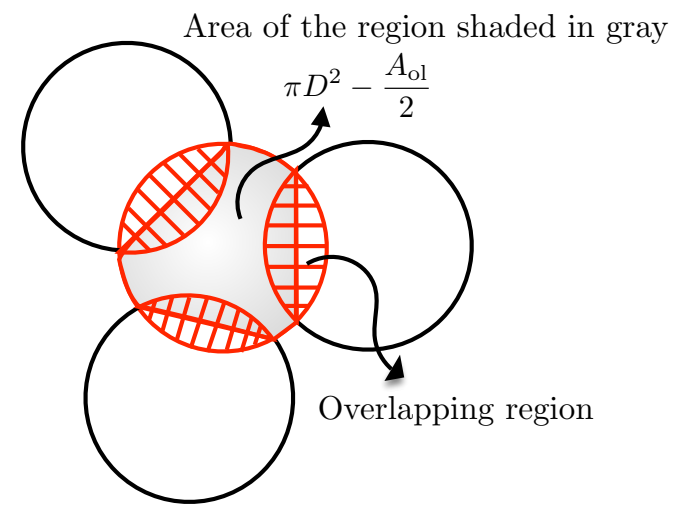

Figure A.1: Illustration of the overlap effect for a typical hole in the PHP. The area of the region shaded in gray color is $\pi D^{2}-\frac{A_{\mathrm{ol}}}{2}$ and in red stripes is $A_{\mathrm{ol}}$.

\section{A.7 Proof of Proposition 1}

Consider the illustration of overlapping circles shown in Fig. A.1. While defining the PHP, points of the baseline PPP $\Phi_{2}$ lying inside these circles are removed. In other words, the "union" of such overlapping circles is removed. However, in our upper bound given by Theorem 3, we removed each such circle separately of the others leading to the removal of the overlapping parts multiple times. Therefore, while the average number of points removed per unit area of these circles was $\lambda_{2}$ in the PHP, the average number of points removed per unit area of such circles in Theorem 3, denoted by $\lambda_{\text {eff }}$, is $\lambda_{\text {eff }}>$ $\lambda_{2}$. To maintain tractability later in the proof, we confine to the pairwise overlaps, meaning the total overlap area for a circle, denoted by $A_{\mathrm{ol}}$, is the sum of pairwise overlap areas of this circle with the other circles. Now to estimate $\lambda_{\text {eff }}$, consider the shaded region (in gray color) in Fig. A.1 with area $\pi D^{2}-\frac{A_{\mathrm{ol}}}{2}$, which represents the effective contribution of each hole in the PHP. 
Due to overlaps, the total average number of points removed under Theorem 3 from this region is $\lambda_{2} \pi D^{2}$. Using this fact, $\lambda_{\text {eff }}$ can be estimated as

$$
\lambda_{\mathrm{eff}} \times\left(\pi D^{2}-\frac{A_{\mathrm{ol}}}{2}\right)=\lambda_{2} \pi D^{2} \Rightarrow \lambda_{\mathrm{eff}}=\frac{\lambda_{2}}{\left(1-\frac{A_{\mathrm{ol}}}{2 \pi D^{2}}\right)} .
$$

The above expression shows that $\frac{1}{\left(1-\frac{A_{\text {ol }}}{2 \pi D^{2}}\right)}$ times more points are removed in the upper bound given by Theorem 3. To compensate for this effect, we first proceed as in Theorem 3 and then rescale the second term (that captures the effect of removing holes) as follows:

$$
\begin{aligned}
& \mathcal{L}_{I}(s) \stackrel{(\mathrm{a})}{\simeq} \\
& \mathbb{E}_{\Phi_{1}}\left[\exp \left(-\lambda_{2}\left(\int_{\mathbb{R}^{2}} \frac{\mathrm{d} \mathbf{x}}{1+\frac{\|\mathbf{x}\|^{\alpha}}{s P}}-\left(1-\frac{A_{\mathrm{ol}}}{2 \pi D^{2}}\right) \sum_{\mathbf{y} \in \Phi_{1}} \int_{\mathbf{b}(\mathbf{y}, D)} \frac{\mathrm{d} \mathbf{x}}{1+\frac{\|\mathbf{x}\|^{\alpha}}{s P}}\right)\right)\right] \\
& =\exp \left(-\pi \lambda_{2} \frac{(s P)^{2 / \alpha}}{\operatorname{sinc}(2 / \alpha)}\right) \times \\
& \mathbb{E}_{\Phi_{1}}\left[\prod_{\mathbf{y} \in \Phi_{1}} \exp \left(2 \lambda_{2}\left(1-\frac{A_{\mathrm{ol}}}{2 \pi D^{2}}\right) \int_{\|\mathbf{y}\|-D}^{\|\mathbf{y}\|+D} \frac{\arccos \left(\frac{r^{2}+\|\mathbf{y}\|^{2}-D^{2}}{2\|\mathbf{y}\| r}\right)}{1+\frac{r^{\alpha}}{s P}} r \mathrm{~d} r\right)\right] \\
& \stackrel{(\mathrm{b})}{=} \exp \left(-\pi \lambda_{2} \frac{(s P)^{2 / \alpha}}{\operatorname{sinc}(2 / \alpha)}\right) \\
& \times \exp \left[-2 \pi \lambda_{1} \int_{D}^{\infty}\left(1-\exp \left(f(v)\left(1-\frac{A_{\mathrm{ol}}}{2 \pi D^{2}}\right)\right)\right) v \mathrm{~d} v\right]
\end{aligned}
$$

where (a) follows from $h_{\mathbf{x}} \sim \exp (1)$ and the PGFL of a PPP. The second term is rescaled as discussed above. Further, the second term in (b) follows from the PGFL of a PPP, and then by substituting $\|\mathbf{y}\|=v$ and $f(v)=$ $\int_{v-D}^{v+D} \arccos \left(\frac{r^{2}+v^{2}-D^{2}}{2 v r}\right) \frac{2 \lambda_{2}}{1+\frac{r^{\alpha}}{P s}} r \mathrm{~d} r$. 
Now to determine the total average pairwise overlapping area $A_{\mathrm{ol}}$, consider a circle of interest $\mathbf{b}(\mathbf{y}, D)$. Note that only the circles with centers located inside the region $\mathbf{b}(\mathbf{y}, 2 D)$ will overlap with this circle. Denote the number of circles in this region (besides the circle of interest) by $K$, where $K$ has Poisson distribution with mean $\lambda_{1} 4 \pi D^{2}$. For one of these circles, say $\mathbf{b}\left(\mathbf{y}^{\prime}, D\right)$, the area of overlapping region with the circle of interest is

$$
A(\hat{z})=2 D^{2} \arccos \left(\frac{\hat{z}}{2 D}\right)-\hat{z} D \sqrt{1-\left(\frac{\hat{z}}{2 D}\right)^{2}}
$$

where $\hat{z}=\left\|\mathbf{y}-\mathbf{y}^{\prime}\right\|$. Now conditioned on $K=k$, the $k$ circles are independent and uniformly distributed over $\mathbf{b}(\mathbf{y}, 2 D)$. Hence, the PDF of distance $\hat{z}$ is $f_{\hat{Z}}(\hat{z})=\frac{2 \hat{z}}{4 D^{2}}$. Then the average area of overlapping region between the circle of interest and another circle is

$$
\begin{aligned}
\bar{A} & =\mathbb{E}_{\hat{Z}}[A(\hat{z})] \\
& =\left(\int_{0}^{2 D}\left(2 D^{2} \arccos \left(\frac{\hat{z}}{2 D}\right)-\hat{z} D \sqrt{1-\left(\frac{\hat{z}}{2 D}\right)^{2}}\right) f_{\hat{Z}}(\hat{z}) \mathrm{d} \hat{z}\right)=\frac{\pi D^{2}}{4} .
\end{aligned}
$$

Now the pairwise overlap area for the circle of interest with $k$ other circles is $k \bar{A}$. Since there are on average $\lambda_{1} 4 \pi D^{2}$ circles that overlap with the circle of interest, the average pairwise overlap area is $\lambda_{1} 4 \pi D^{2} \bar{A}=\lambda_{1} \pi^{2} D^{4}$. Since the maximum overlap is bounded above by $\pi D^{2}$, we get $A_{\mathrm{ol}}=\min \left(\lambda_{1} \pi^{2} D^{4}, \pi D^{2}\right)$. This completes the proof. 


\section{Appendix B}

\section{B.1 Proof of Lemma 7}

We consider only the closest hole to the typical user, thus leading to a lower bound on the serving distance $Z_{2}$ which is denoted by $\hat{Z}_{2}$. Conditioned on the distance $Z_{1}$, the distribution of $\hat{Z}_{2}$ is

$$
\begin{aligned}
& \mathbb{P}\left(\hat{Z}_{2}>\hat{z_{2}} \mid Z_{1}\right) \\
& =\mathbb{P}\left(\text { Number of points of } \Phi_{2} \text { in the set }\left\{\mathbf{b}\left(0, \hat{z_{2}}\right)\right\}=0\right) \\
& =\exp \left(-\lambda_{2}\left(\pi{\hat{z_{2}}}^{2}-A_{\text {ins }}\left(\hat{z_{2}}, Z_{1}\right)\right)\right), z_{1}>0 .
\end{aligned}
$$

where $\hat{z_{2}}$ and $Z_{1}$ denote the distance from the typical user to its serving small cell and its distance to the closest macro BS. Further, $A_{\text {ins }}\left(\hat{z_{2}}, Z_{1}\right)$ denotes the area of intersection between two circles $\mathbf{b}\left(0, \hat{z_{2}}\right)$ and $\mathbf{b}\left(Z_{1}, D\right)$. The result now follows by differentiating the above expression. 


\section{B.2 Proof of Theorem 6}

Coverage probability conditioned on the serving distance when $Z_{1} \geq D$ is

$$
\begin{aligned}
\mathrm{P}_{\mathrm{c} 1 \mid Z_{1} \geq D} \geq & \mathbb{E}\left[\exp \left(-s\left(\sum_{\mathbf{x}_{2} \in \Omega \cap \mathbf{b}^{c}\left(\mathbf{x}_{1}^{*}, D\right)} P_{2} h_{\mathbf{x}_{2}}\left\|\mathbf{x}_{2}\right\|^{-\alpha}+\sum_{\mathbf{x}_{1} \in \Phi_{1} / \mathbf{x}_{1}^{*}} P_{1} h_{\mathbf{x}_{1}}\left\|\mathbf{x}_{1}\right\|^{-\alpha}\right)\right)\right] \\
& \stackrel{(a)}{=} \mathbb{E}_{\Phi_{1} \mid Z_{1}}\left[\exp \left(-\lambda_{2}\left(\int_{\mathbb{R}^{2}} \frac{\mathrm{d} \mathbf{x}_{2}}{1+\frac{\left\|\mathbf{x}_{2}\right\|^{\alpha}}{s P_{2}}}-\int_{\mathbf{b}\left(\mathbf{x}_{1}^{*}, D\right)} \frac{\mathrm{d} \mathbf{x}_{2}}{1+\frac{\left\|\mathbf{x}_{2}\right\|^{\alpha}}{s P_{2}}}\right)\right)\right. \\
& \left.\times \prod_{\mathbf{x}_{1} \in \Phi_{1} / \mathbf{x}_{1}^{*}} \zeta\left(s,\left\|\mathbf{x}_{1}\right\|\right)\right] \stackrel{(\mathrm{b})}{=} \hat{\mathcal{G}}_{1}^{(1)}(s) \times \exp \left(f\left(s, z_{1}\right)\right) \times \\
& \exp \left(-2 \pi \lambda_{1} \int_{z_{1}}^{\infty}(1-\zeta(s, v)) v \mathrm{~d} v\right)
\end{aligned}
$$

where $\zeta(s, v)=\frac{1}{1+s P_{1} v^{-\alpha}}$. Step (a) follows from taking expectations over channel gains $h_{\mathbf{x}_{k}} \sim \exp (1)$ and by using the PGFL of the PPP [71], $\hat{\mathcal{G}}^{(1)}(s)=$ $\exp \left(-\pi \lambda_{2} \frac{(s P)^{2 / \alpha}}{\operatorname{sinc}(2 / \alpha)}\right)$, as shown in [73, Appendix B] and the second term in (b) follows from conditioning on the $Z_{1}$ using the cosine-law (as shown in Fig. 4.1): $r^{2}+\left\|\mathbf{x}_{1}^{*}\right\|^{2}-2 r\left\|\mathbf{x}_{1}^{*}\right\| \cos \theta(r)=D^{2}$ along with converting form Cartesian to polar coordinates by substituting $f\left(s, z_{1}\right)=\int_{\left|z_{1}-D\right|}^{z_{1}+D} \arccos \left(\frac{r^{2}+z_{1}^{2}-D^{2}}{2 z_{1} r}\right) \frac{2 \lambda_{2}}{1+\frac{r^{\alpha}}{P_{2} s}} r \mathrm{~d} r$ which is the effective interference power "removed" by the closest hole. It should be noted that the proof of $\mathrm{P}_{\mathrm{c} 1 \mid Z_{1}<D}$ is similar to the proof of $\mathrm{P}_{\mathrm{c} 1 \mid Z_{1} \geq D}$ except that the closest point of $\Phi_{2}$ is at least a distance $D-z_{1}$ away from the typical point located inside the hole, and hence leads to $\mathcal{G}_{1}^{(1)}(s)=\exp \left(\int_{D-z_{1}}^{\infty} \frac{-2 \pi \lambda_{2} r \mathrm{~d} r}{1+\frac{r^{\alpha}}{s P_{2}}}\right)$.

The final result follows from deconditioning over serving distance $Z_{1}$ whose distribution is given by (4.2). 


\section{B.3 Proof of Theorem 7}

The coverage probability in this case conditioned on the serving distance when $Z_{1} \geq D$ is

$$
\begin{aligned}
\mathrm{P}_{\mathrm{c} 1 \mid Z_{1} \geq D} & =\mathbb{E}\left[\exp \left(-s\left(\sum_{\mathbf{x}_{2} \in \Phi_{2}} P_{2} h_{\mathbf{x}_{2}}\left\|\mathbf{x}_{2}\right\|^{-\alpha}+\sum_{\mathbf{x}_{1} \in \Phi_{1} / \mathbf{x}_{1}^{*}} P_{1} h_{\mathbf{x}_{1}}\left\|\mathbf{x}_{1}\right\|^{-\alpha}\right)\right)\right] \\
& \stackrel{(\mathrm{a})}{=} \mathbb{E}_{\Phi_{1} \mid Z_{1}}\left[\exp \left(-\lambda_{2}\left(\int_{\mathbb{R}^{2}} \frac{\mathrm{d} \mathbf{x}_{2}}{1+\frac{\left\|\mathbf{x}_{2}\right\|^{\alpha}}{s P_{2}}}-\int_{\Xi_{D}} \frac{\mathrm{d} \mathbf{x}_{2}}{1+\frac{\left\|\mathbf{x}_{2}\right\|^{\alpha}}{s P_{2}}}\right)\right)\right. \\
& \left.\times \prod_{\mathbf{x}_{1} \in \Phi_{1} / \mathbf{x}_{1}^{*}} \zeta\left(s,\left\|\mathbf{x}_{1}\right\|\right)\right] \stackrel{(\mathrm{b})}{\leq} \hat{\mathcal{G}}_{1}^{(1)}(s) \times \mathbb{E}_{\Phi_{1} \mid Z_{1}}[ \\
& \exp \left(\lambda_{2}\left(\sum_{\mathbf{x}_{1} \in \Phi_{1}} \int_{\mathbf{b}\left(\mathbf{x}_{1}, D\right)} \frac{\mathrm{d} \mathbf{x}_{2}}{1+\frac{\left\|\mathbf{x}_{2}\right\|^{\alpha}}{s P_{2}}}\right) \prod_{\mathbf{x}_{1} \in \Phi_{1} / \mathbf{x}_{1}^{*}} \zeta\left(s,\left\|\mathbf{x}_{1}\right\|\right)\right] \\
& \stackrel{(\mathrm{c})}{=} \mathcal{G}_{1}^{(1)}(s) \exp \left(f\left(s,\left\|\mathbf{x}_{1}^{*}\right\|\right)\right) \mathbb{E}_{\Phi_{1} \mid Z_{1}} \prod_{\mathbf{x}_{1} \in \Phi_{1} / \mathbf{x}_{1}^{*}} \exp \left(f\left(s,\left\|\mathbf{x}_{1}\right\|\right)\right) \\
& \left.\zeta\left(s,\left\|\mathbf{x}_{1}\right\|\right)\right] \stackrel{(\mathrm{d})}{=} \mathcal{G}_{1}^{(1)}(s) \times \exp \left(f\left(s, z_{1}\right)\right) \times \\
& \exp \left(-2 \pi \lambda_{1} \int_{z_{1}}^{\infty}(1-\exp (f(s, v)) \times \zeta(s, v)) v \mathrm{~d} v\right)
\end{aligned}
$$

where $\Phi_{2}=\Omega \backslash \Xi_{D}, \Xi_{D}$ in step (a) is $\triangleq \bigcup_{\mathbf{x}_{1} \in \Phi_{1}} \mathbf{b}\left(\mathbf{x}_{1}, D\right)$ as defined in (4.1), $\zeta(s, u)=\frac{1}{1+s P_{1} u^{-\alpha}}$, (a) comes from taking expectations over channel gains $h_{\mathbf{x}_{k}} \sim \exp (1)$ and by using the PGFL of the PPP $\Omega$ given $\Xi_{D}, \hat{\mathcal{G}}^{(1)}(s)=$ $\exp \left(-\pi \lambda_{2} \frac{\left(s P_{2}\right)^{2 / \alpha}}{\operatorname{sinc}(2 / \alpha)}\right)$, (b) is obtained by ignoring the effect of overlaps and the fact that each hole is carved out individually from the baseline PPP $\Omega$ which leads to an upper bound for the conditional coverage probability of the typical user which is served by macro BS. In the second term of (c), 
$f(s, u)=\int_{|u-D|}^{u+D} \arccos \left(\frac{r^{2}+u^{2}-D^{2}}{2 u r}\right) \frac{2 \lambda_{2}}{1+\frac{r^{\alpha}}{P_{2} s}} r \mathrm{~d} r$. Finally, the last term in (d) follows from the PGFL of a PPP [71] along with converting form Cartesian to polar coordinates by substituting $v=\left\|\mathbf{x}_{1}\right\|$ and $f(s, v)$. The proof for the $\mathrm{P}_{\mathrm{c} 1 \mid Z_{1}<D}$ follows on the same line as the proof of $\mathrm{P}_{\mathrm{c} 1 \mid Z_{1} \geq D}$ except that $\mathcal{G}_{1}^{(1)}(s)=\exp \left(\int_{D-z_{1}}^{\infty} \frac{-2 \pi \lambda_{2} r \mathrm{~d} r}{1+\frac{r^{\alpha}}{s P_{2}}}\right)$ because the closest point of $\Phi_{2}$ is at least a distance $D-z_{1}$ away from the typical user within the hole. The final result follows from deconditioning over serving distance $Z_{1}$.

\section{B.4 Proof of Theorem 8}

We consider only the closest hole in the interference field. The coverage probability of a typical user which connects to the nearest small cell conditioned on the fixed distances $Z_{1}$ and $\hat{Z}_{2}$, is lower bounded by

$$
\begin{aligned}
\mathrm{P}_{\mathrm{c}_{2} \mid \hat{z}_{2}, Z_{1}}(s) \geq & \mathbb{E}\left[\exp \left(-s\left(\sum_{\substack{\mathbf{x}_{2} \in \Omega \cap \mathbf{b}^{c}\left(\mathbf{x}_{1}^{*}, D\right),\left\|\mathbf{x}_{2}\right\|>z_{2}}} P_{2} h_{\mathbf{x}_{2}}\left\|\mathbf{x}_{2}\right\|^{-\alpha}+\sum_{\mathbf{x}_{1} \in \Phi_{1}} P_{1} h_{\mathbf{x}_{1}}\left\|\mathbf{x}_{1}\right\|^{-\alpha}\right)\right)\right] \\
& \stackrel{(\mathrm{a})}{=} \mathcal{G}_{1}^{(2)}\left(s, \hat{z_{2}}\right) \exp \left(\lambda_{2} \int_{\substack{\mathbf{b}\left(\mathbf{x}_{1}^{*}, D\right),\left\|\mathbf{x}_{2}\right\|>\hat{z}_{2}}} \frac{\mathrm{d} \mathbf{x}_{2}}{1+\frac{\left\|\mathbf{x}_{2}\right\| \alpha}{s P_{2}}}\right) \mathbb{E}_{\Phi_{1} \mid Z_{1}}\left[\prod_{\mathbf{x}_{1} \in \Phi_{1}} \zeta\left(s,\left\|\mathbf{x}_{1}\right\|\right)\right] \\
& \stackrel{(\mathrm{b})}{=} \mathcal{G}_{1}^{(2)}\left(s, \hat{z_{2}}\right) \exp \left(g\left(s, z_{1}, \hat{z_{2}}\right)\right) \zeta\left(s, z_{1}\right) \\
& \times \exp \left(-2 \pi \lambda_{1} \int_{z_{1}}^{\infty}(1-\zeta(s, v)) v \mathrm{~d} v\right)
\end{aligned}
$$

where in step $(\mathrm{a}), \mathcal{G}_{1}^{(2)}\left(s, \hat{z_{2}}\right)=\exp \left(\int_{\hat{z_{2}}}^{\infty} \frac{-2 \pi \lambda_{2} r \mathrm{~d} \mathbf{r}}{1+\frac{r \alpha}{s P_{2}}}\right), \zeta(s, u)=\frac{1}{1+s P_{1} u^{-\alpha}}$, (a) follows from the fact that the field of possible interferers involves all of the 
small cell BSs outside the closest hole (except the serving BS), the second term of (b) can be shown by the expression

$$
g\left(s, u, \hat{z_{2}}\right)=\exp \left(\int_{\max \left(\hat{z_{2}},|u-D|\right)}^{\max \left(\hat{z_{2}}, u+D\right)} \arccos \left(\frac{r^{2}+u^{2}-D^{2}}{2 u r}\right) \frac{2 \lambda_{2}}{1+\frac{r^{\alpha}}{s P_{2}}} r \mathrm{~d} r\right)
$$

which is due to the fact that the field of effective interferers is out of $\mathbf{b}\left(0, \hat{z_{2}}\right)$ that appears in the lower and upper bounds of integral expression which are $\max \left(\hat{z_{2}},||\left|\mathbf{x}_{1} \|-D\right|\right)$ and $\max \left(\hat{z_{2}},\left\|\mathbf{x}_{1}\right\|+D\right)$, and the term of $\zeta\left(s, z_{1}\right)$ denotes the effective interference from the closest macro BS at the typical point due to conditioning on $Z_{1}=\left\|\mathbf{x}_{1}^{*}\right\|$. The final result follows from deconditioning over serving distance $\hat{Z}_{2}$ and then over distance $Z_{1}$. 


\section{B.5 Proof of Theorem 9}

The coverage probability of a typical user when it is served by its closest small cell (conditioned on the fixed distances $Z_{1}$ and $\hat{Z}_{2}$ is upper bounded by

$$
\begin{aligned}
\mathrm{P}_{\mathrm{c}_{2} \mid \hat{z}_{2}, Z_{1}}(s)= & \mathbb{E}\left[\exp \left(-s\left(\sum_{\mathbf{x}_{2} \in \Phi_{2},\left\|\mathbf{x}_{2}\right\|>\hat{z_{2}}} P_{2} h_{\mathbf{x}_{2}}\left\|\mathbf{x}_{2}\right\|^{-\alpha}+\sum_{\mathbf{x}_{1} \in \Phi_{1}} P_{1} h_{\mathbf{x}_{1}}\left\|\mathbf{x}_{1}\right\|^{-\alpha}\right)\right)\right] \\
\leq & \mathcal{G}_{1}^{(2)}\left(s, \hat{z_{2}}\right) \mathbb{E}_{\Phi_{1} \mid Z_{1}}\left[\exp \left(\lambda_{2}\left(\sum_{\mathbf{x}_{1} \in \Phi_{1}} \int_{\substack{\mathbf{b}\left(\mathbf{x}_{1}, D\right),\left\|\mathbf{x}_{2}\right\|>z_{2}}} \frac{\mathrm{d} \mathbf{x}_{2}}{1+\frac{\left\|\mathbf{x}_{2}\right\| \alpha}{s P_{2}}}\right)\right)\right. \\
& \left.\prod_{\mathbf{x}_{1} \in \Phi_{1}} \frac{1}{1+s P_{1}\left\|\mathbf{x}_{1}\right\|^{-\alpha}}\right]=\mathcal{G}_{1}^{(2)}\left(s, \hat{z_{2}}\right) \times \mathbb{E}_{\Phi_{1} \mid Z_{1}}[ \\
& \prod_{\mathbf{x}_{1} \in \Phi_{1}} \exp \left(2 \lambda_{2} \int_{\max \left(\hat{z_{2}},\left\|\mathbf{x}_{1}\right\|-D \mid\right)}^{\max \left(\hat{z_{2}},\left\|\mathbf{x}_{1}\right\|+D\right) \arccos \left(\frac{r^{2}+\left\|\mathbf{x}_{1}\right\|^{2}-D^{2}}{2\left\|\mathbf{x}_{1}\right\| r}\right)}\right. \\
& \times \frac{1+\frac{r^{\alpha}}{s P_{2}}}{1+s P_{1}\left\|\mathbf{x}_{1}\right\|^{-\alpha}}=\mathcal{G}_{1}^{(2)}\left(s, \hat{z_{2}}\right) \times \exp \left(g\left(s, z_{1}, \hat{z_{2}}\right)\right) \zeta\left(s, z_{1}\right) \\
& \times \exp \left(-2 \pi \lambda_{1} \int_{z_{1}}^{\infty}\left(1-\exp \left(g\left(s, v, \hat{z_{2}}\right)\right) \zeta(s, v)\right) v \mathrm{~d} v\right)
\end{aligned}
$$

where the proof follows on the same lines as Theorem 8 except that here the field of small cell interferers contains all the holes that are individually carved out from the baseline PPP, thus underestimating the interference from small cells. The final result follows from deconditioning over serving distance $\hat{Z}_{2}$ and then over distance $Z_{1}$ whose distribution is given by (4.2). 


\section{Appendix C}

\section{C.1 Proof of Theorem 10}

The Laplace transform of the aggregate interference power at the primary user is

$$
\begin{aligned}
\mathcal{L}_{I}(s) & =\mathbb{E}\left[\exp \left(-s \sum_{\mathbf{x} \in \Phi_{\mathrm{c}} \cap \mathbf{b}^{c}(0, D)} P_{\mathrm{c}} h_{\mathbf{x}}\left\|\mathbf{x}-\mathbf{x}_{\mathrm{p}}\right\|^{-\alpha}\right)\right] \\
& =\mathbb{E}_{\Phi_{\mathrm{c}}}\left[\prod_{\left.\mathbf{x} \in \Phi_{\mathrm{c}} \cap \mathbf{b}^{c}(0, D)\right)} \mathbb{E}_{h_{\mathbf{x}}}\left[\exp \left(-s P_{\mathrm{c}} h_{\mathbf{x}}\left\|\mathbf{x}-\mathbf{x}_{\mathrm{p}}\right\|^{-\alpha}\right)\right]\right] \\
& =\mathbb{E}_{\Phi_{\mathrm{c}}}\left[\prod_{\left.\mathbf{x} \in \Phi_{\mathrm{c}} \cap \mathbf{b}^{c}(0, D)\right)} \frac{1}{1+s P_{\mathrm{c}}\left\|\mathbf{x}-\mathbf{x}_{\mathrm{p}}\right\|^{-\alpha}}\right] \\
& \stackrel{(\mathrm{a})}{=} \exp \left(-\lambda_{\mathrm{c}} \int_{\mathcal{S}} \frac{1}{1+\frac{\left\|\mathbf{x}-\mathbf{x}_{\mathrm{p}}\right\|^{-\alpha}}{P_{\mathrm{c}} s}} \mathrm{~d} x\right) \\
& \stackrel{(\mathrm{b})}{=} \exp \left(\int_{D-r_{\mathrm{p}}}^{D+r_{\mathrm{p}}} \frac{-2 \pi \lambda(r)}{1+\frac{r^{\alpha}}{P_{\mathrm{c}}}} r \mathrm{~d} r-\int_{D+r_{\mathrm{p}}}^{\infty} \frac{2 \pi \lambda_{\mathrm{c}}}{1+\frac{r^{\alpha}}{P_{\mathrm{c}} s}} r \mathrm{~d} r\right)
\end{aligned}
$$

where $\mathcal{S}$ denotes the interference field, (a) follows from probability generating functional of Poisson distribution [65], and (b) follows from converting 
Cartesian to polar coordinates which transforms the asymmetric exclusion zone into the symmetric one with the non-homogeneous density of $\lambda(r)=$ $\left(1-\frac{\arccos \left(\frac{r^{2}+r_{\mathrm{p}}{ }^{2}-D^{2}}{2 \mathrm{p}_{\mathrm{p}} r}\right)}{\pi}\right) \lambda_{\mathrm{c}}$. 